\title{
ON THE GLOBAL REGULARITY OF 2-D DENSITY PATCH FOR INHOMOGENEOUS INCOMPRESSIBLE VISCOUS FLOW
}

\author{
XIAN LIAO AND PING ZHANG
}

\begin{abstract}
Toward P.-L. Lions' open question in [22] concerning the propagation of regularity for density patch, we establish the global existence of solutions to the 2-D inhomogeneous incompressible Navier-Stokes system with initial density given by $(1-\eta) \mathbf{1}_{\Omega_{0}}+\mathbf{1}_{\Omega_{0}^{c}}$ for some small enough constant $\eta$ and some $W^{k+2, p}$ domain $\Omega_{0}$, and with initial vorticity belonging to $L^{1} \cap L^{p}$ and with appropriate tangential regularities. Furthermore, we prove that the regularity of the domain $\Omega_{0}$ is preserved by time evolution.
\end{abstract}

Keywords: Inhomogeneous incompressible Navier-Stokes equations, density patch, striated distributions, Littlewood-Paley Theory

AMS Subject Classification (2000): 35Q30, 76D03

\section{INTRODUCTION}

We consider the following inhomogeneous incompressible Navier-Stokes equations in two space dimension:

$$
\left\{\begin{array}{l}
\partial_{t} \rho+\operatorname{div}(\rho v)=0, \quad(t, x) \in \mathbb{R}^{+} \times \mathbb{R}^{2}, \\
\partial_{t}(\rho v)+\operatorname{div}(\rho v \otimes v)-\Delta v+\nabla \pi=0 \\
\operatorname{div} v=0 \\
\left.(\rho, v)\right|_{t=0}=\left(\rho_{0}, v_{0}\right)
\end{array}\right.
$$

where $\rho \in \mathbb{R}^{+}, v \in \mathbb{R}^{2}$ and $\pi \in \mathbb{R}$ stand for the density, velocity field and pressure of the fluid, respectively. The above system describes a fluid that is incompressible but has nonconstant density. Basic examples are mixture of incompressible and non reactant flows, flows with complex structure (e.g. blood flow or model of rivers), fluids containing a melted substance, etc.

A lot of recent works have been dedicated to the mathematical study of the above system. Global weak solutions with finite energy have been constructed by J. Simon in [24] (see also the book by P.-L. Lions [22] for the variable viscosity case). In the case of smooth data with no vacuum, the existence of strong unique solutions goes back to the work of O. Ladyzhenskaya and V. Solonnikov in [20]. More recently, R. Danchin [13] established the well-posedness of the above system in the whole space $\mathbb{R}^{d}$ in the so-called critical functional framework for small perturbations of some positive constant density. The basic idea is to use functional spaces (or norms) that have the same scaling invariance as (1.1), namely

$$
(\rho, v, \pi)(t, x) \longmapsto\left(\rho, \lambda v, \lambda^{2} \pi\right)\left(\lambda^{2} t, \lambda x\right), \quad\left(\rho_{0}, v_{0}\right)(x) \longmapsto\left(\rho_{0}, \lambda v_{0}\right)(\lambda x) .
$$

More precisely, in [13], global well-posedness was established assuming that for some small enough constant $c$, one has

$$
\left\|\rho_{0}-1\right\|_{\dot{B}_{2, \infty}^{\frac{d}{2}}\left(\mathbb{R}^{d}\right) \cap L^{\infty}\left(\mathbb{R}^{d}\right)}+\left\|v_{0}\right\|_{\dot{B}_{2,1}^{-1+\frac{d}{2}}\left(\mathbb{R}^{d}\right)} \leq c .
$$

Date: $3 / 17 / 2015$. 
Above $\dot{B}_{p, r}^{\sigma}\left(\mathbb{R}^{d}\right)$ stands for a homogeneous Besov space on $\mathbb{R}^{d}$. This result was extended to more general Besov spaces by H. Abidi in [1], and by H. Abidi and M. Paicu in [2], and to more general smallness condition by the second author and M. Paicu in [23]. The smallness assumption on the initial density was removed recently in $[3,4]$.

Given that in all those works the density has to be at least in the Besov space $\dot{B}_{p, \infty}^{\frac{d}{p}}\left(\mathbb{R}^{d}\right)$, one cannot capture discontinuities across an hypersurface. In effect, the Besov regularity of the characteristic function of a smooth domain is only $\dot{B}_{p, \infty}^{\frac{1}{p}}\left(\mathbb{R}^{d}\right)$. Therefore, those results do not apply to a mixture of two fluids with different densities.

In [14], R. Danchin and P. Mucha proved the global existence and uniqueness of solutions to $(1.1)$ for any data $\left(\rho_{0}, u_{0}\right)$ such that for some $p \in[1,2 d[$ and small enough constant $c$, there holds

$$
\left\|\rho_{0}-1\right\|_{\mathcal{M}\left(\dot{B}_{p, 1}^{-1+\frac{d}{p}}\left(\mathbb{R}^{d}\right)\right)}+\left\|v_{0}\right\|_{\dot{B}_{p, 1}^{-1+\frac{d}{p}}\left(\mathbb{R}^{d}\right)} \leq c .
$$

Above, $\|\cdot\|_{\mathcal{M}\left(\dot{B}_{p, 1}^{-1+\frac{d}{p}}\left(\mathbb{R}^{d}\right)\right)}$ denotes the multiplier norm associated to the Besov space $\dot{B}_{p, 1}^{-1+\frac{d}{p}}\left(\mathbb{R}^{d}\right)$, which turns out to be finite for characteristic functions of $C^{1}$ domains whenever $p>d-1$. Therefore, initial densities with a discontinuity across an interface may be considered (although the jump has to be small owing to (1.2)). In fact, even $\rho_{0}$ is only bounded and bounded away from zero, J. Huang, M. Paicu and the second author in [19] could solve the global existence of solutions to (1.1) in a critical functional framework, and uniqueness was obtained if assuming slightly more regularity for the velocity field. A half space setting of this problem was solved by R. Danchin and the second author lately in [15].

A natural question to ask is whether it is still possible to propagate higher order regularities of the interface of the fluids. Especially, P.-L. Lions proposed the following open question in [22]: suppose the initial density $\rho_{0}=1_{D}$ for some smooth domain $D$, Theorem 2.1 of [22] yields at least one global weak solution $(\rho, v)$ of $(1.1)$ such that for all $t \geq 0, \rho(t)=1_{D(t)}$ for some set $D(t)$ with $\operatorname{vol}(D(t))=\operatorname{vol}(D)$. Then whether or not the regularity of $D$ is preserved by the time evolution. Since this problem is very sophisticated due to the appearance of vacuum, as a first step toward this question, here we aim at solving the global well-posedness of $(1.1)$ with the initial density $\rho_{0}(x)=(1-\eta) \mathbf{1}_{\Omega_{0}}+\mathbf{1}_{\Omega_{0}^{c}}$ for some simply connected $W^{k+2, p}\left(\mathbb{R}^{2}\right)$ domain $\Omega_{0}$ and $\eta$ being sufficiently small.

More precisely, let $\Omega_{0}$ be a simply connected $W^{k+2, p}$, for $\left.k \geq 1, p \in\right] 2,4[$, bounded domain. Let $f_{0} \in W^{k+2, p}\left(\mathbb{R}^{2}\right)$ such that $\partial \Omega_{0}=f_{0}^{-1}(\{0\})$ and $\nabla f_{0}$ does not vanish on $\partial \Omega_{0}$. Then we can parametrize $\partial \Omega_{0}$ as

$$
\gamma_{0}: \mathcal{S}^{1} \mapsto \partial \Omega_{0} \text { via } s \mapsto \gamma_{0}(s) \text { with } \partial_{s} \gamma_{0}(s)=\nabla^{\perp} f_{0}\left(\gamma_{0}(s)\right)
$$

We take the initial density $\rho_{0}$ and the initial vorticity $\omega_{0}$ of (1.1) as follows

$$
\rho_{0}=(1-\eta) \mathbf{1}_{\Omega_{0}}+\mathbf{1}_{\Omega_{0}^{c}}, \quad \text { and } \quad \omega_{0} \in L^{1}\left(\mathbb{R}^{2}\right) \quad \text { with } \quad \partial_{X_{0}}^{\ell} \omega_{0} \in W^{-\frac{\ell}{k} \varepsilon, p}\left(\mathbb{R}^{2}\right)
$$

for some $\varepsilon \in] 0,1\left[, \ell=0, \cdots, k\right.$, and $X_{0}=\nabla^{\perp} f_{0}, \partial_{X_{0}} \omega_{0} \stackrel{\text { def }}{=} X_{0} \cdot \nabla \omega_{0}$.

The main motivation for us to solve the above problem comes from $[8,9,10,11]$. Let us mention that by using the idea of conormal distributions or striated distributions, J.-Y. Chemin $[9,10]$ (see also [6]) proved the global regularities for the vortex patch problem of 2-D incompressible Euler system. One may also check the following papers as extensions of the results established in [9, 10]: P. Gamblin and X. Saint Raymond [16] and the second 
author and Q. Qiu [25] for the 3-D extensions, and R. Danchin [12] and T. Hmidi [17, 18] for the viscous extensions. We mention that the tools in $[8,9,10,11]$ will play an essential role in this paper.

The main result of this paper states as follows:

Theorem 1.1. Let the initial data $\left(\rho_{0}, v_{0}\right)$ be given by (1.4) for $\eta$ sufficiently small. Then (1.1) has a unique global solution $(\rho, v)$ so that $\rho(t, x)=(1-\eta) \mathbf{1}_{\Omega(t)}+\mathbf{1}_{\Omega(t)^{c}}$ for some simply connected $W^{k+2, p}$ domain $\Omega(t)$.

Remark 1.1. For $\Omega_{0}$ given by (1.3), it is easy to observe that $\partial_{X_{0}}^{\ell} \mathbf{1}_{\Omega_{0}}(x)=0$ for any $\ell \in\{1, \cdots, k\}$. Hence in particular, Theorem 1.1 ensures the global well-posedness of (1.1) with initial density $\rho_{0}=(1-\eta) \mathbf{1}_{\Omega_{0}}+\mathbf{1}_{\Omega_{0}^{c}}$ and initial vorticity $\omega_{0}=\mathbf{1}_{\Omega_{0}}(x)$. Furthermore, the geometric structure of $\Omega_{0}$ will be persisted for all time.

Remark 1.2. (1) Compared with the vector field method in $[8,9,10,11]$, here new difficulties arise due to the non-commutative property of the vector field with the viscosity term and the estimate of the pressure function.

(2) Furthermore due to the transport equation of the density function in (1.1), the estimate of the pressure function is more subtle than that in $[12,17,18]$.

(3) Technically since we shall use the maximal regularity estimate for evolutionary Stokes operator, we shall use striated distributions in the framework of the Sobolev space, $W^{k, p}$, which is of Triebel-Lizorkin type space, yet the striated distributions in the previous papers are in the framework of Besov type spaces.

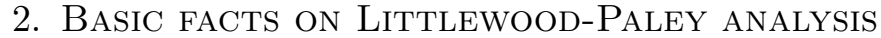

For the convenience of the reader, we recall some basic facts on Littlewood-Paley theory, and one may check Chapter 2 of [5] for more details. Let us briefly explain how it may be built in the case $x \in \mathbb{R}^{d}$ (see e.g. [5]). Let $\chi, \varphi$ be smooth functions supported in the ball $\mathcal{B} \stackrel{\text { def }}{=}\left\{\xi \in \mathbb{R}^{d},|\xi| \leq \frac{4}{3}\right\}$ and the $\operatorname{ring} \mathcal{C} \stackrel{\text { def }}{=}\left\{\xi \in \mathbb{R}^{d}, \frac{3}{4} \leq|\xi| \leq \frac{8}{3}\right\}$ respectively, such that

$$
\sum_{j \in \mathbb{Z}} \varphi\left(2^{-j} \xi\right)=1 \quad \text { for } \quad \xi \neq 0 \quad \text { and } \quad \chi(\xi)+\sum_{j \geq 0} \varphi\left(2^{-j} \xi\right)=1 \quad \text { for } \quad \xi \in \mathbb{R}^{d} .
$$

Then for $u \in \mathcal{S}^{\prime}\left(\mathbb{R}^{d}\right)$, we set

$$
\begin{aligned}
& \forall j \in \mathbb{Z}, \quad \dot{\Delta}_{j} u=\varphi\left(2^{-j} D\right) u \quad \text { and } \quad \dot{S}_{j} u=\chi\left(2^{-j} D\right) u, \\
& \forall j \in \mathbb{N}, \quad \Delta_{j} u=\varphi\left(2^{-j} D\right) u, \quad \Delta_{-1} u=\chi(D) u \quad \text { and } \quad S_{j} u=\sum_{j^{\prime} \leq j-1} \Delta_{j^{\prime}} u .
\end{aligned}
$$

The dyadic blocks satisfies the property of almost orthogonality:

$$
\dot{\Delta}_{k} \dot{\Delta}_{j} u \equiv 0 \quad \text { if } \quad|k-j| \geq 2 \quad \text { and } \quad \dot{\Delta}_{k}\left(\dot{S}_{j-1} u \dot{\Delta}_{j} u\right) \equiv 0 \quad \text { if } \quad|k-j| \geq 5 .
$$

We first recall the definition of homogeneous Besov spaces.

Definition 2.1. Let $(p, r) \in[1,+\infty]^{2}, s \in \mathbb{R}$ and $u \in \mathcal{S}_{h}^{\prime}\left(\mathbb{R}^{d}\right)$, which means that $u$ is in $\mathcal{S}^{\prime}\left(\mathbb{R}^{d}\right)$ and satisfies $\lim _{j \rightarrow-\infty}\left\|\dot{S}_{j} u\right\|_{L^{\infty}}=0$. We set

$$
\|u\|_{\dot{B}_{p, r}^{s}} \stackrel{\text { def }}{=}\left\|\left(2^{j s}\left\|\dot{\Delta}_{j} u\right\|_{L^{p}}\right)\right\|_{\ell^{r}} .
$$

- For $s<\frac{d}{p}$ (or $s=\frac{d}{p}$ if $r=1$ ), we define $\dot{B}_{p, r}^{s}\left(\mathbb{R}^{d}\right) \stackrel{\text { def }}{=}\left\{u \in \mathcal{S}^{\prime}\left(\mathbb{R}^{d}\right) \mid\|u\|_{\dot{B}_{p, r}^{s}}<\infty\right\}$. 
- If $k \in \mathbb{N}$ and $\frac{d}{p}+k \leq s<\frac{d}{p}+k+1$ (or $s=\frac{d}{p}+k+1$ if $r=1$ ), then $\dot{B}_{p, r}^{s}\left(\mathbb{R}^{d}\right.$ ) is defined as the subset of distributions $u \in \mathcal{S}^{\prime}\left(\mathbb{R}^{d}\right)$ such that $\partial^{\beta} u \in \dot{B}_{p, r}^{s-k}\left(\mathbb{R}^{d}\right)$ whenever $|\beta|=k$.

We also need the following version of Bernstein Lemma:

Lemma 2.1. Let $\mathcal{B}$ be a ball and $\mathcal{C}$ a ring of $\mathbb{R}^{d}$. A constant $C$ exists so that for any positive real number $\lambda$, any non negative integer $k$, any smooth homogeneous function $\sigma$ of degree $m$, and any couple of real numbers $(a, b)$ with $b \geq a \geq 1$, there hold

$$
\begin{aligned}
& \text { Supp } \hat{u} \subset \lambda \mathcal{B} \Rightarrow \sup _{|\alpha|=k}\left\|\partial^{\alpha} u\right\|_{L^{b}} \leq C^{k+1} \lambda^{k+d\left(\frac{1}{a}-\frac{1}{b}\right)}\|u\|_{L^{a}}, \\
& \text { Supp } \hat{u} \subset \lambda \mathcal{C} \Rightarrow C^{-1-k} \lambda^{k}\|u\|_{L^{a}} \leq \sup _{|\alpha|=k}\left\|\partial^{\alpha} u\right\|_{L^{a}} \leq C^{1+k} \lambda^{k}\|u\|_{L^{a}}, \\
& \text { Supp } \hat{u} \subset \lambda \mathcal{C} \Rightarrow\|\sigma(D) u\|_{L^{b}} \leq C_{\sigma, m} \lambda^{m+d\left(\frac{1}{a}-\frac{1}{b}\right)}\|u\|_{L^{a}} \\
& \text { Supp } \hat{u} \subset \lambda \mathcal{C} \Rightarrow\left\|e^{t \Delta} u\right\|_{L^{a}} \leq C e^{-c \lambda^{2} t}\|u\|_{L^{a}} .
\end{aligned}
$$

Finally let us recall Bony's decomposition in the inhomogeneous context from [7]:

$$
u v=T_{u} v+T_{v} u+R(u, v)
$$

where

$$
T_{u} v \stackrel{\text { def }}{=} \sum_{j \in \mathbb{Z}} S_{j-1} u \Delta_{j} v, \quad R(u, v) \stackrel{\text { def }}{=} \sum_{j \in \mathbb{Z}} \Delta_{j} u \widetilde{\Delta}_{j} v \quad \text { with } \quad \widetilde{\Delta}_{j} v \stackrel{\text { def }}{=} \sum_{\left|j^{\prime}-j\right| \leq 1} \Delta_{j^{\prime}} v .
$$

We sometime will also use Bony's decomposition in homogeneous context.

\section{IDEAS OF THE PROOF AND STRUCTURE OF THE PAPER}

Because we shall consider only perturbations of the reference density 1 , it is natural to set $a \stackrel{\text { def }}{=} 1 / \rho-1$ so that System (1.1) translates into

$$
\left\{\begin{array}{l}
\partial_{t} a+v \cdot \nabla a=0 \quad(t, x) \in \mathbb{R}^{+} \times \mathbb{R}^{2}, \\
\partial_{t} v+v \cdot \nabla v+(1+a)(\nabla \pi-\Delta v)=0 \\
\operatorname{div} v=0 \\
\left.(a, v)\right|_{t=0}=\left(a_{0}, v_{0}\right) .
\end{array}\right.
$$

Before proceeding, let us recall the striated distribution spaces with respect to the vector field $X$ from $[8,9]$ :

$$
C_{\delta}^{\sigma}(X, k) \stackrel{\text { def }}{=}\left\{f \in C^{\sigma}\left(\mathbb{R}^{2}\right) \mid\|f\|_{\delta, X}^{\sigma, k} \stackrel{\text { def }}{=} \sum_{\ell=0}^{k}\left\|\left(T_{X \cdot \nabla}\right)^{\ell} f\right\|_{C^{\sigma-\ell \delta}}<+\infty\right\}, \quad \sigma \in \mathbb{R}^{*} \backslash\{1\},
$$

where $T_{a} b$ denotes the para-product of $b$ by $a$ (see (2.4)). And we call a vector field $X$ is $\delta, k$-regular if

$$
\left.\|X\|_{\delta, X}^{1-\delta, k-1}<\infty, \quad \text { with } \delta \in\right] 0,1[\text { and } k \delta<1 .
$$

However as in $[15,19]$, the functional framework in this work for solving (3.1) is motivated by classical maximal regularity estimates for the evolutionary Stokes system, so that it is more natural to introduce the striated distribution space in the framework of Sobolev space $W^{s, p}$

$$
W_{\delta}^{s, p}(X, k) \stackrel{\text { def }}{=}\left\{\left.f \in W^{s, p}\left(\mathbb{R}^{2}\right)|| f\right|_{\delta, X} ^{s, p, k} \stackrel{\text { def }}{=} \sum_{\ell=0}^{k}\left\|\left(T_{X \cdot \nabla}\right)^{\ell} f\right\|_{W^{s-\ell \delta, p}}<+\infty\right\} .
$$


Corresponding to (3.4), we also define

$$
\mathcal{W}_{\delta}^{s, p}(X, k) \stackrel{\text { def }}{=}\left\{f \in W^{s, p}\left(\mathbb{R}^{2}\right)|| f\left\|_{\delta, X}^{s, p, k} \stackrel{\text { def }}{=} \sum_{\ell=0}^{k}\right\| \partial_{X}^{\ell} f \|_{W^{s-\ell \delta, p}}<+\infty\right\} .
$$

Here and in all that follows, we always denote $\partial_{X} f=X \cdot \nabla f$. We shall prove in Lemma 7.2 below that the function spaces $W_{\delta}^{s, p}(X, k)$ and $\mathcal{W}_{\delta}^{s, p}(X, k)$ are the same provided that the vector field $X$ is $\delta, k$-regular, $s \in] k \delta, 1[$ or $s \in] k \delta-1,1[$ if $\operatorname{div} X=0$.

We shall first prove the following more general result:

Theorem 3.1. Let $p \in] 2,4\left[\right.$, let $X_{0}=\left(X_{0}^{1}, X_{0}^{2}\right)$ and $\left(a_{0}, v_{0}\right)$ satisfy $\operatorname{div} X_{0}=0, \partial_{X_{0}}^{\ell-1} X_{0} \in$ $W^{2, p}, \partial_{X_{0}}^{\ell} a_{0} \in L^{\infty}$ for $\ell \in\{1, \cdots, k\}$ and $a_{0} \in L^{2}, v_{0} \in \mathcal{W}_{\varepsilon / k}^{1, p}\left(X_{0}, k\right)$ for some $\left.\varepsilon \in\right] 0,1[$. Then there exists a positive constant $c_{0}$ so that whenever $\left\|a_{0}\right\|_{L^{\infty}} \leq c_{0}$, there exists a divergence free vector field $X(t)$ which satisfies

$$
\left\{\begin{array}{l}
\partial_{t} X+v \cdot X=X \cdot \nabla v \\
X(0, x)=X_{0}(x)
\end{array}\right.
$$

$\partial_{X}^{\ell-1} X \in L_{\text {loc }}^{\infty}\left(\mathbb{R}^{+} ; W^{2, p}\right)$ for $\ell \in\{1, \cdots, k\}$, and (3.1) has a unique global solution $(a, v)$ with

$$
\begin{aligned}
\left\|\left(\partial_{t} \partial_{X}^{\ell} v, \Delta \partial_{X}^{\ell} v, \nabla \partial_{X}^{\ell} \pi\right)\right\|_{L_{t}^{r \ell}\left(L^{p}\right)}+\left\|\partial_{X}^{\ell} a\right\|_{L_{t}^{\infty}\left(L^{\infty}\right)}+\left\|\nabla \partial_{X}^{\ell} v\right\|_{L_{t}^{r_{\ell}}\left(L^{\infty}\right) \cap L_{t}^{s \ell}\left(L^{p}\right)} \\
+\left\|\partial_{X}^{\ell} v\right\|_{L_{t}^{s \ell}\left(L^{\infty}\right)}+\left\|\partial_{t} \partial_{X}^{\ell-1} X\right\|_{L_{t}^{s \ell}\left(W^{1, p}\right)}+\left\|\partial_{X}^{\ell-1} X\right\|_{L_{t}^{\infty}\left(W^{2, p}\right)} \leq \mathcal{H}_{\ell}(t),
\end{aligned}
$$

for $\ell \in\{0, \cdots, k\}$ (with the convention: $\partial_{X}^{-1} X \stackrel{\text { def }}{=} 0$ ). Here and in all that follows, we always denote $r_{\ell}, s_{\ell}$ to be any numbers with $\left.r_{\ell} \in\right] 1, \frac{2 k}{k+\ell \varepsilon}\left[\right.$ and $\left.s_{\ell} \in\right] 2, \frac{2 k}{\ell \varepsilon}\left[\right.$, and $\mathcal{H}_{\ell}(t), \ell=0, \cdots, k$, to be positive increasing functions of $t$ which depend on the initial data, $r_{\ell}, s_{\ell}$, and which may change from line to line.

As an application of the above theorem, we consider the propagation of the boundary regularity for the 2-D density patch's problem of (1.1).

Theorem 3.2. Let the initial data $\left(\rho_{0}, v_{0}\right)$ be given by (1.4) for $\eta$ sufficiently small. Then (1.1) has a unique global solution $(\rho, v)$ so that $\rho(t, x)=(1-\eta) \mathbf{1}_{\Omega(t)}+\mathbf{1}_{\Omega(t)^{c}}$ for some simply connected $W^{k+2, p}$ domain $\Omega(t)$, which is determined by the level surface of $f \in$ $L_{\text {loc }}^{\infty}\left(\mathbb{R}^{+} ; W^{k+2, p}\right)$ so that with $X \stackrel{\text { def }}{=} \nabla^{\perp} f$, there holds (3.7).

It is easy to observe that Theorem 1.1 is a direct consequence of Theorem 3.2.

As an illustration to the main idea of the proof to Theorem 3.2, we first sketch the proof of Theorem 3.2 for the case $k=1$ by assuming Theorem 3.1.

- Sketch of the proof of Theorem 3.2 for $k=1$. Let $\psi(t, \cdot)$ be the flow associated with the velocity vector field $v$, that is

$$
\left\{\begin{array}{l}
\frac{d}{d t} \psi(t, x)=v(t, \psi(t, x)) \\
\psi(0, x)=x
\end{array}\right.
$$

Then by virtue of Theorem 3.1, one has

$$
\psi(t, \cdot)-\operatorname{Id} \in L_{\mathrm{loc}}^{\infty}\left(\mathbb{R}^{+} ; W^{2, p}\right),
$$

and hence $\Omega(t) \stackrel{\text { def }}{=} \psi\left(t, \Omega_{0}\right)$ is a $W^{2, p}$ domain. Moreover, it follows from the transport equation of (1.1) that

$$
\rho(t, x)=(1-\eta) \mathbf{1}_{\Omega(t)}+\mathbf{1}_{\Omega(t)^{c}} .
$$


Next we are going to prove that $\Omega(t)$ is of class $W^{3, p}$. As a matter of fact, let $\partial \Omega(t)$ be the level surface of $f(t, \cdot)$. Then $f$ solves

$$
\left\{\begin{array}{l}
\partial_{t} f+v \cdot \nabla f=0 \\
f(0, x)=f_{0}(x)
\end{array}\right.
$$

which implies that $X(t, x) \stackrel{\text { def }}{=} \nabla^{\perp} f(t, x)$ solves (3.6). Thus it follows from (3.6) and (3.8) that

$$
X(t, \psi(t, x))=X_{0}(x) \frac{\partial \psi(t, x)}{\partial x} .
$$

Moreover, due to $\operatorname{div} X_{0}=0=\operatorname{div} v$, one has

$$
\partial_{t} \operatorname{div} X+v \cdot \operatorname{div} X=0 \quad \text { with } \quad \operatorname{div} X_{0}=0
$$

Hence $\operatorname{div} X(t)=0$, and according to Theorem 3.1, we have

$$
X \in L_{\mathrm{loc}}^{\infty}\left(\mathbb{R}^{+} ; W^{2, p}\right) .
$$

It is easy to observe that $X \in L_{\mathrm{loc}}^{\infty}\left(L^{p}\right)$, and

$$
\begin{aligned}
\partial_{j} \partial_{j}\left(X^{k}(t, \psi(t, x))\right)= & \partial_{i} \partial_{j} \psi(t, x) \cdot \nabla X^{k}(t, \psi(t, x)) \\
& +\left(\partial_{i} \psi \otimes \partial_{j} \psi\right)(t, x): \nabla^{2} X^{k}(t, \psi(t, x)) \in L_{\mathrm{loc}}^{\infty}\left(\mathbb{R}^{+} ; L^{p}\right) .
\end{aligned}
$$

Therefore we deduce from (3.10) that

$$
\partial_{s}\left(\psi\left(t, \gamma_{0}(s)\right)\right)=X_{0}\left(\gamma_{0}(s)\right) \frac{\partial \psi\left(t, \gamma_{0}(s)\right)}{\partial x}=X\left(t, \psi\left(t, \gamma_{0}(s)\right)\right) \in L_{\mathrm{loc}}^{\infty}\left(\mathbb{R}^{+} ; W^{2, p}\right),
$$

that is, $\Omega(t)$ is of class $W^{3, p}$.

Let us now present the proof of Theorem 3.2 by assuming Theorem 3.1.

Proof of Theorem 3.2. In view of (1.4), we observe that $X_{0} \in W^{k+1, p}, k \geq 1, \operatorname{div} X_{0}=0$, and moreover

$$
\partial_{X_{0}}^{\ell} a_{0} \equiv 0, \quad \partial_{X_{0}}^{\ell-1} X_{0} \in W^{k+2-\ell, p} \hookrightarrow W^{2, p}, \quad \ell=1, \cdots, k .
$$

Since $p>2$ and $\nabla v_{0}=\nabla \nabla^{\perp} \Delta^{-1} \omega_{0}$, for any integer $N$, it follows from Lemma 2.1 that

$$
\begin{aligned}
\left\|v_{0}\right\|_{L^{p}} & \lesssim \sum_{j \leq N} 2^{j\left(1-\frac{2}{p}\right)}\left\|\dot{\Delta}_{j} \nabla v_{0}\right\|_{L^{1}}+\sum_{j>N} 2^{-j}\left\|\nabla \dot{\Delta}_{j} v_{0}\right\|_{L^{p}} \\
& \lesssim 2^{N\left(1-\frac{2}{p}\right)}\left\|\omega_{0}\right\|_{L^{1}}+2^{-N}\left\|\omega_{0}\right\|_{L^{p}} .
\end{aligned}
$$

Choosing the best integer $N$ in the above inequality so that

$$
2^{N} \sim\left(\frac{\left\|\omega_{0}\right\|_{L^{p}}}{\left\|\omega_{0}\right\|_{L^{1}}}\right)^{\frac{p}{2(p-1)}}
$$

leads to

$$
\left\|v_{0}\right\|_{L^{p}} \leq C\left\|\omega_{0}\right\|_{L^{1}}^{\frac{p}{2(p-1)}}\left\|\omega_{0}\right\|_{L^{p}}^{\frac{p-2}{2(p-1)}}
$$

As $\nabla v_{0}=\nabla \nabla^{\perp} \Delta^{-1} \omega_{0} \in L^{p}$, we achieve

$$
\left\|v_{0}\right\|_{W^{1, p}} \leq C\left\|\omega_{0}\right\|_{L^{1} \cap L^{p}} .
$$

Moreover, we claim the following striated regularity for $v_{0}$

$$
\left.\left|v_{0}\right|_{\varepsilon / k, X_{0}}^{1, p, k} \leq C\left|\omega_{0}\right|_{\varepsilon / k, X_{0}}^{0, p, k} \quad \text { for any } \quad \varepsilon \in\right] 0,1[
$$


which will be proved in Section 7.

Thanks to (3.13) and (3.15), we deduce from Theorem 3.1 that with initial data $\left(a_{0}, v_{0}\right)$ given by (1.4), (3.1) has a unique solution $(a, v)$ satisfying (3.7). Furthermore, due to $X_{0}=$ $\nabla^{\perp} f_{0}$, it follows from a similar derivation of (3.12) that

$$
\partial_{s}^{k}\left(\psi\left(t, \gamma_{0}(s)\right)\right)=\partial_{s}^{k-1}\left(\left(\partial_{X_{0}} \psi\right)\left(t, \gamma_{0}(s)\right)\right)=\cdots=\left(\partial_{X_{0}}^{k} \psi\right)\left(t, \gamma_{0}(s)\right), \quad \forall k \geq 1 .
$$

Hence in order to show that $\left.\partial \Omega(t)=\psi\left(t, \gamma_{0}\left(\partial \Omega_{0}\right)\right)\right) \in W^{k+2, p}$, it suffices to prove that $\partial_{X_{0}}^{k} \psi \in L_{\mathrm{loc}}^{\infty}\left(W^{2, p}\right)$, which is equivalent to show that $\partial_{X}^{k-1} X \in L_{\mathrm{loc}}^{\infty}\left(W^{2, p}\right)$. Indeed, it follows from (3.8) and (3.6) that

$$
X(t, x)=\left(\partial_{X_{0}} \psi\right)\left(t, \psi^{-1}(t, x)\right)
$$

and hence

$$
\begin{aligned}
\left(\partial_{X} X\right)(t, x) & =\left(\partial_{X_{0}} \psi\right)\left(t, \psi^{-1}(t, x)\right) \cdot \nabla \psi^{-1}(t, x) \cdot\left(\nabla \partial_{X_{0}} \psi\right)\left(t, \psi^{-1}(t, x)\right) \\
& =X_{0}\left(t, \psi^{-1}(t, x)\right) \cdot(\nabla \psi)\left(t, \psi^{-1}(t, x)\right) \cdot \nabla \psi^{-1}(t, x) \cdot\left(\nabla \partial_{X_{0}} \psi\right)\left(t, \psi^{-1}(t, x)\right) \\
& =X_{0}\left(t, \psi^{-1}(t, x)\right) \cdot\left(\nabla \partial_{X_{0}} \psi\right)\left(t, \psi^{-1}(t, x)\right) \\
& =\left(\partial_{X_{0}}^{2} \psi\right)\left(t, \psi^{-1}(t, x)\right) .
\end{aligned}
$$

The same arguments yield that

$$
\left(\partial_{X}^{k-1} X\right)(t, x)=\left(\partial_{X_{0}}^{k} \psi\right)\left(t, \psi^{-1}(t, x)\right), \quad \forall k \geq 1,
$$

which implies $\partial_{X_{0}}^{k} \psi \in L_{\text {loc }}^{\infty}\left(W^{2, p}\right)$ if $\partial_{X}^{k-1} X \in L_{\text {loc }}^{\infty}\left(W^{2, p}\right)$. And hence by virtue of (3.7), we conclude that $\partial \Omega(t) \in W^{k+2, p}$. This completes the proof of Theorem 3.2.

The structure of the paper is organized as follows:

Section 4, Section 5 and Section 6 are devoted to the proof of Theorem 3.1: Section 4 is devoted to the proof of the Estimate (3.7) for $\ell=0$; in Section 5 we prove the Estimate (3.7) for the case $\ell=1$; and in Section 6 we first derive the Estimate (3.7) for the general case $\ell=2, \cdots, k$, and then Theorem 3.1 will be proved at the end.

We concentrate on the proof of (3.15) in Section 7 and the appendix will be devoted to the proofs of Lemma 7.1 and Lemma 7.2.

Let us complete this section with the notations we are going to use in this context.

Notations: $C^{\sigma}$ denotes the classical Hölder space, $W^{s, p}$ stands for the Sobolev space with norm given by $\|f\|_{W^{s, p}} \stackrel{\text { def }}{=}\left\|(I d-\Delta)^{\frac{s}{2}} f\right\|_{L^{p}}$. We denote $[A ; B]=A B-B A$ the commutator of $A$ and $B$. For $X$ a Banach space and $I$ an interval of $\mathbb{R}$, we denote by $L^{q}(I ; X)$ the set of measurable functions on $I$ with values in $X$, such that $t \longmapsto\|f(t)\|_{X}$ belongs to $L^{q}(I)$. For $a \lesssim b$, we mean that there is a uniform constant $C$, which may be different on different lines, such that $a \leq C b$.

\section{The PRELIMINARY ESTIMATES}

In this section, we shall prove the Estimate (3.7) for $\ell=0$, which will be based on the energy estimate of the System (1.1).

Lemma 4.1. Let $p \in] 2,4[, r \in] 1,2[, s \in] 2, \infty]$ and $q \in] \frac{2 p}{p+1}, 2 p\left[\right.$. Let $v_{0} \in W^{1, p}$ and $v_{L}(t) \stackrel{\text { def }}{=}$ $e^{t \Delta} v_{0}$. Then there holds

$$
\left\|\Delta v_{L}\right\|_{L_{t}^{r}\left(L^{p}\right)}+\left\|\nabla v_{L}\right\|_{L_{t}^{s}\left(L^{p}\right)}+\left\|\nabla v_{L}\right\|_{L_{t}^{q}\left(L^{2 p}\right)} \leq C\left\|v_{0}\right\|_{W^{1, p}}
$$


Proof. Since $p \in] 2,4$, by virtue of Theorem 2.41 of [5], we have $v_{0} \in W^{1, p} \hookrightarrow \dot{B}_{p, p}^{0} \cap \dot{B}_{p, p}^{1}$, where $\dot{B}_{p, q}^{\sigma}$ denotes the homogeneous Besov space (see Definition 2.1). Hence according to Theorem 2.34 of [5] (the definition of Besov spaces with negative indices through heat kernel) that

$$
\begin{aligned}
& \left\|t^{\frac{1}{2}-\frac{1}{p}} \Delta e^{t \Delta} v_{0}\right\|_{L_{t}^{p}\left(L^{p}\right)} \leq C\left\|\Delta v_{0}\right\|_{\dot{B}_{p, p}^{-1}} \leq C\left\|v_{0}\right\|_{W^{1, p}}, \\
& \left\|t^{1-\frac{1}{p}} \Delta e^{t \Delta} v_{0}\right\|_{L_{t}^{p}\left(L^{p}\right)} \leq C\left\|\Delta v_{0}\right\|_{\dot{B}_{p, p}^{-2}} \leq C\left\|v_{0}\right\|_{W^{1, p}},
\end{aligned}
$$

so that for any $r \in] 1,2[$ and $t>0$, one has

$$
\begin{aligned}
\left\|\Delta e^{t \Delta} v_{0}\right\|_{L_{t}^{r}\left(L^{p}\right)} \leq & \left\|t^{\frac{1}{2}-\frac{1}{p}} \Delta e^{t \Delta} v_{0}\right\|_{L^{p}(] 0, t \wedge 1\left[\times \mathbb{R}^{2}\right)}\left\|t^{-\left(\frac{1}{2}-\frac{1}{p}\right)}\right\|_{L^{\bar{r}}(] 0, t \wedge 1[)} \\
& +\left\|t^{1-\frac{1}{p}} \Delta e^{t \Delta} v_{0}\right\|_{L^{p}(] t \wedge 1, t\left[\times \mathbb{R}^{2}\right)}\left\|t^{-\left(1-\frac{1}{p}\right)}\right\|_{L^{\bar{r}}(] t \wedge 1, t[)} \\
\leq & C\left\|v_{0}\right\|_{W^{1, p}},
\end{aligned}
$$

where $\bar{r}=\frac{p r}{p-r}$, and we used the fact that $\bar{r}\left(\frac{1}{2}-\frac{1}{p}\right)<1, \bar{r}\left(1-\frac{1}{p}\right)>1$ for any $\left.r \in\right] 1,2[$.

Along the same line, one has

$$
\left\|t^{\frac{1}{2}-\frac{1}{p}} \nabla e^{t \Delta} v_{0}\right\|_{L_{t}^{p}\left(L^{p}\right)} \leq C\left\|\nabla v_{0}\right\|_{\dot{B}_{p, p}^{-1}} \leq C\left\|v_{0}\right\|_{L^{p}}
$$

so that we have

$$
\begin{aligned}
\left\|\nabla e^{t \Delta} v_{0}\right\|_{L_{t}^{s}\left(L^{p}\right)} \leq & \left\|t^{\frac{1}{2}-\frac{1}{p}} \nabla e^{t \Delta} v_{0}\right\|_{L^{p}(] t \wedge 1, t\left[\times \mathbb{R}^{2}\right)}\left\|t^{-\left(\frac{1}{2}-\frac{1}{p}\right)}\right\|_{L^{\bar{s}}(] t \wedge 1, t[)} \\
& +\left\|\nabla e^{t \Delta} v_{0}\right\|_{L^{\infty}(] 0, t \wedge 1\left[; L^{p}\right)} \leq C\left(\left\|v_{0}\right\|_{L^{p}}+\left\|\nabla v_{0}\right\|_{L^{p}}\right),
\end{aligned}
$$

where $\bar{s}=\frac{p s}{p-s}$ and $\bar{s}\left(\frac{1}{2}-\frac{1}{p}\right)>1$ for any $\left.s \in\right] 2, p\left[\right.$. This together with the fact: $\left\|\nabla e^{t \Delta} v_{0}\right\|_{L^{\infty}\left(\mathbb{R}^{+} ; L^{p}\right)} \lesssim$ $\left\|\nabla v_{0}\right\|_{L^{p}}$, ensures that

$$
\left.\left.\left\|\nabla e^{t \Delta} v_{0}\right\|_{L_{t}^{s}\left(L^{p}\right)} \leq C\left\|v_{0}\right\|_{W^{1, p}} \quad \forall s \in\right] 2, \infty\right] .
$$

Finally it follows from Lemma 2.1 that $\nabla v_{0} \in \dot{B}_{2 p, p}^{-\frac{1}{p}} \cap \dot{B}_{2 p, p}^{-1-\frac{1}{p}} \hookrightarrow \dot{B}_{2 p, 2 p}^{-\frac{1}{p}} \cap \dot{B}_{2 p, 2 p}^{-1-\frac{1}{p}}$. Then we deduce from Theorem 2.34 of [5] that

$$
\begin{aligned}
\left\|\nabla e^{t \Delta} v_{0}\right\|_{L_{t}^{q}\left(L^{2 p}\right)} & \leq\left\|\nabla e^{t \Delta} v_{0}\right\|_{L^{2 p}(] 0, t \wedge 1\left[; L^{2 p}\right)}+\left\|t^{\frac{1}{2}} \nabla e^{t \Delta} v_{0}\right\|_{L^{2 p}(] t \wedge 1, t\left[L^{2 p}\right)}\left\|t^{-\frac{1}{2}}\right\|_{L^{\bar{q}}(] t \wedge 1, t[)} \\
& \leq C\left(\left\|\nabla v_{0}\right\|_{\dot{B}_{2 p, 2 p}^{-\frac{1}{p}}}+\left\|\nabla v_{0}\right\|_{\dot{B}_{2 p, 2 p}^{-1-\frac{1}{p}}}\right) \leq C\left\|v_{0}\right\|_{W^{1, p}},
\end{aligned}
$$

$\bar{q}=\frac{2 p q}{2 p-q}$, and we used the fact that $\frac{\bar{q}}{2}>1$ for any $q$ satisfying $\left.q \in\right] \frac{2 p}{p+1}, 2 p[$.

By summing up (4.2), (4.3) and (4.4), we achieve (4.1).

Proposition 4.1. Let $p \in] 2,4\left[, v_{0} \in W^{1, p}\right.$, and $\rho_{0}$ satisfy

$$
\rho_{0}-1 \in L^{2}, \quad m \leq \rho_{0} \leq M
$$

Let $(\rho, v)$ be a smooth enough solution of $(1.1)$ on $[0, T]$. Then there exists a positive constant, $C$, which depends on $m, M,\left\|\rho_{0}-1\right\|_{L^{2}}$ and $\left\|v_{0}\right\|_{W^{1, p}}$ so that

$$
\left\|\left(v-v_{L}\right)(t)\right\|_{L^{2}}^{2}+\left\|\nabla\left(v-v_{L}\right)\right\|_{L_{t}^{2}\left(L^{2}\right)}^{2} \leq C\left(1+t^{2}\right)^{\left(1-\frac{2}{p}\right)} \exp \left(C(1+t)^{\frac{1}{2}}\right) \stackrel{\text { def }}{=} \mathrm{h}(t)
$$

for any $t \leq T$. 
Proof. We first deduce from (1.1) and (4.5) that

$$
\|\rho(t, \cdot)-1\|_{L^{2}}=\left\|\rho_{0}-1\right\|_{L^{2}}, \quad m \leq \rho(t, x) \leq M .
$$

Let us set $\bar{v}(t) \stackrel{\text { def }}{=} v(t)-v_{L}(t)$. Then in view of (1.1), we write

$$
\begin{aligned}
\rho \partial_{t} \bar{v}+\rho v \cdot \nabla \bar{v}-\Delta \bar{v}+\nabla \pi & =-\rho \partial_{t} v_{L}-\Delta v_{L}-\rho v \cdot \nabla v_{L} \\
& =-(\rho-1) \partial_{t} v_{L}-\rho v \cdot \nabla v_{L}
\end{aligned}
$$

Taking $L^{2}$ inner product of the above equation with $\bar{v}$ and using the transport equation of (1.1), we get

$$
\frac{1}{2} \frac{d}{d t}\|\sqrt{\rho} \bar{v}(t)\|_{L^{2}}^{2}+\|\nabla \bar{v}(t)\|_{L^{2}}^{2}=-\left((\rho-1) \partial_{t} v_{L} \mid \bar{v}\right)_{L^{2}}-\left(\rho v \cdot \nabla v_{L} \mid \bar{v}\right)_{L^{2}} .
$$

By applying the 2-D interpolation inequality that

$$
\left.\|f\|_{L^{q}} \leq C\|f\|_{L^{2}}^{\frac{2}{q}}\|\nabla f\|_{L^{2}}^{1-\frac{2}{q}} \text { for } \forall q \in\right] 2, \infty[,
$$

and Young's inequality, we obtain

$$
\begin{aligned}
\left|\left((\rho-1) \partial_{t} v_{L} \mid \bar{v}\right)_{L^{2}}\right| & \leq\|\rho-1\|_{L^{2}}\left\|\Delta v_{L}\right\|_{L^{p}}\|\bar{v}\|_{L^{\frac{2 p}{p-2}}} \\
\leq & C\left(\left\|\rho_{0}-1\right\|_{L^{2}}^{2}\left\|\Delta v_{L}\right\|_{L^{p}}^{2-\frac{r(p-2)}{p}}\right)^{\frac{1}{2}} \\
& \quad \times\left(\left\|\Delta v_{L}\right\|_{L^{p}}^{r}\|\bar{v}\|_{L^{2}}^{2}\right)^{\frac{p-2}{2 p}}\left(\|\nabla \bar{v}\|_{L^{2}}^{2}\right)^{\frac{1}{p}} \\
& \leq \frac{1}{4}\|\nabla \bar{v}\|_{L^{2}}^{2}+C\left(\left\|\rho_{0}-1\right\|_{L^{2}}^{2}\left\|\Delta v_{L}\right\|_{L^{p}}^{2-\frac{r(p-2)}{p}}+\left\|\Delta v_{L}\right\|_{L^{p}}^{r}\|\bar{v}\|_{L^{2}}^{2}\right),
\end{aligned}
$$

for any $r \in] 1,2[$.

Whereas it is easy to observe that

$$
\begin{aligned}
\left|\left(\rho \bar{v} \cdot \nabla v_{L} \mid \bar{v}\right)_{L^{2}}\right| & \leq C\left\|\nabla v_{L}\right\|_{L^{p}}\|\bar{v}\|_{L^{\frac{2 p}{p-1}}}^{2} \\
& \leq C\left\|\nabla v_{L}\right\|_{L^{p}}\|\bar{v}\|_{L^{2}}^{2\left(1-\frac{1}{p}\right)}\|\nabla \bar{v}\|_{L^{2}}^{\frac{2}{p}} \\
& \leq \frac{1}{4}\|\nabla \bar{v}\|_{L^{2}}^{2}+C\left\|\nabla v_{L}\right\|_{L^{p}}^{\frac{p}{p-1}}\|\bar{v}\|_{L^{2}}^{2} .
\end{aligned}
$$

In order the deal with the last term in (4.9), we need to use the claim that

$$
\left.\left\|v_{L} \otimes v_{L}\right\|_{L_{t}^{\frac{p}{2}}\left(\dot{B}_{2, \frac{p}{2}}^{1}\right)} \leq C\left\|v_{0}\right\|_{L^{p}}^{2} \quad \text { for } \forall p \in\right] 2,4[\text {. }
$$

We admit this inequality for the time being and continue to handle the last term in (4.9). Since $p \in] 2,4[$, we have

$$
\begin{aligned}
\left|\left(\rho v_{L} \cdot \nabla v_{L} \mid \bar{v}\right)_{L^{2}}\right| & \leq\left\|v_{L} \otimes v_{L}\right\|_{\dot{H}^{1}}\|\bar{v}\|_{L^{2}} \\
& \leq C\left(\left\|v_{L} \otimes v_{L}\right\|_{\dot{B}_{2, \frac{p}{2}}^{1}}^{\frac{p}{2}}\|\bar{v}\|_{L^{2}}^{2}+\left\|v_{L} \otimes v_{L}\right\|_{\dot{B}_{2, \frac{p}{2}}^{1} \frac{p}{2}}^{2-}\right) .
\end{aligned}
$$

Substituting the above estimates into (4.9) gives rise to

$$
\frac{d}{d t}\|\sqrt{\rho} \bar{v}(t)\|_{L^{2}}^{2}+\|\nabla \bar{v}\|_{L^{2}}^{2} \leq C\left(f_{1}(t)\|\sqrt{\rho} \bar{v}(t)\|_{L^{2}}^{2}+f_{2}(t)\right),
$$


where $f_{1}(t), f_{2}(t)$ are defined by

$$
\begin{aligned}
& f_{1}(t)=\left\|\Delta v_{L}(t)\right\|_{L^{p}}^{r}+\left\|\nabla v_{L}(t)\right\|_{L^{p}}^{\frac{p}{p-1}}+\left\|v_{L} \otimes v_{L}\right\|_{\dot{B}_{2, \frac{p}{2}}^{1}}^{\frac{p}{2}}, \\
& \left.f_{2}(t)=\left\|\Delta v_{L}(t)\right\|_{L^{p}}^{2-\frac{r(p-2)}{p}}+\left\|v_{L} \otimes v_{L}\right\|_{\dot{B}_{2, \frac{p}{2}}^{1}}^{2-\frac{p}{2}} \quad \text { for } r \in\right] 1,2[.
\end{aligned}
$$

By applying Lemma 4.1 and (4.11), we achieve

$$
\begin{aligned}
\int_{0}^{t} f_{1}\left(t^{\prime}\right) d t^{\prime} & \leq C\left(\left\|v_{0}\right\|_{W^{1, p}}^{r}+\left(\int_{0}^{t}\left\|\nabla v_{L}\left(t^{\prime}\right)\right\|_{L^{p}}^{\frac{2 p}{p-1}} d t^{\prime}\right)^{\frac{1}{2}} \sqrt{t}+\left\|v_{0}\right\|_{L^{p}}^{p}\right) \\
& \leq C\left(\left\|v_{0}\right\|_{W^{1, p}}^{r}+\left\|v_{0}\right\|_{W^{1, p}}^{\frac{p}{p-1}} \sqrt{t}+\left\|v_{0}\right\|_{L^{p}}^{p}\right),
\end{aligned}
$$

and since $p \in] 2,4[, r \in] 1,2\left[\right.$, we have $\left.2-\frac{r(p-2)}{p} \in\right] 1,2[$ and

$$
\begin{aligned}
\int_{0}^{t} f_{2}\left(t^{\prime}\right) d t^{\prime} & \leq C\left\|v_{0}\right\|_{W^{1, p}}^{2-\frac{r(p-2)}{p}}+C\left(\int_{0}^{t}\left\|v_{L} \otimes v_{L}\left(t^{\prime}\right)\right\|_{\dot{B}_{2, \frac{p}{2}}^{1}}^{\frac{p}{2}} d t^{\prime}\right)^{\frac{4-p}{p}} t^{2\left(1-\frac{2}{p}\right)} \\
& \leq C\left(\left\|v_{0}\right\|_{W^{1, p}}^{2-\frac{r(p-2)}{p}}+\left\|v_{0}\right\|_{L^{p}}^{8-2 p} t^{2\left(1-\frac{2}{p}\right)}\right) .
\end{aligned}
$$

Therefore by applying Gronwall's inequality to (4.12), we obtain (4.6).

Let us now turn to the proof of (4.11).

Proof of (4.11). We first get, by applying Bony's decomposition (2.4) in the homogeneous context to $v_{L} \otimes v_{L}$, that

$$
v_{L} \otimes v_{L}=2 \dot{T}_{v_{L}} v_{L}+\dot{R}\left(v_{L}, v_{L}\right) .
$$

Due to $v_{0} \in \dot{B}_{p, p}^{0}$ and $\left.p \in\right] 2,4[$, we deduce from Lemma 2.1 that

$$
\begin{aligned}
\left\|\dot{S}_{j^{\prime}-1} v_{L}\right\|_{L^{\frac{2 p}{p-2}}} \lesssim \sum_{\ell \leq j^{\prime}-2} 2^{\ell\left(\frac{4}{p}-1\right)} c_{\ell, p}\left\|v_{0}\right\|_{\dot{B}_{p, p}^{0}} \lesssim c_{j^{\prime}, p} 2^{j^{\prime}\left(\frac{4}{p}-1\right)}\left\|v_{0}\right\|_{L^{p}} \quad \text { and } \\
\left\|\dot{\Delta}_{j^{\prime}} v_{L}\right\|_{L_{t}^{\frac{p}{2}}\left(L^{p}\right)} \lesssim\left\|e^{-c 2^{2 j^{\prime}} t} \dot{\Delta}_{j^{\prime}} v_{0}\right\|_{L_{t}^{\frac{p}{2}}\left(L^{p}\right)} \lesssim c_{j^{\prime}, p} 2^{-\frac{4 j^{\prime}}{p}}\left\|v_{0}\right\|_{L^{p}},
\end{aligned}
$$

from which, we infer

$$
\begin{aligned}
\left\|\dot{\Delta}_{j}\left(\dot{T}_{v_{L}} v_{L}\right)\right\|_{L_{t}^{\frac{p}{2}\left(L^{2}\right)}} & \lesssim \sum_{\left|j^{\prime}-j\right| \leq 4}\left\|\dot{S}_{j^{\prime}-1} v_{L}\right\|_{L_{t}^{\infty}\left(L^{\frac{2 p}{p-2}}\right)}\left\|\dot{\Delta}_{j^{\prime}} v_{L}\right\|_{L_{t}^{\frac{p}{2}}\left(L^{p}\right)} \\
& \lesssim \sum_{\left|j^{\prime}-j\right| \leq 4} c_{j^{\prime}, \frac{p}{2}} 2^{-j^{\prime}}\left\|v_{0}\right\|_{L^{p}}^{2} \lesssim c_{j, \frac{p}{2}} 2^{-j}\left\|v_{0}\right\|_{L^{p}}^{2},
\end{aligned}
$$

where $\left(c_{j, p}\right)_{j \in \mathbb{Z}}$ denotes a unit generic element of $\ell^{p}(\mathbb{Z})$.

Whereas by applying Lemma 2.1 once again, one has

$$
\begin{aligned}
\left\|\dot{\Delta}_{j} \dot{R}\left(v_{L}, v_{L}\right)\right\|_{L_{t}^{\frac{p}{2}}\left(L^{2}\right)} & \lesssim 2^{j^{\prime}\left(\frac{4}{p}-1\right)} \sum_{j^{\prime} \geq j-3}\left\|\dot{\Delta}_{j^{\prime}} v_{L}\right\|_{L_{t}^{\frac{p}{2}}\left(L^{p}\right)}\left\|\widetilde{\dot{\Delta}}_{j^{\prime}} v_{L}\right\|_{L_{t}^{\infty}\left(L^{p}\right)} \\
& \lesssim 2^{j^{\prime}\left(\frac{4}{p}-1\right)} \sum_{j^{\prime} \geq j-3} c_{j^{\prime}, \frac{p}{2}} 2^{-\frac{4 j^{\prime}}{p}}\left\|v_{0}\right\|_{L^{p}}^{2} \\
& \lesssim c_{j, \frac{p}{2}} 2^{-j}\left\|v_{0}\right\|_{L^{p}}^{2} .
\end{aligned}
$$


This proves (4.11).

Lemma 4.2. For any $r \in] 1,2[$ and $p \in] 1, \infty[$, one has

$$
\left\|\int_{0}^{t} \nabla e^{\left(t-t^{\prime}\right) \Delta} f\left(t^{\prime}\right) d t^{\prime}\right\|_{L_{T}^{\frac{2 r}{2-r}\left(L^{p}\right)}}+\left\|\int_{0}^{t} \nabla e^{\left(t-t^{\prime}\right) \Delta} f\left(t^{\prime}\right) d t^{\prime}\right\|_{L_{T}^{q}\left(L^{2 p}\right)} \lesssim\|f\|_{L_{T}^{r}\left(L^{p}\right)},
$$

for $q$ given by $\frac{1}{q}=\frac{1}{r}-\frac{1}{2}\left(1-\frac{1}{p}\right)$.

Proof. As a matter of fact, note that

$$
\begin{aligned}
\nabla e^{\left(t-t^{\prime}\right) \Delta} f\left(t^{\prime}\right) & =\frac{\sqrt{\pi}}{\left(4 \pi\left(t-t^{\prime}\right)\right)^{3 / 2}} \int_{\mathbb{R}^{2}} \frac{x-y}{2\left(t-t^{\prime}\right)^{1 / 2}} \exp \left\{-\frac{|x-y|^{2}}{4\left(t-t^{\prime}\right)}\right\} f\left(t^{\prime}, y\right) d y \\
& \stackrel{\text { def }}{=} \frac{\sqrt{\pi}}{\left(4 \pi\left(t-t^{\prime}\right)\right)^{3 / 2}} K\left(\frac{\cdot}{2\left(t-t^{\prime}\right)^{1 / 2}}\right) * f\left(t^{\prime}, x\right) .
\end{aligned}
$$

Then applying Young's inequality and then Hardy's inequality leads to

$$
\begin{aligned}
\left\|\int_{0}^{t} \nabla e^{\left(t-t^{\prime}\right) \Delta} f\left(t^{\prime}\right) d t^{\prime}\right\|_{L_{T}^{\frac{2 r}{2-r}}\left(L^{p}\right)} & \lesssim\left\|\int_{0}^{t}\left(t-t^{\prime}\right)^{-\frac{1}{2}}\right\| f\left(t^{\prime}\right)\left\|_{L^{p}} d t^{\prime}\right\|_{L_{T}^{2-r}} \\
& \lesssim\|f\|_{L_{T}^{r}\left(L^{p}\right)},
\end{aligned}
$$

and

$$
\begin{aligned}
\left\|\int_{0}^{t} \nabla e^{\left(t-t^{\prime}\right) \Delta} f\left(t^{\prime}\right) d t^{\prime}\right\|_{L_{T}^{q}\left(L^{2 p}\right)} & \lesssim\left\|\int_{0}^{t}\left(t-t^{\prime}\right)^{-\frac{1}{2}\left(1+\frac{1}{p}\right)}\right\| f\left(t^{\prime}\right)\left\|_{L^{p}} d t^{\prime}\right\|_{L_{T}^{q}} \\
& \lesssim\|f\|_{L_{T}^{r}\left(L^{p}\right)}
\end{aligned}
$$

This proves (4.13).

Proposition 4.2. Let $r, \sigma$ be any numbers so that $r \in] 1,2[$ and $\sigma \in] \frac{2 p}{p+2}, p[$. Then under the assumptions of Proposition 4.1, if we assume moreover that $\left\|a_{0}\right\|_{L^{\infty}}$ is sufficiently small, there holds

$$
\begin{aligned}
J_{0} \stackrel{\text { def }}{=} 1+\left\|\left(\partial_{t} v, \Delta v, \nabla \pi\right)\right\|_{L_{t}^{r}\left(L^{p}\right)} & +\|\nabla v\|_{L_{t}^{\sigma}\left(L^{\infty}\right) \cap L_{t}^{\frac{2 r}{2-r}}\left(L^{p}\right)} \\
& +\|v\|_{L_{t}^{\frac{2 r}{2-r}}\left(L^{\infty}\right)}+\|X\|_{L_{t}^{\infty}\left(W^{1, p}\right)} \leq \mathcal{H}_{0}(t) .
\end{aligned}
$$

Proof. We divide the proof of this proposition into the following steps:

- The estimate of $\left\|\left(\partial_{t} v, \Delta v, \nabla \pi\right)\right\|_{L_{t}^{r}\left(L^{p}\right)}$

We first rewrite the momentum equation of (3.1) as

$$
v(t)=e^{t \Delta} v_{0}+\int_{0}^{t} e^{\left(t-t^{\prime}\right) \Delta}(-v \cdot \nabla v+a \Delta v-(1+a) \nabla \pi)\left(t^{\prime}\right) d t^{\prime} .
$$

For any $\lambda>0$, we set

$$
f(t) \stackrel{\text { def }}{=}\|v(t)\|_{L^{2 p}}^{\frac{2 p}{p-1}} \quad \text { and } \quad v_{\lambda}(t) \stackrel{\text { def }}{=} v(t) \exp \left(-\lambda \int_{0}^{t} f\left(t^{\prime}\right) d t^{\prime}\right) .
$$

Then $v_{\lambda}$ solve

$$
v_{\lambda}(t)=e^{t \Delta} v_{0, \lambda}+\int_{0}^{t} e^{-\lambda \int_{t^{\prime}}^{t} f\left(t^{\prime \prime}\right) d t^{\prime \prime}} e^{\left(t-t^{\prime}\right) \Delta}\left(-v \cdot \nabla v_{\lambda}+a \Delta v_{\lambda}-(1+a) \nabla \pi_{\lambda}\right)\left(t^{\prime}\right) d t^{\prime}
$$

Whereas by taking space divergence to the momentum equation of (3.1), one has

$$
\Delta \pi_{\lambda}=-\operatorname{div}\left(v \cdot \nabla v_{\lambda}\right)+\operatorname{div}\left(a\left(\Delta v_{\lambda}-\nabla \pi_{\lambda}\right)\right)
$$


from which and $\left\|a_{0}\right\|_{L^{\infty}}$ is sufficiently small, we infer

$$
\left\|\nabla \pi_{\lambda}(t)\right\|_{L^{p}} \leq C\left(\left\|v \cdot \nabla v_{\lambda}(t)\right\|_{L^{p}}+\left\|a_{0}\right\|_{L^{\infty}}\left\|\Delta v_{\lambda}(t)\right\|_{L^{p}}\right) .
$$

In view of (4.16), we get, by applying maximal regularity estimate to heat semigroup (see Theorem 7.3 of [21] for instance), and the Estimates (4.1), (4.13) and (4.18), that

$$
\begin{gathered}
\left\|\Delta v_{\lambda}\right\|_{L_{t}^{r}\left(L^{p}\right)}+\left\|\left(\partial_{t} v\right)_{\lambda}\right\|_{L_{t}^{r}\left(L^{p}\right)}+\left\|\nabla v_{\lambda}\right\|_{L_{t}^{2-r}\left(L^{p}\right)}+\left\|\nabla v_{\lambda}\right\|_{L_{t}^{q}\left(L^{2 p}\right)} \\
\leq\left\|\Delta v_{L}\right\|_{L_{t}^{r}\left(L^{p}\right)}+\left\|\partial_{t} v_{L}\right\|_{L_{t}^{r}\left(L^{p}\right)}+\left\|\nabla v_{L}\right\|_{L_{t}^{2 r}\left(L^{p}\right)}^{2-r}+\left\|\nabla v_{L}\right\|_{L_{t}^{q}\left(L^{2 p}\right)} \\
+C\left(\int _ { 0 } ^ { t } e ^ { - \lambda r \int _ { t ^ { \prime } } ^ { t } f ( t ^ { \prime \prime } ) d t ^ { \prime \prime } } \left(\left\|v \cdot \nabla v_{\lambda}\left(t^{\prime}\right)\right\|_{L^{p}}^{r}+\left\|a\left(t^{\prime}\right)\right\|_{L^{\infty}}^{r}\left\|\Delta v_{\lambda}\left(t^{\prime}\right)\right\|_{L^{p}}^{r}\right.\right. \\
\left.\left.+\left(1+\left\|a\left(t^{\prime}\right)\right\|_{L^{\infty}}^{r}\right)\left\|\nabla \pi\left(t^{\prime}\right)\right\|_{L^{p}}^{r}\right) d t^{\prime}\right)^{\frac{1}{r}} \\
\leq C\left(\left\|v_{0}\right\|_{W^{1, p}}+\left\|a_{0}\right\|_{L^{\infty}}\left\|\Delta v_{\lambda}\right\|_{L_{t}^{r}\left(L^{p}\right)}\right. \\
\left.+\left(\int_{0}^{t} e^{-\lambda r \int_{t^{\prime}}^{t} f\left(t^{\prime \prime}\right) d t^{\prime \prime}}\left\|v \cdot \nabla v_{\lambda}\left(t^{\prime}\right)\right\|_{L^{p}}^{r} d t^{\prime}\right)^{\frac{1}{r}}\right)
\end{gathered}
$$

where $\frac{1}{q}=\frac{1}{r}-\frac{1}{2}\left(1-\frac{1}{p}\right)$ and thus $\left.q \in\right] \frac{2 p}{p+1}, 2 p\left[\right.$. Hence whenever $\left\|a_{0}\right\|_{L^{\infty}}$ is sufficiently small, we obtain

$$
\begin{aligned}
\left\|\Delta v_{\lambda}\right\|_{L_{t}^{r}\left(L^{p}\right)} & +\left\|\left(\partial_{t} v\right)_{\lambda}\right\|_{L_{t}^{r}\left(L^{p}\right)}+\left\|\nabla v_{\lambda}\right\|_{L_{t}^{\frac{2 r}{2-r}}\left(L^{p}\right)}+\left\|\nabla v_{\lambda}\right\|_{L_{t}^{q}\left(L^{2 p}\right)} \\
& \leq C\left(\left\|v_{0}\right\|_{W^{1, p}}+\left(\int_{0}^{t} e^{-\lambda r \int_{t^{\prime}}^{t} f\left(t^{\prime \prime}\right) d t^{\prime \prime}}\left\|v \cdot \nabla v_{\lambda}\left(t^{\prime}\right)\right\|_{L^{p}}^{r} d t^{\prime}\right)^{\frac{1}{r}}\right) .
\end{aligned}
$$

Whereas due to $\frac{1}{r}=\frac{1}{q}+\frac{1}{2}\left(1-\frac{1}{p}\right)$, it is easy to observe that

$$
\begin{aligned}
& \left(\int_{0}^{t} e^{-\lambda r \int_{t^{\prime}}^{t} f\left(t^{\prime \prime}\right) d t^{\prime \prime}}\left\|v \cdot \nabla v_{\lambda}\left(t^{\prime}\right)\right\|_{L^{p}}^{r} d t^{\prime}\right)^{\frac{1}{r}} \\
& \quad \leq\left(\int_{0}^{t} e^{-\frac{2 p \lambda}{p-1} \int_{t^{\prime}}^{t} f\left(t^{\prime \prime}\right) d t^{\prime \prime}}\left\|v\left(t^{\prime}\right)\right\|_{L^{2 p}}^{\frac{2 p}{p-1}} d t^{\prime}\right)^{\frac{p-1}{2 p}}\left\|\nabla v_{\lambda}\right\|_{L_{t}^{q}\left(L^{2 p}\right)} \\
& \quad \leq \frac{C}{\lambda^{\frac{p-1}{2 p}}}\left\|\nabla v_{\lambda}\right\|_{L_{t}^{q}\left(L^{2 p}\right)} .
\end{aligned}
$$

As a consequence, we obtain

$$
\begin{aligned}
\left\|\Delta v_{\lambda}\right\|_{L_{t}^{r}\left(L^{p}\right)}+\left\|\left(\partial_{t} v\right)_{\lambda}\right\|_{L_{t}^{r}\left(L^{p}\right)}+\left\|\nabla v_{\lambda}\right\|_{L_{t}^{\frac{2 r}{2-r}}\left(L^{p}\right)} & +\left\|\nabla v_{\lambda}\right\|_{L_{t}^{q}\left(L^{2 p}\right)} \\
& \leq C\left(\left\|v_{0}\right\|_{W^{1, p}}+\frac{1}{\lambda^{\frac{p-1}{2 p}}}\left\|\nabla v_{\lambda}\right\|_{L_{t}^{q}\left(L^{2 p}\right)}\right) .
\end{aligned}
$$

Then in view of (4.15), by taking $\lambda$ to be sufficiently large in (4.19), we achieve

$$
\begin{aligned}
& e^{-\lambda \int_{0}^{t} f\left(t^{\prime}\right) d t^{\prime}}\left(\|\Delta v\|_{L_{t}^{r}\left(L^{p}\right)}+\left\|\partial_{t} v\right\|_{L_{t}^{r}\left(L^{p}\right)}+\|\nabla v\|_{L_{t}^{\frac{2 r}{2-r}}\left(L^{p}\right)}+\|\nabla v\|_{L_{t}^{q}\left(L^{2 p}\right)}\right) \\
& \leq\left\|\Delta v_{\lambda}\right\|_{L_{t}^{r}\left(L^{p}\right)}+\left\|\left(\partial_{t} v\right)_{\lambda}\right\|_{L_{t}^{r}\left(L^{p}\right)}+\left\|\nabla v_{\lambda}\right\|_{L_{t}^{2-r}\left(L^{p}\right)}+\left\|\nabla v_{\lambda}\right\|_{L_{t}^{q}\left(L^{2 p}\right)} \leq C\left\|v_{0}\right\|_{W^{1, p}} .
\end{aligned}
$$


While it follows from (4.6) and $v=\bar{v}+v_{L}$ that

$$
\begin{aligned}
\|v\|_{L_{t}^{\frac{2 p}{p-1}\left(L^{2 p}\right)}}^{\frac{2 p}{p-1}} & \leq \int_{0}^{t}\left(\left\|\bar{v}\left(t^{\prime}\right)\right\|_{L^{2 p}}+\left\|v_{L}\left(t^{\prime}\right)\right\|_{L^{2 p}}\right)^{\frac{2 p}{p-1}} d t^{\prime} \\
& \leq C\left(\|\bar{v}\|_{L_{t}^{\infty}\left(L^{2}\right)}^{\frac{2}{p-1}}\|\nabla \bar{v}\|_{L_{t}^{2}\left(L^{2}\right)}^{2}+t\left\|v_{L}\right\|_{L_{t}^{\infty}\left(L^{p}\right)}^{2}\left\|\nabla v_{L}\right\|_{L_{t}^{\infty}\left(L^{p}\right)}^{\frac{2}{p-1}}\right) \\
& \leq C\left(\mathrm{~h}^{\frac{2 p}{p-1}}(t)+t\left\|v_{0}\right\|_{L^{p}}^{2}\left\|\nabla v_{0}\right\|_{L^{p}}^{\frac{2}{p-1}}\right) \leq \mathcal{H}_{0}(t),
\end{aligned}
$$

so that we obtain

$$
\begin{aligned}
\|\Delta v\|_{L_{t}^{r}\left(L^{p}\right)}+\left\|\partial_{t} v\right\|_{L_{t}^{r}\left(L^{p}\right)} & +\|\nabla v\|_{L_{t}^{\frac{2 r}{2-r}}\left(L^{p}\right)}+\|\nabla v\|_{L_{t}^{q}\left(L^{2 p}\right)} \\
& \leq C e^{\lambda \int_{0}^{t} f\left(t^{\prime}\right) d t^{\prime}}\left\|v_{0}\right\|_{W^{1, p}} \leq C e^{\lambda \mathcal{H}_{0}(t)}\left\|v_{0}\right\|_{W^{1, p}} \leq \mathcal{H}_{0}(t) .
\end{aligned}
$$

This together with (4.18) ensures that

$$
\|\nabla \pi\|_{L_{t}^{r}\left(L^{p}\right)} \leq C\left(\|v\|_{L_{t}^{\frac{2 p}{p-1}\left(L^{2 p}\right)}}\|\nabla v\|_{L_{t}^{q}\left(L^{2 p}\right)}+\|\Delta v\|_{L_{t}^{r}\left(L^{p}\right)}\right) \leq \mathcal{H}_{0}(t) .
$$

Moreover, there holds

$$
\left.\|\nabla v\|_{L_{t}^{\frac{2 p r}{2(p+r)-p r}}\left(L^{\infty}\right)} \leq C\|\nabla v\|_{L_{t}^{\frac{2 r}{2-r}}\left(L^{p}\right)}^{1-\frac{2}{p}}\|\Delta v\|_{L_{t}^{r}\left(L^{p}\right)}^{\frac{2}{p}} \leq \mathcal{H}_{0}(t) \quad \text { for } \forall r \in\right] 1,2[.
$$

It is easy to observe that when $r$ varies from 1 to 2 , we have $\left.\sigma=\frac{2 p r}{2(p+r)-p r} \in\right] \frac{2 p}{2+p}, p[$.

- The estimate of $\|v\| \frac{2 r}{L_{t}^{2-r}}\left(L^{\infty}\right)$

In view of (4.8), we get by a similar derivation of (4.16) that

$\bar{v}_{\lambda}(t)=-\int_{0}^{t} e^{-\lambda \int_{t^{\prime}}^{t} f\left(t^{\prime \prime}\right) d t^{\prime \prime}} e^{\left(t-t^{\prime}\right) \Delta} \mathbb{P}\left((\rho-1)\left(\partial_{t} \bar{v}+\partial_{t} v_{L}\right)_{\lambda}+\rho v \cdot \nabla \bar{v}_{\lambda}+\rho v \cdot \nabla v_{L, \lambda}\right)\left(t^{\prime}\right) d t^{\prime}$

where $\mathbb{P} \stackrel{\text { def }}{=} I d-\nabla \Delta^{-1}$ div denotes the Leray projection operator to the divergence free vector space. Then applying the maximal regularity estimate to heat semi-group and Lemma 4.2 yields

$$
\begin{aligned}
& \left\|\Delta \bar{v}_{\lambda}\right\|_{L_{t}^{r}\left(L^{p}\right)}+\left\|\left(\partial_{t} \bar{v}\right)_{\lambda}\right\|_{L_{t}^{r}\left(L^{p}\right)}+\left\|\nabla \bar{v}_{\lambda}\right\|_{L_{t}^{\frac{2 r}{2-r}}\left(L^{p}\right)} \\
& \leq C\left(\left\|(\rho-1)\left(\partial_{t} \bar{v}\right)_{\lambda}\right\|_{L_{t}^{r}\left(L^{p}\right)}+\left\|(\rho-1) \partial_{t} v_{L}\right\|_{L_{t}^{r}\left(L^{p}\right)}\right) \\
& \quad+C\left(\int_{0}^{t} e^{-\lambda r \int_{t^{\prime}}^{t} f\left(t^{\prime \prime}\right) d t^{\prime \prime}}\left(\left\|v \cdot \nabla \bar{v}_{\lambda}\left(t^{\prime}\right)\right\|_{L^{p}}^{r}+\left\|v \cdot \nabla v_{L, \lambda}\left(t^{\prime}\right)\right\|_{L^{p}}^{r}\right) d t^{\prime}\right)^{\frac{1}{r}} .
\end{aligned}
$$

By using Sobolev embedding Theorem, one has

$$
\left\|v \cdot \nabla \bar{v}_{\lambda}\left(t^{\prime}\right)\right\|_{L^{p}} \leq C\left\|v\left(t^{\prime}\right)\right\|_{L^{2 p}}\left\|\nabla \bar{v}_{\lambda}\left(t^{\prime}\right)\right\|_{L^{p}}^{1-\frac{1}{p}}\left\|\Delta \bar{v}_{\lambda}\left(t^{\prime}\right)\right\|_{L^{p}}^{\frac{1}{p}}
$$


so that thanks to (4.15), we get, by applying Hölder's inequality, that

$$
\begin{aligned}
& \left(\int_{0}^{t} e^{-\lambda r \int_{t^{\prime}}^{t} f\left(t^{\prime \prime}\right) d t^{\prime \prime}}\left\|v \cdot \nabla \bar{v}_{\lambda}\left(t^{\prime}\right)\right\|_{L^{p}}^{r} d t^{\prime}\right)^{\frac{1}{r}} \\
& \quad \leq C\left(\int_{0}^{t} e^{-\frac{\lambda p r}{p-1} \int_{t^{\prime}}^{t} f\left(t^{\prime \prime}\right) d t^{\prime \prime}}\left\|v\left(t^{\prime}\right)\right\|_{L^{2 p}}^{\frac{p r}{p-1}}\left\|\nabla \bar{v}_{\lambda}\left(t^{\prime}\right)\right\|_{L^{p}}^{r} d t^{\prime}\right)^{\frac{1}{r}}+\frac{1}{4}\left\|\Delta \bar{v}_{\lambda}\left(t^{\prime}\right)\right\|_{L_{t}^{r}\left(L^{p}\right)} \\
& \quad \leq C\left(\int_{0}^{t} e^{-\frac{2 p \lambda}{p-1} \int_{t^{\prime}}^{t} f\left(t^{\prime \prime}\right) d t^{\prime \prime}}\left\|v\left(t^{\prime}\right)\right\|_{L^{2 p}}^{\frac{2 p}{p-1}} d t^{\prime}\right)^{\frac{1}{2}}\left\|\nabla \bar{v}_{\lambda}\left(t^{\prime}\right)\right\|_{L_{t}^{\frac{2 r}{2-r}}\left(L^{p}\right)}+\frac{1}{4}\left\|\Delta \bar{v}_{\lambda}\left(t^{\prime}\right)\right\|_{L_{t}^{r}\left(L^{p}\right)} \\
& \quad \leq \frac{C}{\lambda^{\frac{1}{2}}}\left\|\nabla \bar{v}_{\lambda}\right\|_{L_{t}^{\frac{2 r}{2-r}}\left(L^{p}\right)}+\frac{1}{4}\left\|\Delta \bar{v}_{\lambda}\right\|_{L_{t}^{r}\left(L^{p}\right) .}
\end{aligned}
$$

Along the same line, we have

$$
\left(\int_{0}^{t} e^{-\lambda r \int_{t^{\prime}}^{t} f\left(t^{\prime \prime}\right) d t^{\prime \prime}}\left\|v \cdot \nabla v_{L, \lambda}\left(t^{\prime}\right)\right\|_{L^{p}}^{r} d t^{\prime}\right)^{\frac{1}{r}} \leq \frac{C}{\lambda^{\frac{1}{2}}}\left\|\nabla v_{L}\right\|_{L_{t}^{\frac{2 r}{2-r}}\left(L^{p}\right)}+\frac{1}{4}\left\|\Delta v_{L}\right\|_{L_{t}^{r}\left(L^{p}\right)} .
$$

As a consequence, we obtain

$$
\begin{aligned}
& \left\|\Delta \bar{v}_{\lambda}\right\|_{L_{t}^{r}\left(L^{p}\right)}+\left\|\left(\partial_{t} \bar{v}\right)_{\lambda}\right\|_{L_{t}^{r}\left(L^{p}\right)}+\left\|\nabla \bar{v}_{\lambda}\right\|_{L_{t}^{\frac{2 r}{2-r}}}\left(L^{p}\right) \\
& \leq C\left(\left\|\rho_{0}-1\right\|_{L^{\infty}}\left\|\left(\partial_{t} \bar{v}\right)_{\lambda}\right\|_{L_{t}^{r}\left(L^{p}\right)}+\frac{1}{\lambda^{\frac{1}{2}}}\left\|\nabla v_{L}\right\|_{L_{t}^{\frac{2 r}{2-r}}\left(L^{p}\right)}+\left\|\Delta v_{L}\right\|_{L_{t}^{r}\left(L^{p}\right)}+\frac{1}{\lambda^{\frac{1}{2}}}\left\|\nabla \bar{v}_{\lambda}\right\|_{L_{t}^{\frac{2 r}{2-r}}\left(L^{p}\right)}\right) .
\end{aligned}
$$

Therefore, by virtue of (4.1), by taking $\lambda$ large enough and $\left\|\rho_{0}-1\right\|_{L^{\infty}}$ to be sufficiently small, we achieve

$$
\left\|\Delta \bar{v}_{\lambda}\right\|_{L_{t}^{r}\left(L^{p}\right)}+\left\|\left(\partial_{t} \bar{v}\right)_{\lambda}\right\|_{L_{t}^{r}\left(L^{p}\right)}+\left\|\nabla \bar{v}_{\lambda}\right\|_{L_{t}^{2-r}\left(L^{p}\right)} \leq C\left\|v_{0}\right\|_{W^{1, p}} .
$$

This in particular implies

$$
\|\nabla \bar{v}\|_{L_{t}^{\frac{2 r}{2-r}}\left(L^{p}\right)} \leq C e^{\lambda \int_{0}^{t} f\left(t^{\prime}\right) d t^{\prime}}\left\|v_{0}\right\|_{W^{1, p}} \leq C \mathcal{H}_{0}(t),
$$

from which, (4.6) and the interpolation inequality that

$$
\|f\|_{L^{\infty}} \leq C\|f\|_{L^{2}}^{\frac{p-2}{2(p-1)}}\|\nabla f\|_{L^{p}}^{\frac{p}{2(p-1)}}
$$

we infer

$$
\|\bar{v}\|_{L_{t}^{\frac{2 r}{2-r}}\left(L^{\infty}\right)} \leq C\|\bar{v}\|_{L_{t}^{\frac{2 r}{2-r}}\left(L^{2}\right)}^{\frac{p-2}{2(p-1)}}\|\nabla \bar{v}\|_{L_{t}^{\frac{2 r}{2-r}}\left(L^{p}\right)}^{\frac{p}{2(p-1)}} \leq C \mathcal{H}_{0}(t) .
$$

While it is easy to notice that

$$
\left\|v_{L}\right\|_{L_{t}^{\infty}\left(L^{\infty}\right)} \leq C\left\|v_{L}\right\|_{L_{t}^{\infty}\left(L^{p}\right)}^{1-\frac{2}{p}}\left\|\nabla v_{L}\right\|_{L_{t}^{\infty}\left(L^{p}\right)}^{\frac{2}{p}} \leq C\left\|v_{0}\right\|_{W^{1, p}},
$$

this together with (4.23) ensures that

$$
\|v\|_{L_{t}^{\frac{2 r}{2-r}}\left(L^{\infty}\right)} \leq C \mathcal{H}_{0}(t)
$$

- The estimate of $\|X\|_{L_{t}^{\infty}\left(W^{1, p}\right)}$

$\overline{\text { Finally, as } X \text { satisfies (3.6), }}$ it follows from standard theory on transport equation and (4.22) that

$$
\|X(t)\|_{L^{p} \cap L^{\infty}} \leq\left\|X_{0}\right\|_{L^{p} \cap L^{\infty}} \exp \left(\int_{0}^{t}\left\|\nabla v\left(t^{\prime}\right)\right\|_{L^{\infty}} d t^{\prime}\right) \leq \mathcal{H}_{0}(t) .
$$


By taking $\partial_{i}, i=1,2$, to $(3.6)$, we get

$$
\partial_{t} \partial_{i} X+v \cdot \nabla \partial_{i} X+\partial_{i} v \cdot \nabla X=\partial_{i} X \cdot \nabla v+X \cdot \nabla \partial_{i} v,
$$

from which, we get, by applying standard $L^{p}$ type energy estimate, that

$$
\frac{d}{d t}\|\nabla X(t)\|_{L^{p}} \leq 2\|\nabla v(t)\|_{L^{\infty}}\|\nabla X(t)\|_{L^{p}}+\|X(t)\|_{L^{\infty}}\left\|\nabla^{2} v\right\|_{L^{p}} .
$$

Applying Gronwall's inequality and using (4.22), (4.25) yields

$$
\begin{aligned}
\|\nabla X(t)\|_{L^{p}} & \leq\left(\left\|\nabla X_{0}\right\|_{L^{p}}+\|X\|_{L_{t}^{\infty}\left(L^{\infty}\right)}\left\|\nabla^{2} v\right\|_{L_{t}^{1}\left(L^{p}\right)}\right) \exp \left(2 \int_{0}^{t}\left\|\nabla v\left(t^{\prime}\right)\right\|_{L^{\infty}} d t^{\prime}\right) \\
& \leq\left(\left\|\nabla X_{0}\right\|_{L^{p}}+\left\|X_{0}\right\|_{L^{\infty}}\left\|\nabla^{2} v\right\|_{L_{t}^{1}\left(L^{p}\right)}\right) \exp \left(3 \int_{0}^{t}\left\|\nabla v\left(t^{\prime}\right)\right\|_{L^{\infty}} d t^{\prime}\right) \leq \mathcal{H}_{0}(t) .
\end{aligned}
$$

This completes the proof of (4.14) and hence the proposition.

\section{The estimates of $\Delta \partial_{X} v, \partial_{t} \partial_{X} v, \nabla \partial_{X} v$}

In this section, we investigate the estimates of $\Delta \partial_{X} v, \partial_{t} \partial_{X} v, \nabla \partial_{X} v$ under the striated regularity assumption of the initial data:

$$
\left.\partial_{X_{0}}^{\ell} a_{0} \in L^{\infty}, \partial_{X_{0}}^{\ell} v_{0} \in W^{1-\frac{\ell}{k} \varepsilon, p}, \partial_{X_{0}}^{\ell-1} X_{0} \in W^{2, p}, \quad \varepsilon \in\right] 0,1[, \forall \ell=1, \cdots, k .
$$

Lemma 5.1. Let $\left.r_{\ell} \in\right] 1, \frac{2 k}{k+\ell \varepsilon}\left[\right.$ and $\left.s_{\ell} \in\right] 2, \frac{2 k}{\ell \varepsilon}[$. Then under the assumption of (5.1), one has

$$
\left\|\Delta e^{t \Delta} \partial_{X_{0}}^{\ell} v_{0}\right\|_{L_{t}^{r \ell}\left(L^{p}\right)}+\left\|\nabla e^{t \Delta} \partial_{X_{0}}^{\ell} v_{0}\right\|_{L_{t}^{s} \ell\left(L^{p}\right)} \leq C\left\|\partial_{X_{0}}^{\ell} v_{0}\right\|_{W^{1-\frac{\ell}{k} \varepsilon, p}}, \quad \forall \ell=1, \cdots, k .
$$

Proof. Since $\partial_{X_{0}}^{\ell} v_{0} \in W^{1-\frac{\ell}{k} \varepsilon, p} \hookrightarrow \dot{B}_{p, p}^{0} \cap \dot{B}_{p, p}^{1-\frac{\ell}{k} \varepsilon}$, so that $\nabla \partial_{X_{0}}^{\ell} v_{0} \in \dot{B}_{p, p}^{-1} \cap \dot{B}_{p, p}^{-\frac{\ell}{k} \varepsilon}$ and $\Delta \partial_{X_{0}} v_{0} \in$ $\dot{B}_{p, p}^{-2} \cap \dot{B}_{p, p}^{-1-\frac{\ell}{k} \varepsilon}$. And therefore, according to Theorem 2.34 of [5], we infer

$$
\begin{aligned}
& \left\|\Delta e^{t \Delta} \partial_{X_{0}}^{\ell} v_{0}\right\|_{L_{t}^{r \ell}\left(L^{p}\right)} \\
& \leq\left\|t^{\frac{1}{2}\left(1+\frac{\ell}{k} \varepsilon\right)-\frac{1}{p}} \Delta e^{t \Delta} \partial_{X_{0}}^{\ell} v_{0}\right\|_{L^{p}(] 0, t \wedge 1\left[\times \mathbb{R}^{2}\right)}\left\|t^{-\left(\frac{1}{2}\left(1+\frac{\ell}{k} \varepsilon\right)-\frac{1}{p}\right)}\right\|_{L^{\bar{r}} \ell(] 0, t \wedge 1[)} \\
& +\left\|t^{1-\frac{1}{p}} \Delta e^{t \Delta} \partial_{X_{0}}^{\ell} v_{0}\right\|_{L^{p}(] t \wedge 1, t\left[\times \mathbb{R}^{2}\right)}\left\|t^{-\left(1-\frac{1}{p}\right)}\right\|_{L^{\bar{r}_{\ell}(] t \wedge 1, t[)}} \\
& \leq C\left(\left\|\Delta \partial_{X_{0}}^{\ell} v_{0}\right\|_{\dot{B}_{p, p}^{-1-\frac{\ell}{k} \varepsilon}}+\left\|\Delta \partial_{X_{0}}^{\ell} v_{0}\right\|_{\dot{B}_{p, p}^{-2}}\right) \leq\left\|\partial_{X_{0}}^{\ell} v_{0}\right\|_{W^{1-\frac{\ell}{k} \varepsilon, p}},
\end{aligned}
$$

where $\bar{r}_{\ell}=\frac{r_{\ell} p}{p-r_{\ell}}$, and we used the fact that $\bar{r}_{\ell}\left(\frac{1}{2}\left(1+\frac{\ell}{k} \varepsilon\right)-\frac{1}{p}\right)<1, \bar{r}_{\ell}\left(1-\frac{1}{p}\right)>1$ for any $\left.r_{\ell} \in\right] 1, \frac{2 k}{k+\ell \varepsilon}[$.

Similarly, one has

$$
\begin{aligned}
\left\|\nabla e^{t \Delta} \partial_{X_{0}}^{\ell} v_{0}\right\|_{L_{t}^{s_{\ell}\left(L^{p}\right)} \leq} \leq & \left\|t^{\frac{\ell}{2 k} \varepsilon-\frac{1}{p}} \nabla e^{t \Delta} \partial_{X_{0}}^{\ell} v_{0}\right\|_{L^{p}(] 0, t \wedge 1\left[\times \mathbb{R}^{2}\right)}\left\|t^{-\left(\frac{\ell}{2 k} \varepsilon-\frac{1}{p}\right)}\right\|_{\left.L^{\bar{s} \ell} \ell\right] 0, t \wedge 1[)} \\
& +\left\|t^{\frac{1}{2}-\frac{1}{p}} \nabla e^{t \Delta} \partial_{X_{0}}^{\ell} v_{0}\right\|_{L^{p}(] t \wedge 1, t\left[\times \mathbb{R}^{2}\right)}\left\|t^{-\left(\frac{1}{2}-\frac{1}{p}\right)}\right\|_{L^{\bar{s}_{\ell}(] t \wedge 1, t[)}} \\
\leq & C\left(\left\|\nabla \partial_{X_{0}}^{\ell} v_{0}\right\|_{\dot{B}_{p, p}^{-\ell} \frac{\ell}{k}}+\left\|\nabla \partial_{X_{0}}^{\ell} v_{0}\right\|_{\dot{B}_{p, p}^{-1}}\right) \leq\left\|\partial_{X_{0}}^{\ell} v_{0}\right\|_{W^{1-\frac{\ell}{k} \varepsilon, p}},
\end{aligned}
$$

where $\bar{s}_{\ell}=\frac{p s_{\ell}}{p-s_{\ell}}$ and we used the fact that $\bar{s}_{\ell}\left(\frac{1}{2}-\frac{1}{p}\right)>1, \bar{s}_{\ell}\left(\frac{\ell}{2 k} \varepsilon-\frac{1}{p}\right)<1$ for any $\left.s_{\ell} \in\right] 2, \frac{2 k}{\ell \varepsilon}[$.

Combining (5.3) with (5.4), we obtain (5.2). 
Proposition 5.1. Let $r_{1}, s_{1}$ be any numbers with $\left.r_{1} \in\right] 1, \frac{2 k}{k+\varepsilon}\left[, s_{1} \in\right] 2, \frac{2 k}{\varepsilon}[$. Then under the assumptions of Proposition 4.2 and (5.1), one has

$$
\begin{aligned}
J_{1} \stackrel{\text { def }}{=} J_{0}+\left\|\partial_{X} a\right\|_{L_{t}^{\infty}\left(L^{\infty}\right)}+\left\|\left(\partial_{t} \partial_{X} v, \Delta \partial_{X} v, \nabla \partial_{X} \pi\right)\right\|_{L_{t}^{r_{1}}\left(L^{p}\right)} \\
+\left\|\nabla \partial_{X} v\right\|_{L_{t}^{r_{1}}\left(L^{\infty}\right) \cap L_{t}^{s_{1}}\left(L^{p}\right)}+\left\|\partial_{X} v\right\|_{L_{t}^{s_{1}}\left(L^{\infty}\right)} \\
+\left\|\partial_{t} X\right\|_{L_{t}^{s_{1}}\left(W^{1, p}\right)}+\|X\|_{L_{t}^{\infty}\left(W^{2, p}\right)} \leq \mathcal{H}_{1}(t) .
\end{aligned}
$$

Proof. We decompose the proof of this proposition into the following steps:

- The estimate of $\partial_{X} a$ and $\Delta X$

For $\ell=1, \cdots, k$, it follows from the transport equation of $(3.1)$ and (3.6) that $\left[\partial_{X} ; D_{t}\right]=0$ for $D_{t} \stackrel{\text { def }}{=} \partial_{t}+v \cdot \nabla$ and thus

$$
\partial_{t} \partial_{X}^{\ell} a+v \cdot \nabla \partial_{X}^{\ell} a=0
$$

which implies

$$
\left\|\partial_{X}^{\ell} a\right\|_{L_{t}^{\infty}\left(L^{\infty}\right)} \leq\left\|\partial_{X_{0}}^{\ell} a_{0}\right\|_{L^{\infty}}
$$

While we get, by applying the operator $\Delta$ to (3.6), that

$$
\partial_{t} \Delta X+v \cdot \nabla \Delta X+2 \sum_{i=1}^{2} \partial_{i} v \cdot \nabla \partial_{i} X+\Delta v \cdot \nabla X=\Delta \partial_{X} v
$$

By using the standard $L^{p}$ energy estimate to the above equation and interpolation inequality that

$$
\left.\|f\|_{L^{\infty}} \leq\|f\|_{\dot{B}_{p, 1}^{\frac{2}{p}}} \leq C\|f\|_{L^{p}}^{1-\frac{2}{p}}\|\nabla f\|_{L^{p}}^{\frac{2}{p}} \quad \text { for } \forall p \in\right] 2, \infty[
$$

we obtain

$$
\begin{gathered}
\frac{d}{d t}\|\Delta X(t)\|_{L^{p}} \leq 2\|\nabla v(t)\|_{L^{\infty}}\left\|\nabla^{2} X(t)\right\|_{L^{p}}+\|\Delta v(t)\|_{L^{p}}\|\nabla X(t)\|_{L^{\infty}}+\left\|\Delta \partial_{X} v(t)\right\|_{L^{p}} \\
\leq C\left(\|\nabla v(t)\|_{L^{p}}^{1-\frac{2}{p}}\|\Delta v(t)\|_{L^{p}}^{\frac{2}{p}}\|\Delta X(t)\|_{L^{p}}\right. \\
\left.\quad+\|\Delta v(t)\|_{L^{p}}\|\nabla X(t)\|_{L^{p}}^{1-\frac{2}{p}}\|\Delta X(t)\|_{L^{p}}^{\frac{2}{p}}+\left\|\Delta \partial_{X} v(t)\right\|_{L^{p}}\right) \\
\leq C\left(\left(\|\nabla v(t)\|_{L^{p}}+\|\Delta v(t)\|_{L^{p}}\right)\|\Delta X(t)\|_{L^{p}}\right. \\
\left.+\|\Delta v(t)\|_{L^{p}}\|\nabla X(t)\|_{L^{p}}+\left\|\Delta \partial_{X} v(t)\right\|_{L^{p}}\right) .
\end{gathered}
$$

Applying Gronwall's inequality and using (4.14) leads to

$$
\begin{aligned}
&\|\Delta X(t)\|_{L^{p}} \leq C\left(\left\|\Delta X_{0}\right\|_{L^{p}}+\|\Delta v\|_{L_{t}^{1}\left(L^{p}\right)}\|\nabla X\|_{L_{t}^{\infty}\left(L^{p}\right)}+\left\|\Delta \partial_{X} v\right\|_{L_{t}^{1}\left(L^{p}\right)}\right) \\
& \times \exp \left(C\left(\|\nabla v\|_{L_{t}^{1}\left(L^{p}\right)}+\|\Delta v\|_{L_{t}^{1}\left(L^{p}\right)}\right)\right) \\
& \leq C \mathcal{H}_{1}(t)\left(1+\left\|\Delta \partial_{X} v\right\|_{L_{t}^{1}\left(L^{p}\right)}\right)
\end{aligned}
$$

- $\underline{\text { The estimates of } \Delta \partial_{X} v \text { and } \nabla \partial_{X} \pi}$ 
We first get, by applying $\partial_{X}$ to the momentum equation of (1.1), that

$$
\begin{aligned}
& \partial_{t} \partial_{X} v+v \cdot \nabla \partial_{X} v-(1+a)\left(\Delta \partial_{X} v-\nabla \partial_{X} \pi\right) \\
&=\partial_{X} a(\Delta v-\nabla \pi)-(1+a)\left(\left[\Delta ; \partial_{X}\right] v-\sum_{i=1}^{2} \nabla X^{i} \partial_{i} \pi\right) \stackrel{\text { def }}{=} F_{1}(v, \pi) .
\end{aligned}
$$

Taking space divergence to (5.10) gives

$$
\operatorname{div}\left((1+a) \nabla \partial_{X} \pi\right)=\operatorname{div}\left(-\partial_{t}\left(\partial_{X} v\right)-v \cdot \nabla \partial_{X} v+\Delta \partial_{X} v+a \Delta \partial_{X} v+F_{1}\right) .
$$

Due to $\operatorname{div} v=0=\operatorname{div} X$, and

$$
\left[\Delta ; \partial_{X}\right] f=\Delta X \cdot \nabla f+2 \sum_{i=1}^{2} \partial_{i} X \cdot \nabla \partial_{i} f
$$

one has

$$
\begin{aligned}
-\operatorname{div} \partial_{t}\left(\partial_{X} v\right) & =-\operatorname{div}\left(\partial_{t} X \cdot \nabla v+X \cdot \nabla \partial_{t} v\right) \\
& =-\operatorname{div}\left(\partial_{t} X \cdot \nabla v\right)-\operatorname{div}\left(\partial_{t} v \cdot \nabla X\right),
\end{aligned}
$$

and

$$
\begin{aligned}
\operatorname{div} \Delta \partial_{X} v & =\operatorname{div}\left(\left[\Delta ; \partial_{X}\right] v\right)+\operatorname{div}\left(\partial_{X} \Delta v\right) \\
& =\operatorname{div}(\Delta X \cdot \nabla v)+2 \sum_{i=1}^{2} \operatorname{div}\left(\partial_{i} X \cdot \nabla \partial_{i} v\right)+\operatorname{div}(\Delta v \cdot \nabla X)
\end{aligned}
$$

Therefore, one achieves

$$
\begin{aligned}
\operatorname{div}\left((1+a) \nabla \partial_{X} \pi\right)= & -\operatorname{div}\left(\partial_{t} X \cdot \nabla v\right)-\operatorname{div}\left(v \cdot \nabla \partial_{X} v\right)+\operatorname{div}\left(\partial_{X} a(\Delta v-\nabla \pi)\right. \\
& -\left(\partial_{t} v-\Delta v\right) \cdot \nabla X-2 a \nabla X: \nabla^{2} v \\
& +(1+a) \nabla X \cdot \nabla \pi)-\operatorname{div}(a \Delta X \cdot \nabla v)+\operatorname{div}\left(a \Delta \partial_{X} v\right),
\end{aligned}
$$

from which, and $\|a\|_{L^{\infty}}$ is sufficiently small, we infer

$$
\begin{aligned}
\left\|\nabla \partial_{X} \pi(t)\right\|_{L^{p}} \lesssim & \left\|\partial_{t} X \cdot \nabla v(t)\right\|_{L^{p}}+\left\|v \cdot \nabla \partial_{X} v(t)\right\|_{L^{p}} \\
& +\left\|\left(\partial_{t} v, \Delta v, \nabla \pi\right)(t)\right\|_{L^{p}}\left\|\left(\nabla X, \partial_{X} a\right)(t)\right\|_{L^{\infty}} \\
& +\|\nabla v(t)\|_{L^{\infty}}\|\Delta X(t)\|_{L^{p}}+\left\|a_{0}\right\|_{L^{\infty}}\left\|\Delta \partial_{X} v(t)\right\|_{L^{p}}
\end{aligned}
$$

For any $\left.r_{1} \in\right] 1, \frac{2 k}{k+\varepsilon}\left[\right.$, let us take $\left.\left.\left.\sigma_{1}, \sigma_{2} \in\right] \frac{2 p}{p+2}, p\left[, r_{01}, r_{02}, r_{03} \in\right] 1, \infty\right], s_{01}, s_{02} \in\right] 2, \infty[$, and $\left.r_{0} \in\right] 1,2[$ so that

$$
\frac{1}{r_{1}}=\frac{1}{r_{01}}+\frac{1}{\sigma_{1}}+\frac{1}{s_{01}}=\frac{1}{s_{02}}+\frac{1}{r_{03}}+\frac{1}{\sigma_{2}}=\frac{1}{s_{02}}+\frac{1}{r_{02}}+\frac{1}{r_{0}} .
$$

Then by virtue of (4.14) and the equation for $X$ (3.6), we write

$$
\begin{aligned}
& \left\|\partial_{t} X \cdot \nabla v\right\|_{L_{t}^{r_{1}\left(L^{p}\right)}}+\left\|v \cdot \nabla \partial_{X} v\right\|_{L_{t}^{r_{1}\left(L^{p}\right)}} \\
& \quad \lesssim\left\|\partial_{t} X \otimes \nabla v\right\|_{L_{t}^{r_{1}}\left(L^{p}\right)}+\|v \otimes \nabla X \otimes \nabla v\|_{L_{t}^{r_{1}\left(L^{p}\right)}}+\left\|v \otimes X \otimes \nabla^{2} v\right\|_{L_{t}^{r_{1}}\left(L^{p}\right)} \\
& \quad \lesssim\|X\|_{L_{t}^{r_{01}\left(L^{\infty}\right)}\|\nabla v\|_{L_{t}^{\sigma_{1}}\left(L^{\infty}\right)}\|\nabla v\|_{L_{t}^{s_{01}}\left(L^{p}\right)}} \\
& \quad+\|v\|_{L_{t}^{s_{02}\left(L^{\infty}\right)}}\left(\|\nabla X\|_{L_{t}^{r_{03}\left(L^{p}\right)}}\|\nabla v\|_{L_{t}^{\sigma_{2}\left(L^{\infty}\right)}}+\|X\|_{L_{t}^{r_{02}\left(L^{\infty}\right)}}\left\|\nabla^{2} v\right\|_{L_{t}^{r_{0}\left(L^{p}\right)}}\right) \\
& \quad \lesssim \mathcal{H}_{1}(t) .
\end{aligned}
$$


Therefore, taking into account (5.7), we obtain

$$
\begin{aligned}
\left\|\nabla \partial_{X} \pi\right\|_{L_{t}^{r_{1}}\left(L^{p}\right)} \leq & \mathcal{H}_{1}(t)+C\left(\left\|\left(\partial_{t} v, \Delta v, \nabla \pi\right)\right\|_{L_{t}^{r_{1}}\left(L^{p}\right)}\left\|\left(\nabla X, \partial_{X_{0}} a_{0}\right)\right\|_{L_{t}^{\infty}\left(L^{\infty}\right)}\right. \\
& \left.+\left(\int_{0}^{t}\left\|\nabla v\left(t^{\prime}\right)\right\|_{L^{\infty}}^{r_{1}}\left\|\Delta X\left(t^{\prime}\right)\right\|_{L^{p}}^{r_{1}} d t^{\prime}\right)^{\frac{1}{r_{1}}}+\left\|a_{0}\right\|_{L^{\infty}}\left\|\Delta \partial_{X} v\right\|_{L_{t}^{r_{1}}\left(L^{p}\right)}\right) \\
\leq & C\left(\mathcal{H}_{1}(t)\left(1+\|\nabla X\|_{L_{t}^{\infty}\left(L^{\infty}\right)}\right)+\left\|a_{0}\right\|_{L^{\infty}}\left\|\Delta \partial_{X} v\right\|_{L_{t}^{r_{1}}\left(L^{p}\right)}\right. \\
& \left.+\left(\int_{0}^{t}\left\|\nabla v\left(t^{\prime}\right)\right\|_{L^{\infty}}^{r_{1}}\left\|\Delta X\left(t^{\prime}\right)\right\|_{L^{p}}^{r_{1}} d t^{\prime}\right)^{\frac{1}{r_{1}}}\right) .
\end{aligned}
$$

Similarly it follows that

$$
\begin{aligned}
& \left\|F_{1}\right\|_{L_{t}^{r_{1}\left(L^{p}\right)}} \leq\left\|\partial_{X} a\right\|_{L_{t}^{\infty}\left(L^{\infty}\right)}\left(\|\Delta v\|_{L_{t}^{r_{1}\left(L^{p}\right)}}+\|\nabla \pi\|_{L_{t}^{r_{1}\left(L^{p}\right)}}\right) \\
& +\|\nabla X\|_{L_{t}^{\infty}\left(L^{\infty}\right)}\left\|\nabla^{2} v\right\|_{L_{t}^{r_{1}}\left(L^{p}\right)}+\left(\int_{0}^{t}\left\|\nabla v\left(t^{\prime}\right)\right\|_{L^{\infty}}^{r_{1}}\left\|\Delta X\left(t^{\prime}\right)\right\|_{L^{p}}^{r_{1}} d t^{\prime}\right)^{\frac{1}{r_{1}}} \\
& \leq \mathcal{H}_{1}(t)\left(1+\|\nabla X\|_{L_{t}^{\infty}\left(L^{\infty}\right)}\right)+\left(\int_{0}^{t}\left\|\nabla v\left(t^{\prime}\right)\right\|_{L^{\infty}}^{r_{1}}\left\|\Delta X\left(t^{\prime}\right)\right\|_{L^{p}}^{r_{1}} d t^{\prime}\right)^{\frac{1}{r_{1}}} .
\end{aligned}
$$

On the other hand, in view of $(5.10)$, we write

$$
\partial_{X} v(t)=e^{t \Delta} \partial_{X_{0}} v_{0}+\int_{0}^{t} e^{\left(t-t^{\prime}\right) \Delta}\left(-v \cdot \nabla \partial_{X} v+a \Delta \partial_{X} v-(1+a) \nabla \partial_{X} \pi+F_{1}\right)\left(t^{\prime}\right) d t^{\prime}
$$

from which, (5.2) and maximal regularity estimate for heat semi-group, we infer

$$
\begin{aligned}
\left\|\partial_{t} \partial_{X} v\right\|_{L_{t}^{r_{1}}\left(L^{p}\right)}+\left\|\Delta \partial_{X} v\right\|_{L_{t}^{r_{1}}\left(L^{p}\right)} & \\
\leq & \left\|\partial_{X_{0}} v_{0}\right\|_{W^{1-\frac{\varepsilon}{k}, p}}+C\left(\left\|F_{1}\right\|_{L_{t}^{r_{1}}\left(L^{p}\right)}+\left\|v \cdot \nabla \partial_{X} v\right\|_{L_{t}^{r_{1}}\left(L^{p}\right)}\right. \\
& \left.\quad+\|a\|_{L_{t}^{\infty}\left(L^{\infty}\right)}\left\|\Delta \partial_{X} v\right\|_{L_{t}^{r_{1}}\left(L^{p}\right)}+\left(1+\|a\|_{L_{t}^{\infty}\left(L^{\infty}\right)}\right)\left\|\nabla \partial_{X} \pi\right\|_{L_{t}^{r_{1}}\left(L^{p}\right)}\right) .
\end{aligned}
$$

Hence by virtue of (5.12) and (5.13), we get, by taking $\left\|a_{0}\right\|_{L^{\infty}}$ to be sufficiently small, that

$$
\begin{aligned}
\left\|\partial_{t} \partial_{X} v\right\|_{L_{t}^{r_{1}\left(L^{p}\right)}}+\left\|\Delta \partial_{X} v\right\|_{L_{t}^{r_{1}\left(L^{p}\right)}} \leq & \mathcal{H}_{1}(t)\left(1+\|\nabla X\|_{L_{t}^{\infty}\left(L^{\infty}\right)}\right) \\
& +C\left(\int_{0}^{t}\left\|\nabla v\left(t^{\prime}\right)\right\|_{L^{\infty}}^{r_{1}}\left\|\Delta X\left(t^{\prime}\right)\right\|_{L^{p}}^{r_{1}} d t^{\prime}\right)^{\frac{1}{r_{1}}} .
\end{aligned}
$$

Resuming the above estimate into (5.9) and using (5.8) gives rise to

$$
\begin{aligned}
\|\Delta X\|_{L_{t}^{\infty}\left(L^{p}\right) \leq} \leq & \mathcal{H}_{1}(t)\left(1+\|\nabla X\|_{L_{t}^{\infty}\left(L^{p}\right)}^{1-\frac{2}{p}}\|\Delta X\|_{L_{t}^{\infty}\left(L^{p}\right)}^{\frac{2}{p}}\right) \\
& +C t^{1-\frac{1}{r_{1}}}\left(\int_{0}^{t}\left\|\nabla v\left(t^{\prime}\right)\right\|_{L^{\infty}}^{r_{1}}\left\|\Delta X\left(t^{\prime}\right)\right\|_{L^{p}}^{r_{1}} d t^{\prime}\right)^{\frac{1}{r_{1}}} \\
\leq & \mathcal{H}_{1}(t)+\frac{1}{2}\|\Delta X\|_{L_{t}^{\infty}\left(L^{p}\right)}+C t^{1-\frac{1}{r_{1}}}\left(\int_{0}^{t}\left\|\nabla v\left(t^{\prime}\right)\right\|_{L^{\infty}}^{r_{1}}\left\|\Delta X\left(t^{\prime}\right)\right\|_{L^{p}}^{r_{1}} d t^{\prime}\right)^{\frac{1}{r_{1}}},
\end{aligned}
$$

which gives

$$
\|\Delta X\|_{L_{t}^{\infty}\left(L^{p}\right)}^{r_{1}} \leq \mathcal{H}_{1}(t)^{r_{1}}+C t^{r_{1}-1} \int_{0}^{t}\left\|\nabla v\left(t^{\prime}\right)\right\|_{L^{\infty}}^{r_{1}}\left\|\Delta X\left(t^{\prime}\right)\right\|_{L^{p}}^{r_{1}} d t^{\prime} .
$$


Applying Gronwall's inequality and using (4.14) gives

$$
\|\Delta X\|_{L_{t}^{\infty}\left(L^{p}\right)}^{r_{1}} \leq \mathcal{H}(t) \exp \left(C t^{r_{1}-1} \int_{0}^{t}\left\|\nabla v\left(t^{\prime}\right)\right\|_{L^{\infty}}^{r_{1}} d t^{\prime}\right) \leq \mathcal{H}_{1}(t) .
$$

This together with (5.15) and (5.12) ensures that

$$
\left\|\partial_{t} \partial_{X} v\right\|_{L_{t}^{r_{1}\left(L^{p}\right)}}+\left\|\Delta \partial_{X} v\right\|_{L_{t}^{r_{1}}\left(L^{p}\right)}+\left\|\nabla \partial_{X} \pi\right\|_{L_{t}^{r_{1}}\left(L^{p}\right)} \leq \mathcal{H}_{1}(t)
$$

- The estimate of $\nabla \partial_{X} v$ and $\partial_{t} X$

It is easy to observe that for any $\left.s_{1} \in\right] 2, \frac{2 k}{\varepsilon}\left[\right.$, there holds $\left.r_{1}=\frac{2 s_{1}}{2+s_{1}} \in\right] 1, \frac{2 k}{k+\varepsilon}[$. Hence in view of (4.13), (5.2) and (5.14), we get

$$
\begin{aligned}
\left\|\nabla \partial_{X} v\right\|_{L_{t}^{s_{1}}\left(L^{p}\right)} \leq & \left\|\partial_{X_{0}} v_{0}\right\|_{W^{1-\frac{\varepsilon}{k}, p}}+C\left(\left\|F_{1}\right\|_{L_{t}^{r_{1}}\left(L^{p}\right)}+\left\|v \cdot \nabla \partial_{X} v\right\|_{L_{t}^{r_{1}}\left(L^{p}\right)}\right. \\
& \left.+\|a\|_{L_{t}^{\infty}\left(L^{\infty}\right)}\left\|\Delta \partial_{X} v\right\|_{L_{t}^{r_{1}}\left(L^{p}\right)}+\left(1+\|a\|_{L_{t}^{\infty}\left(L^{\infty}\right)}\right)\left\|\nabla \partial_{X} \pi\right\|_{L_{t}^{r_{1}}\left(L^{p}\right)}\right) .
\end{aligned}
$$

which together with (5.17) implies that

$$
\begin{aligned}
& \left\|\nabla \partial_{X} v\right\|_{L_{t}^{s_{1}}\left(L^{p}\right)} \leq \mathcal{H}_{1}(t), \quad \text { and } \\
& \left\|\nabla \partial_{X} v\right\|_{L_{t}^{r_{1}}\left(L^{\infty}\right)} \leq C\left\|\nabla \partial_{X} v\right\|_{L_{t}^{r_{1}}\left(L^{p}\right)}^{1-\frac{2}{p}}\left\|\Delta \partial_{X} v\right\|_{L_{t}^{r_{1}\left(L^{p}\right)}}^{\frac{2}{p}} \leq \mathcal{H}_{1}(t) .
\end{aligned}
$$

Whereas since $\nabla v \in L_{t}^{\frac{2 r}{2-r}}\left(L^{p}\right)$ for all $\left.r \in\right] 1,2\left[\right.$, one has $\partial_{X} v \in L_{t}^{\frac{2 r}{2-r}}\left(L^{p}\right)$ and

$$
\left\|\partial_{X} v\right\|_{L_{t}^{s_{1}}\left(L^{\infty}\right)} \leq C\left\|\partial_{X} v\right\|_{L_{t}^{s_{1}}\left(L^{p}\right)}^{1-\frac{2}{p}}\left\|\nabla \partial_{X} v\right\|_{L_{t}^{s_{1}}\left(L^{p}\right)}^{\frac{2}{p}} \leq \mathcal{H}_{1}(t) .
$$

Moreover, it follows from (3.6) that

$$
\left\|\partial_{t} X\right\|_{L_{t}^{s_{1}}\left(L^{p}\right)} \leq\|v\|_{L_{t}^{s_{1}\left(L^{\infty}\right)}}\|\nabla X\|_{L_{t}^{\infty}\left(L^{p}\right)}+\left\|\partial_{X} v\right\|_{L_{t}^{s_{1}\left(L^{p}\right)}} \leq \mathcal{H}_{1}(t)
$$

and

$$
\begin{aligned}
\left\|\nabla \partial_{t} X\right\|_{L_{t}^{s_{1}}\left(L^{p}\right)} & \lesssim\|\nabla v \otimes \nabla X\|_{L_{t}^{s_{1}}\left(L^{p}\right)}+\left\|v \otimes \nabla^{2} X\right\|_{L_{t}^{s_{1}}\left(L^{p}\right)}+\left\|\nabla \partial_{X} v\right\|_{L_{t}^{s_{1}}\left(L^{p}\right)} \\
& \lesssim\|\nabla v\|_{L_{t}^{s_{1}}\left(L^{p}\right)}\|\nabla X\|_{L_{t}^{\infty}\left(L^{\infty}\right)}+\|v\|_{L_{t}^{s_{1}}\left(L^{\infty}\right)}\left\|\nabla^{2} X\right\|_{L_{t}^{\infty}\left(L^{p}\right)}+\left\|\nabla \partial_{X} v\right\|_{L_{t}^{s_{1}}\left(L^{p}\right)} \\
& \leq \mathcal{H}_{1}(t) .
\end{aligned}
$$

This yields

$$
\left\|\partial_{t} X\right\|_{L_{t}^{s_{1}}\left(W^{1, p}\right)} \leq \mathcal{H}_{1}(t)
$$

This together with (5.16-5.19) leads to (5.5).

\section{The Proof of Theorem 3.1}

Recalling the definitions of $J_{0}, J_{1}$ given by (4.14) and (5.5) respectively, in general for $\ell \geq 2$ and any $\left.r_{\ell} \in\right] 1, \frac{2 k}{k+\ell \varepsilon}\left[, s_{\ell} \in\right] 2, \frac{2 k}{\ell \varepsilon}\left[\right.$, let us define $J_{\ell}(t)$ as follows

$$
\begin{aligned}
& J_{\ell}(t) \stackrel{\text { def }}{=} J_{\ell-1}(t)+\left\|\left(\partial_{t} \partial_{X}^{\ell} v, \Delta \partial_{X}^{\ell} v, \nabla \partial_{X}^{\ell} \pi\right)\right\|_{L_{t}^{r}\left(L^{p}\right)}+\left\|\partial_{X}^{\ell} a\right\|_{L_{t}^{\infty}\left(L^{\infty}\right)} \\
& +\left\|\nabla \partial_{X}^{\ell} v\right\|_{L_{t}^{r \ell}\left(L^{\infty}\right) \cap L_{t}^{s \ell}\left(L^{p}\right)}+\left\|\partial_{X}^{\ell} v\right\|_{L_{t}^{s \ell}\left(L^{\infty}\right)}+\left\|\partial_{t} \partial_{X}^{\ell-1} X\right\|_{L_{t}^{s} \ell\left(W^{1, p}\right)}+\left\|\partial_{X}^{\ell-1} X\right\|_{L_{t}^{\infty}\left(W^{2, p}\right)} .
\end{aligned}
$$

Then Theorem 3.1 essentially follows from the following proposition:

Proposition 6.1. Under the assumptions of Proposition 5.1, one has

$$
J_{\ell}(t) \leq \mathcal{H}_{\ell}(t), \quad \forall \ell=2, \cdots, k .
$$

Its proof will depend on the following three lemmas: 
Lemma 6.1. For any $\left.\ell \in\{1, \cdots, k\}, r_{\ell} \in\right] 1, \frac{2 k}{k+\ell \varepsilon}\left[\right.$, and $\left.s_{\ell} \in\right] 2, \frac{2 k}{\ell \varepsilon}[$, we have

$$
\begin{aligned}
& \left\|\partial_{X}^{i} \nabla \partial_{X}^{\ell-i} X-\nabla \partial_{X}^{\ell} X\right\|_{L_{t}^{\infty}\left(L^{\infty}\right)}+\left\|\partial_{X}^{i} \nabla \partial_{X}^{\ell-i} X-\nabla \partial_{X}^{\ell} X\right\|_{L_{t}^{\infty}\left(W^{1, p}\right)} \\
& +\left\|\partial_{X}^{i} \nabla^{2} \partial_{X}^{\ell-i} X-\nabla^{2} \partial_{X}^{\ell} X\right\|_{L_{t}^{\infty}\left(L^{p}\right)}+\left\|\partial_{X}^{i} \partial_{t} \partial_{X}^{\ell-i} X-\partial_{t} \partial_{X}^{\ell} X\right\|_{L_{t}^{s}\left(W^{1, p}\right)} \lesssim J_{\ell}^{\ell+1} .
\end{aligned}
$$

When $i \neq \ell$, one has

$$
\begin{aligned}
& \left\|\partial_{X}^{i} \nabla \partial_{X}^{\ell-i} v-\nabla \partial_{X}^{\ell} v\right\|_{L_{t}^{r_{\ell-1}}\left(L^{\infty}\right) \cap L_{t}^{s_{\ell-1}}\left(L^{p}\right)}+\left\|\partial_{X}^{i} \nabla^{2} \partial_{X}^{\ell-i} v-\nabla^{2} \partial_{X}^{\ell} v\right\|_{L_{t}^{r_{\ell-1}}\left(L^{p}\right)} \\
& +\left\|\partial_{X}^{i} \nabla \partial_{X}^{\ell-i} \pi-\nabla \partial_{X}^{\ell} \pi\right\|_{L_{t}^{r \ell-1}\left(L^{p}\right)}+\left\|\partial_{X}^{i} \partial_{t} \partial_{X}^{\ell-i} v-\partial_{t} \partial_{X}^{\ell} v\right\|_{L_{t}^{r \ell-1}\left(L^{p}\right)} \lesssim J_{\ell-1}^{\ell+1}
\end{aligned}
$$

and in the case when $i=\ell$, there holds

$$
\begin{aligned}
& \left\|\partial_{X}^{\ell} \nabla v-\nabla \partial_{X}^{\ell} v+\partial_{X}^{\ell-1} \nabla X \cdot \nabla v\right\|_{L_{t}^{r \ell-1}\left(L^{\infty}\right) \cap L_{t}^{s \ell-1}\left(L^{p}\right)} \lesssim J_{\ell-1}^{\ell+1} \\
& \left\|\partial_{X}^{\ell} \Delta v-\Delta \partial_{X}^{\ell} v+\partial_{X}^{\ell-1} \Delta X \cdot \nabla v+2 \partial_{X}^{\ell-1} \nabla X: \nabla^{2} v\right\|_{L_{t}^{r \ell-1}\left(L^{p}\right)} \lesssim J_{\ell-1}^{\ell+1}, \\
& \left\|\partial_{X}^{\ell} \nabla \pi-\nabla \partial_{X}^{\ell} \pi+\partial_{X}^{\ell-1} \nabla X \cdot \nabla \pi\right\|_{L_{t}^{r \ell-1}\left(L^{p}\right)} \lesssim J_{\ell-1}^{\ell+1}, \\
& \left\|\partial_{X}^{\ell} \partial_{t} v-\partial_{t} \partial_{X}^{\ell} v+\partial_{X}^{\ell-1} \partial_{t} X \cdot \nabla v\right\|_{L_{t}^{r \ell-1}\left(L^{p}\right)} \lesssim J_{\ell-1}^{\ell+1} .
\end{aligned}
$$

Remark 6.1. It follows from (6.3), (6.4) and (6.5) that

$$
\begin{aligned}
\left\|\partial_{X}^{i} \nabla \partial_{X}^{\ell-i} X\right\|_{L_{t}^{\infty}\left(L^{\infty}\right)}+\left\|\partial_{X}^{i} \nabla \partial_{X}^{\ell-i} X\right\|_{L_{t}^{\infty}\left(W^{1, p}\right)}, \\
\quad+\left\|\partial_{X}^{i} \nabla^{2} \partial_{X}^{\ell-i} X\right\|_{L_{t}^{\infty}\left(L^{p}\right)}+\left\|\partial_{X}^{i} \partial_{t} \partial_{X}^{\ell-i} X\right\|_{L_{t}^{s \ell+1}\left(W^{1, p}\right)} \lesssim J_{\ell+1}^{\ell+1},
\end{aligned}
$$

and

$$
\begin{aligned}
& \left\|\partial_{X}^{i} \nabla \partial_{X}^{\ell-i} v\right\|_{L_{t}^{r \ell}\left(L^{\infty}\right) \cap L_{t}^{s \ell}\left(L^{p}\right)}+\left\|\partial_{X}^{i} \nabla^{2} \partial_{X}^{\ell-i} v\right\|_{L_{t}^{r_{\ell}}\left(L^{p}\right)} \\
& +\left\|\partial_{X}^{i} \nabla \partial_{X}^{\ell-i} \pi\right\|_{L_{t}^{r \ell}\left(L^{p}\right)}+\left\|\partial_{X}^{i} \partial_{t} \partial_{X}^{\ell-i} v\right\|_{L_{t}^{r \ell}\left(L^{p}\right)} \lesssim J_{\ell}^{\ell+1} .
\end{aligned}
$$

Proof. It is easy to observe that

$$
\begin{aligned}
& \left\|\partial_{X} \nabla X-\nabla \partial_{X} X\right\|_{L_{t}^{\infty}\left(L^{\infty}\right)}+\left\|\partial_{X} \nabla X-\nabla \partial_{X} X\right\|_{L_{t}^{\infty}\left(W^{1, p}\right)} \\
& +\left\|\partial_{X} \nabla^{2} X-\nabla^{2} \partial_{X} X\right\|_{L_{t}^{\infty}\left(L^{p}\right)}+\left\|\partial_{X} \partial_{t} X-\partial_{t} \partial_{X} X\right\|_{L_{t}^{s_{1}}\left(W^{1, p}\right)} \\
& \leq C\|\nabla X\|_{L_{t}^{\infty}\left(W^{1, p}\right)}\left(\|\nabla X\|_{L_{t}^{\infty}\left(W^{1, p}\right)}+\left\|\partial_{t} X\right\|_{L_{t}^{s_{1}}\left(W^{1, p}\right)}\right) \lesssim J_{1}^{2} .
\end{aligned}
$$

This shows that (6.3) holds for $\ell=1$. And (6.4) and (6.5) hold trivially for $\ell=1$. And hence Lemma 6.1 holds for $k=1$.

Let us now assume that (6.3-6.7) hold for $\ell \leq j-1$ for $j \leq k$. We are going to prove that (6.3-6.5) also hold for $\ell=j$, which also implies (6.6) and (6.7) for $\ell=j$. As a matter of fact, it follows from a trivial calculation that for $i \leq j-1$,

$$
\begin{aligned}
\partial_{X}^{i+1} \nabla \partial_{X}^{j-i-1} f & =\nabla \partial_{X}^{j} f+\sum_{m=0}^{i} \partial_{X}^{m}\left[\partial_{X}, \nabla\right] \partial_{X}^{j-m-1} f \\
& =\nabla \partial_{X}^{j} f-\sum_{m=0}^{i} \partial_{X}^{m}\left(\nabla X \cdot \nabla \partial_{X}^{j-m-1} f\right) \\
& =\nabla \partial_{X}^{j} f-\sum_{m=0}^{i} \sum_{n=0}^{m} C_{m}^{n} \partial_{X}^{n} \nabla X \cdot \partial_{X}^{m-n} \nabla \partial_{X}^{j-m-1} f
\end{aligned}
$$


from which and the induction assumptions, we infer

$$
\begin{aligned}
& \left\|\partial_{X}^{i+1} \nabla \partial_{X}^{j-i-1} X-\nabla \partial_{X}^{j} X\right\|_{L_{t}^{\infty}\left(L^{\infty}\right)}+\left\|\partial_{X}^{i+1} \nabla \partial_{X}^{j-i-1} X-\nabla \partial_{X}^{j} X\right\|_{L_{t}^{\infty}\left(W^{1, p}\right)} \\
& \lesssim \sum_{m=0}^{i} \sum_{n=0}^{m}\left\|\partial_{X}^{n} \nabla X\right\|_{L_{t}^{\infty}\left(W^{1, p}\right)}\left\|\partial_{X}^{m-n} \nabla \partial_{X}^{j-m-1} X\right\|_{L_{t}^{\infty}\left(W^{1, p}\right)} \\
& \lesssim \sum_{m=0}^{i} \sum_{n=0}^{m} J_{n+1}^{n+1} J_{j-n}^{j-n} \lesssim J_{j}^{j+1} .
\end{aligned}
$$

By the same manner, it also easy to observe that for $i \leq j-1$,

$$
\begin{aligned}
\partial_{X}^{i+1} \nabla^{2} \partial_{X}^{j-i-1} f & =\nabla^{2} \partial_{X}^{j} f+\sum_{m=0}^{i} \partial_{X}^{m}\left[\partial_{X}, \nabla^{2}\right] \partial_{X}^{j-m-1} f \\
& =\nabla^{2} \partial_{X}^{j} f-\sum_{m=0}^{i} \partial_{X}^{m}\left(\nabla^{2} X \cdot \nabla \partial_{X}^{j-m-1} f+2 \nabla X \cdot \nabla^{2} \partial_{X}^{j-m-1} f\right) \\
& =\nabla^{2} \partial_{X}^{j} f-\sum_{m=0}^{i} \sum_{n=0}^{m} C_{m}^{n}\left(\partial_{X}^{n} \nabla^{2} X \cdot \partial_{X}^{m-n} \nabla \partial_{X}^{j-m-1} f\right. \\
& \left.+2 \partial_{X}^{n}(\nabla X) \cdot \partial_{X}^{m-n} \nabla^{2} \partial_{X}^{j-m-1} f\right)
\end{aligned}
$$

This together with the inductive assumptions ensures that

$$
\begin{aligned}
\| \partial_{X}^{i+1} \nabla^{2} \partial_{X}^{j-i-1} X & -\nabla^{2} \partial_{X}^{j} X \|_{L_{t}^{\infty}\left(L^{p}\right)} \\
\lesssim \sum_{m=0}^{i} \sum_{n=0}^{m}( & \left\|\partial_{X}^{m-n} \nabla \partial_{X}^{j-m-1} X\right\|_{L_{t}^{\infty}\left(L^{\infty}\right)}\left\|\partial_{X}^{n} \nabla^{2} X\right\|_{L_{t}^{\infty}\left(L^{p}\right)} \\
& \left.\quad+\left\|\partial_{X}^{m-n} \nabla^{2} \partial_{X}^{j-m-1} X\right\|_{L_{t}^{\infty}\left(L^{p}\right)}\left\|\partial_{X}^{n} \nabla X\right\|_{L_{t}^{\infty}\left(L^{\infty}\right)}\right) \lesssim J_{j}^{j+1}
\end{aligned}
$$

Finally notice that

$$
\begin{aligned}
\partial_{X}^{i+1} \partial_{t} \partial_{X}^{j-i-1} f & =\partial_{t} \partial_{X}^{j} f+\sum_{m=0}^{i} \partial_{X}^{m}\left[\partial_{X}, \partial_{t}\right] \partial_{X}^{j-m-1} f \\
& =\partial_{t} \partial_{X}^{j} f-\sum_{m=0}^{i} \partial_{X}^{m}\left(\partial_{t} X \cdot \nabla \partial_{X}^{j-m-1} f\right) \\
& =\partial_{t} \partial_{X}^{j} f-\sum_{m=0}^{i} \sum_{n=0}^{m} C_{m}^{n} \partial_{X}^{n} \partial_{t} X \cdot \partial_{X}^{m-n} \nabla \partial_{X}^{j-m-1} f
\end{aligned}
$$

which together with the inductive assumptions ensures that

$$
\begin{aligned}
& \left\|\partial_{X}^{i+1} \partial_{t} \partial_{X}^{j-i-1} X-\partial_{t} \partial_{X}^{j} X\right\|_{L_{t}^{s_{j}}\left(W^{1, p}\right)} \\
& \quad \lesssim \sum_{m=0}^{i} \sum_{n=0}^{m} C_{m}^{n}\left\|\partial_{X}^{n} \partial_{t} X\right\|_{L_{t}^{s_{j}}\left(W^{1, p}\right)}\left\|\partial_{X}^{m-n} \nabla \partial_{X}^{j-m-1} X\right\|_{L_{t}^{\infty}\left(W^{1, p}\right)} \lesssim J_{j}^{j+1} .
\end{aligned}
$$

(6.8) together with (6.9) and (6.10) shows that (6.3) holds for $\ell=j$. 
Exactly by the same manner, and using the fact that for any $\left.r_{j} \in\right] 1, \frac{2 k}{k+j \varepsilon}[$, there exist $\left.s_{n+1} \in\right] 2, \frac{2 k}{(n+1) \varepsilon}\left[\right.$ and $\left.s_{j-n-1} \in\right] 2, \frac{2 k}{(j-n-1) \varepsilon}[, n=0, \cdots, j-1$ such that

$$
\frac{1}{r_{j}}=\frac{1}{s_{n+1}}+\frac{1}{s_{j-n-1}}
$$

we can also prove that (6.4) and (6.5) holds for $\ell=j$. This completes the proof of Lemma 6.1 .

Before proceeding, let us take the operator $\partial_{X}^{\ell-1}$ to (5.10) to get

$$
\partial_{t} \partial_{X}^{\ell} v+v \cdot \nabla \partial_{X}^{\ell} v-(1+a)\left(\Delta \partial_{X}^{\ell} v-\nabla \partial_{X}^{\ell} \pi\right)=F_{\ell}(v, \pi), \quad \forall \ell=2, \cdots, k .
$$

where $F_{\ell}(v, \pi)$ can be inductively defined as

$$
F_{\ell}(v, \pi)=F_{1}\left(\partial_{X}^{\ell-1} v, \partial_{X}^{\ell-1} \pi\right)+\partial_{X} F_{\ell-1}(v, \pi), \quad \ell \geq 2 .
$$

Lemma 6.2. For $\ell=2, \cdots, k$ and for all $\left.r_{\ell} \in\right] 1, \frac{2 k}{k+\ell \varepsilon}[$, there holds

$$
\begin{aligned}
\left\|F_{\ell}\right\|_{L_{t}^{r_{\ell}}\left(L^{p}\right)} \lesssim J_{\ell-1}^{\ell+2}+J_{0}\left\|\partial_{X_{0}}^{\ell} a_{0}\right\|_{L_{t}^{\infty}\left(L^{\infty}\right)}+\left(\int _ { 0 } ^ { t } \left(\left\|\nabla v\left(t^{\prime}\right)\right\|_{L^{\infty}}^{r_{\ell}}\left\|\Delta \partial_{X}^{\ell-1} X\left(t^{\prime}\right)\right\|_{L^{p}}^{r_{\ell}}\right.\right. \\
\left.\left.+\left(\left\|\nabla^{2} v\left(t^{\prime}\right)\right\|_{L^{p}}^{r_{\ell}}+\left\|\nabla \pi\left(t^{\prime}\right)\right\|_{L^{p}}^{r_{\ell}}\right)\left\|\nabla \partial_{X}^{\ell-1} X\left(t^{\prime}\right)\right\|_{L^{\infty}}^{r_{\ell}}\right) d t^{\prime}\right)^{\frac{1}{r_{\ell}}} .
\end{aligned}
$$

Proof. In view of (6.12), one gets, by induction, that

$$
F_{\ell}(v, \pi)=F_{1}\left(\partial_{X}^{\ell-1} v, \partial_{X}^{\ell-1} \pi\right)+\partial_{X} F_{\ell-1}(v, \pi)=\sum_{i=0}^{\ell-1} \partial_{X}^{\ell-1-i} F_{1}\left(\partial_{X}^{i} v, \partial_{X}^{i} \pi\right),
$$

which along with the definition of $F_{1}$ given by (5.10), we write

$$
\begin{aligned}
F_{\ell}(v, \pi)=\sum_{i=0}^{\ell-1} \partial_{X}^{\ell-1-i}( & \partial_{X} a\left(\Delta \partial_{X}^{i} v-\nabla \partial_{X}^{i} \pi\right) \\
& \left.\quad-(1+a)\left(2 \nabla X: \nabla^{2} \partial_{X}^{i} v-\nabla X \cdot \nabla \partial_{X}^{i} \pi+\Delta X \cdot \nabla \partial_{X}^{i} v\right)\right) .
\end{aligned}
$$

Correspondingly $F_{\ell}$ reads

$$
\begin{aligned}
& F_{\ell}(v, \pi)=\sum_{i=0}^{\ell-1} \sum_{n=0}^{\ell-1-i} C_{\ell-1-i}^{n}( \partial_{X}^{n+1} a \partial_{X}^{\ell-1-i-n}\left(\Delta \partial_{X}^{i} v-\nabla \partial_{X}^{i} \pi\right) \\
&-2 \partial_{X}^{n}(1+a) \sum_{m=0}^{\ell-1-i-n} C_{\ell-1-i-n}^{m} \partial_{X}^{m} \nabla X: \partial_{X}^{\ell-1-i-n-m} \nabla^{2} \partial_{X}^{i} v \\
&+\partial_{X}^{n}(1+a) \sum_{m=0}^{\ell-1-i-n} C_{\ell-1-i-n}^{m} \partial_{X}^{m} \nabla X \cdot \partial_{X}^{\ell-1-i-n-m} \nabla \partial_{X}^{i} \pi \\
&\left.-\partial_{X}^{n}(1+a) \sum_{m=0}^{\ell-1-i-n} C_{\ell-1-i-n}^{m} \partial_{X}^{m} \Delta X \cdot \partial_{X}^{\ell-1-i-n-m} \nabla \partial_{X}^{i} v\right) \\
& \stackrel{\text { def }}{=} F_{\ell}^{1}+F_{\ell}^{2}+F_{\ell}^{3}+F_{\ell}^{4} .
\end{aligned}
$$


It is easy to observe that

$$
\begin{aligned}
F_{\ell}^{1}= & \sum_{i=1}^{\ell-1} \sum_{n=0}^{\ell-1-i} C_{\ell-1-i}^{j} \partial_{X}^{n+1} a \partial_{X}^{\ell-1-i-n}\left(\Delta \partial_{X}^{i} v-\nabla \partial_{X}^{i} \pi\right) \\
& +\sum_{n=0}^{\ell-2} C_{\ell-1}^{n} \partial_{X}^{n+1} a \partial_{X}^{\ell-1-n}(\Delta v-\nabla \pi)+\partial_{X}^{\ell} a(\Delta v-\nabla \pi),
\end{aligned}
$$

from which and Lemma 6.1, we infer

$$
\begin{aligned}
& \left\|F_{\ell}^{1}-\partial_{X}^{\ell} a(\Delta v-\nabla \pi)\right\|_{L_{t}^{r_{\ell-1}}\left(L^{p}\right)} \\
& \leq \sum_{i=1}^{\ell-1} \sum_{n=0}^{\ell-1-i} C_{\ell-1-i}^{n}\left\|\partial_{X}^{n+1} a\right\|_{L_{t}^{\infty}\left(L^{\infty}\right)}\left\|\partial_{X}^{\ell-1-i-n}\left(\Delta \partial_{X}^{i} v-\nabla \partial_{X}^{i} \pi\right)\right\|_{L_{t}^{r \ell-1}\left(L^{p}\right)} \\
& \quad+\sum_{n=0}^{\ell-2} C_{\ell-1}^{n}\left\|\partial_{X}^{n+1} a\right\|_{L_{t}^{\infty}\left(L^{\infty}\right)}\left\|\partial_{X}^{\ell-1-n}(\Delta v-\nabla \pi)\right\|_{L_{t}^{r_{\ell-1}}\left(L^{p}\right)} \lesssim J_{\ell-1}^{\ell+2} .
\end{aligned}
$$

By the same manner, we also have

$$
\begin{array}{r}
\left\|F_{\ell}^{2}+2(1+a) \partial_{X}^{\ell-1} \nabla X: \nabla^{2} v\right\|_{L_{t}^{r_{\ell-1}\left(L^{p}\right)}}+\left\|F_{\ell}^{3}-(1+a) \partial_{X}^{\ell-1} \nabla X \cdot \nabla \pi\right\|_{L_{t}^{r_{\ell-1}}\left(L^{p}\right)} \\
+\left\|F_{\ell}^{4}+(1+a) \partial_{X}^{\ell-1} \Delta X \cdot \nabla v\right\|_{L_{t}^{r_{\ell-1}}\left(L^{p}\right)} \lesssim J_{\ell-1}^{\ell+2} .
\end{array}
$$

As a consequence, we conclude

$$
\begin{aligned}
\| F_{\ell}(v, \pi)-\partial_{X}^{\ell} a(\Delta v & -\nabla \pi)+(1+a)\left(2 \partial_{X}^{\ell-1} \nabla X: \nabla^{2} v\right. \\
& \left.-\partial_{X}^{\ell-1} \nabla X \cdot \nabla \pi+\partial_{X}^{\ell-1} \Delta X \cdot \nabla v\right) \|_{L_{t}^{r_{\ell-1}\left(L^{p}\right)}} \lesssim J_{\ell-1}^{\ell+2},
\end{aligned}
$$

which ensures (6.13) thanks to (4.14), (5.7) and (6.3). This completes the proof of Lemma 6.2 .

We also need the following estimate for the pressure function.

Lemma 6.3. For $\ell=2, \cdots, k$ and $\left.r_{\ell} \in\right] 1, \frac{2 k}{k+\ell \varepsilon}[$, one has

$$
\begin{aligned}
& \left\|\nabla \partial_{X}^{\ell} \pi\right\|_{L_{t}^{r_{\ell}\left(L^{p}\right)}} \lesssim J_{\ell-1}^{\ell+2}+J_{0}\left\|\partial_{X_{0}}^{\ell} a_{0}\right\|_{L_{t}^{\infty}\left(L^{\infty}\right)}+\left\|a_{0}\right\|_{L^{\infty}}\left\|\Delta \partial_{X}^{\ell} v\right\|_{L_{t}^{r}\left(_{\left(L^{p}\right)}\right.} \\
& +\left(\int_{0}^{t}\left\|a_{0}\right\|_{L^{\infty}}\left\|\nabla v\left(t^{\prime}\right)\right\|_{L^{\infty}}^{r_{\ell}}\left\|\Delta \partial_{X}^{\ell-1} X\left(t^{\prime}\right)\right\|_{L^{p}}\right. \\
& \left.+\left\|\left(v \cdot \nabla v\left(t^{\prime}\right), \Delta v\left(t^{\prime}\right), \nabla \pi\left(t^{\prime}\right)\right)\right\|_{L^{p}}^{r_{\ell}}\left\|\nabla \partial_{X}^{\ell-1} X\left(t^{\prime}\right)\right\|_{L^{\infty}}^{r_{\ell}} d t^{\prime}\right)^{\frac{1}{r_{\ell}}} .
\end{aligned}
$$

Proof. Motivated by (4.17) and (5.11), we assume that $\partial_{X}^{\ell-1} \pi$ verifies

$$
\operatorname{div}\left((1+a) \nabla \partial_{X}^{\ell-1} \pi\right)=\operatorname{div} G_{\ell-1} .
$$

As $\operatorname{div} X=0$, we have

$$
\partial_{X} \operatorname{div} f=\operatorname{div}\left(\partial_{X} f\right)-\operatorname{div}(f \cdot \nabla X),
$$

so that we have

$$
\begin{aligned}
\operatorname{div}\left((1+a) \nabla \partial_{X}^{\ell} \pi\right)=\operatorname{div}( & \partial_{X} G_{\ell-1}-G_{\ell-1} \cdot \nabla X-\partial_{X} a \nabla \partial_{X}^{\ell-1} \pi \\
& \left.+(1+a)\left(\nabla \partial_{X}^{\ell-1} \pi \cdot \nabla X+\nabla X \cdot \nabla \partial_{X}^{\ell-1} \pi\right)\right) \stackrel{\text { def }}{=} \operatorname{div} G_{\ell} .
\end{aligned}
$$


Let us assume by induction that for $0 \leq i \leq j \leq \ell-1$,

$$
\left\|\partial_{X}^{i} G_{\ell-1-j}\right\|_{L_{t}^{r} \ell-1\left(L^{p}\right)} \lesssim J_{\ell-1}^{\ell+1+i-j} .
$$

It is easy to observe from (6.16) that

$$
\begin{aligned}
\partial_{X}^{i} G_{\ell-j}=\partial_{X}^{i}( & \partial_{X} G_{\ell-1-j}-G_{\ell-1-j} \cdot \nabla X-\partial_{X} a \nabla \partial_{X}^{\ell-1-j} \pi \\
& \left.+(1+a)\left(\nabla \partial_{X}^{\ell-1-j} \pi \cdot \nabla X+\nabla X \cdot \nabla \partial_{X}^{\ell-1-j} \pi\right)\right) .
\end{aligned}
$$

We shall decompose the estimate of $\partial_{X}^{i} G_{\ell-j}$ with $0 \leq i \leq j \leq \ell$ into the following cases:

(1) $0 \leq i<j \leq \ell-2$. It follows from the inductive assumption (6.17) and Remark 6.1 that for $0 \leq i \leq j \leq \ell-2$,

$$
\begin{aligned}
&\left\|\partial_{X}^{i+1} G_{\ell-1-j}\right\|_{L_{t}^{r \ell-1}\left(L^{p}\right)} \lesssim J_{\ell-1}^{\ell+2+i-j}, \text { if moreover } i<j \\
&\left\|\partial_{X}^{i}\left(G_{\ell-1-j} \cdot \nabla X\right)\right\|_{L_{t}^{r \ell-1}\left(L^{p}\right)} \lesssim \sum_{0 \leq m \leq i}\left\|\partial_{X}^{m}\left(G_{\ell-1-j}\right)\right\|_{L_{t}^{r \ell-1}\left(L^{p}\right)}\left\|\partial_{X}^{i-m} \nabla X\right\|_{L_{t}^{\infty}\left(L^{\infty}\right)} \\
& \lesssim J_{\ell-1}^{\ell+1+m-j} J_{i-m+1}^{i-m+1} \lesssim J_{\ell-1}^{\ell+2+i-j}, \\
&\left\|\partial_{X}^{i}\left(\partial_{X} a \nabla \partial_{X}^{\ell-1-j} \pi\right)\right\|_{L_{t}^{r \ell-1}\left(L^{p}\right)} \lesssim \sum_{0 \leq m \leq i}\left\|\partial_{X}^{m+1} a\right\|_{L_{t}^{\infty}\left(L^{\infty}\right)}\left\|\partial_{X}^{i-m} \nabla \partial_{X}^{\ell-1-j} \pi\right\|_{L_{t}^{r \ell-1}\left(L^{p}\right)} \\
& \lesssim J_{m+1} J_{\ell-1+i-m-j}^{\ell+i-m-j} \lesssim J_{\ell-1}^{\ell+1+i-j},
\end{aligned}
$$

and

$$
\begin{aligned}
& \left\|\partial_{X}^{i}\left((1+a) \nabla \partial_{X}^{\ell-1-j} \pi \otimes \nabla X\right)\right\|_{L_{t}^{r_{\ell-1}\left(L^{p}\right)}} \\
& \lesssim \sum_{\substack{0 \leq m \leq i \\
0 \leq n \leq i-m}}\left\|\partial_{X}^{m}(1+a)\right\|_{L_{t}^{\infty}\left(L^{\infty}\right)}\left\|\partial_{X}^{n} \nabla \partial_{X}^{\ell-1-j} \pi\right\|_{L_{t}^{r \ell-1}\left(L^{p}\right)}\left\|\partial_{X}^{i-m-n} \nabla X\right\|_{L_{t}^{\infty}\left(L^{\infty}\right)} \\
& \lesssim J_{m} J_{\ell-1+n-j}^{\ell+n-j} J_{i+1-m-n}^{i+1-m-n} \lesssim J_{\ell-1}^{\ell+2+i-j} .
\end{aligned}
$$

Hence we obtain

$$
\left.\left\|\partial_{X}^{i} G_{\ell-j}\right\|_{L_{t}^{r} \ell-1} L^{p}\right) \lesssim J_{\ell-1}^{\ell+2+i-j} \quad \text { when } \quad 0 \leq i<j \leq \ell-2 .
$$

(2) $0 \leq i=j \leq \ell-2$. We first deduce from case (1) that

$$
\partial_{X}^{i} G_{\ell-i}=\partial_{X}^{i+1} G_{\ell-1-i}+R_{\ell}^{\ell-i} \quad \text { with } \quad\left\|R_{\ell}^{\ell-i}\right\|_{L_{t}^{r \ell-1}\left(L^{p}\right)} \lesssim J_{\ell-1}^{\ell+2} .
$$

Along this line, we can show that

$$
\partial_{X}^{i} G_{\ell-i}=\partial_{X}^{\ell-1} G_{1}+R_{\ell}^{2} \quad \text { with } \quad\left\|R_{\ell}^{2}\right\|_{L_{t}^{r \ell-1}\left(L^{p}\right)} \lesssim J_{\ell-1}^{\ell+2}
$$

This shows that

$$
\left.\left\|\partial_{X}^{i} G_{\ell-i}-\partial_{X}^{\ell-1} G_{1}\right\|_{L_{t}^{r} \ell-1} L^{p}\right) \lesssim J_{\ell-1}^{\ell+2} \quad \text { for } \quad i=0, \cdots \ell-2
$$

with

$$
\begin{aligned}
& G_{1}=\partial_{X} G_{0}-G_{0} \cdot \nabla X-\partial_{X} a \nabla \pi+(1+a)(\nabla \pi \cdot \nabla X+\nabla X \cdot \nabla \pi), \\
& G_{0}=-v \cdot \nabla v+a \Delta v .
\end{aligned}
$$


Noticing that

$$
\begin{aligned}
& \|\left(\partial_{X}^{\ell-1} G_{1}-\partial_{X}^{\ell} G_{0}+G_{0} \cdot \partial_{X}^{\ell-1} \nabla X+\partial_{X}^{\ell} a \nabla \pi\right. \\
& \left.\quad-(1+a)\left(\nabla \pi \cdot \partial_{X}^{\ell-1} \nabla X+\partial_{X}^{\ell-1} \nabla X \cdot \nabla \pi\right)\right) \|_{L_{t}^{r_{\ell-1}}\left(L^{p}\right)} \lesssim J_{\ell-1}^{\ell+2}, \\
& \left\|\partial_{X}^{\ell} G_{0}+\partial_{X}^{\ell} v \cdot \nabla v+v \cdot \partial_{X}^{\ell} \nabla v-\partial_{X}^{\ell} a \Delta v-a \partial_{X}^{\ell} \Delta v\right\|_{L_{t}^{r \ell-1}\left(L^{p}\right)} \lesssim J_{\ell-1}^{\ell+2},
\end{aligned}
$$

and

$$
\begin{array}{r}
\left\|v \cdot \nabla \partial_{X}^{\ell} v\right\|_{L_{t}^{r \ell}\left(L^{p}\right)} \lesssim\|v\|_{L_{t}^{s}\left(L^{\infty}\right)}\left(\|\nabla X\|_{L_{t}^{\infty}\left(L^{p}\right)}\left\|\nabla \partial_{X}^{\ell-1} v\right\|_{L_{t}^{r_{\ell-1}\left(L^{\infty}\right)}}\right. \\
+\|X\|_{L_{t}^{\infty}\left(L^{\infty}\right)}\left\|\Delta \partial_{X}^{\ell-1} v\right\|_{L_{t}^{r} \ell-1}\left(L^{p}\right) \\
\left\|\partial_{X}^{\ell} v \cdot \nabla v\right\|_{L_{t}^{r \ell}\left(L^{p}\right)} \leq\|X\|_{L_{t}^{\infty}\left(L^{\infty}\right)}\left\|\nabla \partial_{X}^{\ell-1} v\right\|_{L_{t}^{r \ell-1}\left(L^{\infty}\right)}\|\nabla v\|_{L_{t}^{s}\left(L^{p}\right)},
\end{array}
$$

where $s \in] 2, \infty\left[\right.$ so that $\frac{1}{r_{\ell}}=\frac{1}{s}+\frac{1}{r_{\ell-1}}$, we know that

$$
\left\|\partial_{X}^{i} G_{\ell-i}\right\|_{L_{t}^{r}\left(L^{p}\right)} \lesssim J_{\ell}^{\ell+2}
$$

(3) $j=\ell-1,0 \leq i \leq \ell-2$. Note that when $j=\ell-1$,

$$
G_{\ell-1-j}=G_{0}=-v \cdot \nabla v+a \Delta v, \quad \nabla \partial_{X}^{\ell-1-j} \pi=\nabla \pi
$$

by the same arguments as in the case $0 \leq i<j \leq \ell-2$ one obtains also the estimate $\left.\left\|\partial_{X}^{i} G_{\ell-j}\right\|_{L_{t}^{r} \ell-1} L^{p}\right) \lesssim J_{\ell-1}^{\ell+2+i-j}$ for the case $0 \leq i \leq \ell-2, j=\ell-1$.

(4) $j=\ell, 0 \leq i \leq \ell-1$. In this case, there holds

$$
\left\|\partial_{X}^{i} G_{0}\right\|_{L_{t}^{r}{ }^{r-1}\left(L^{p}\right)} \lesssim J_{\ell-1}^{i+2} .
$$

(5) $i=j=\ell-1$ or $i=j=\ell$. As in the case when $0 \leq i=j \leq \ell-2$, one has

$$
\left\|\partial_{X}^{\ell-1} G_{1}\right\|_{L_{t}^{r \ell}\left(L^{p}\right)},\left\|\partial_{X}^{\ell} G_{0}\right\|_{L_{t}^{r \ell}\left(L^{p}\right)} \lesssim J_{\ell}^{\ell+2} .
$$

To conclude, we have arrived at the following for $0 \leq i \leq j \leq \ell \leq k$ :

$$
\left\|\partial_{X}^{i} G_{\ell-j}\right\|_{L_{t}^{r \ell}\left(L^{p}\right)} \lesssim J_{\ell}^{\ell+2+i-j}
$$

and hence (6.17) follows by induction.

Thanks to (6.17), (6.18) and (6.19), we infer

$$
\begin{aligned}
\| G_{\ell}-\partial_{X}^{\ell} a \Delta v-a \partial_{X}^{\ell} \Delta v & +G_{0} \cdot \partial_{X}^{\ell-1} \nabla X+\partial_{X}^{\ell} a \nabla \pi \\
& -(1+a)\left(\nabla \pi \cdot \partial_{X}^{\ell-1} \nabla X+\partial_{X}^{\ell-1} \nabla X \cdot \nabla \pi\right) \|_{L_{t}^{r_{\ell-1}\left(L^{p}\right)}} \lesssim J_{\ell-1}^{\ell+2} .
\end{aligned}
$$

We apply (6.5) to deal with the term $\left[\partial_{X}^{\ell} \Delta v ; \Delta \partial_{X}^{\ell} v\right]$ and (6.3) to the term $\left[\partial_{X}^{\ell-1} \nabla X ; \nabla \partial_{X}^{\ell-1} X\right]$. Then (6.15) follows from (6.16) and (6.20).

We now turn to the proof of Proposition 6.1.

Proof of Proposition 6.1. - The estimates of $\partial_{t} \partial_{X}^{\ell} v, \Delta \partial_{X}^{\ell} v$ and $\nabla \partial_{X}^{\ell} \pi$

As in the previous sections, we reformulate (6.11) as

$$
\begin{gathered}
\partial_{X}^{\ell} v(t)=e^{t \Delta} \partial_{X_{0}}^{\ell} v_{0}+\int_{0}^{t} e^{\left(t-t^{\prime}\right) \Delta} \tilde{F}_{\ell}\left(t^{\prime}\right) d t^{\prime} \quad \text { with } \\
\tilde{F}_{\ell} \stackrel{\text { def }}{=}-v \cdot \nabla \partial_{X}^{\ell} v+a \Delta \partial_{X}^{\ell} v-(1+a) \nabla \partial_{X}^{\ell} \pi+F_{\ell}(v, \pi),
\end{gathered}
$$


from which and (5.2), we infer

$$
\left\|\partial_{t} \partial_{X}^{\ell} v\right\|_{L_{t}^{r \ell}\left(L^{p}\right)}+\left\|\Delta \partial_{X}^{\ell} v\right\|_{L_{t}^{r_{\ell}\left(L^{p}\right)}} \leq C\left(\left\|\partial_{X_{0}}^{\ell} v_{0}\right\|_{W^{1-\frac{\ell}{k} \varepsilon, p}}+\left\|\tilde{F}_{\ell}\right\|_{L_{t}^{r_{\ell}}\left(L^{p}\right)}\right) .
$$

Note that for any $\left.r_{\ell} \in\right] 1, \frac{2 k}{k+\ell \varepsilon}\left[\right.$, there exist $\left.\sigma_{1}, \sigma_{2} \in\right] 2, \infty\left[\right.$ and $\left.r_{\ell-1} \in\right] 1, \frac{2 k}{k+(\ell-1) \varepsilon}\left[, s_{\ell-1} \in\right.$ ] $2, \frac{2 k}{(\ell-1) \varepsilon}\left[\right.$ so that $\frac{1}{r_{\ell}}=\frac{1}{\sigma_{1}}+\frac{1}{s_{\ell-1}}=\frac{1}{\sigma_{2}}+\frac{1}{r_{\ell-1}}$. Then it follows from Remark 6.1 that

$$
\begin{aligned}
\left\|v \cdot \nabla \partial_{X}^{\ell} v\right\|_{L_{t}^{r_{\ell}\left(L^{p}\right)}} \lesssim & \|v\|_{L_{t}^{\sigma_{1}\left(L^{\infty}\right)}}\|\nabla X\|_{L_{t}^{\infty}\left(L^{\infty}\right)}\left\|\nabla \partial_{X}^{\ell-1} v\right\|_{L_{t}^{s_{\ell-1}\left(L^{p}\right)}} \\
& +\|v\|_{L_{t}^{\sigma_{2}\left(L^{\infty}\right)}}\|X\|_{L_{t}^{\infty}\left(L^{\infty}\right)}\left\|\nabla^{2} \partial_{X}^{\ell-1} v\right\|_{L_{t}^{r_{\ell-1}\left(L^{p}\right)}} \lesssim J_{\ell-1}^{\ell+2} .
\end{aligned}
$$

Resuming the above estimate, the Estimates (6.13) and (6.15) into (6.22) and taking $\left\|a_{0}\right\|_{L^{\infty}}$ to be sufficiently small, we deduce from the maximal regularity estimate to the heat semigroup that

$$
\begin{array}{r}
\left\|\partial_{t} \partial_{X}^{\ell} v\right\|_{L_{t}^{r_{\ell}\left(L^{p}\right)}}+\left\|\Delta \partial_{X}^{\ell} v\right\|_{L_{t}^{r_{\ell}\left(L^{p}\right)}}+\left\|\nabla \partial_{X}^{\ell} \pi\right\|_{L_{t}^{r_{\ell}\left(L^{p}\right)}} \leq \mathcal{H}_{\ell}(t)+C\left(\int _ { 0 } ^ { t } \left(\left\|\nabla^{2} v\left(t^{\prime}\right)\right\|_{L^{p}}^{r_{\ell}}\right.\right. \\
\left.\left.+\left\|\nabla \pi\left(t^{\prime}\right)\right\|_{L^{p}}^{r_{\ell}}+\left(1+\left\|v\left(t^{\prime}\right)\right\|_{L^{p}}\right)^{r_{\ell}}\left\|\nabla v\left(t^{\prime}\right)\right\|_{L^{\infty}}^{r_{\ell}}\right)\left\|\nabla \partial_{X}^{\ell-1} X\left(t^{\prime}\right)\right\|_{W^{1, p}}^{r_{\ell}} d t^{\prime}\right)^{\frac{1}{r_{\ell}}} .
\end{array}
$$

- The estimates of $\Delta \partial_{X}^{\ell-1} X$

We first get, by applying the operator $\partial_{X}^{\ell-1}$ to (3.6), that

$$
\partial_{t} \partial_{X}^{\ell-1} X+v \cdot \nabla \partial_{X}^{\ell-1} X=\partial_{X}^{\ell} v
$$

from which, we deduce, by using $L^{p}$ type energy estimate and (5.5), that

$$
\begin{aligned}
\left\|\partial_{X}^{\ell-1} X(t)\right\|_{L^{p}} & \leq\left\|\partial_{X_{0}}^{\ell-1} X_{0}\right\|_{L^{p}}+\left\|\partial_{X}^{\ell} v\right\|_{L_{t}^{1}\left(L^{p}\right)} \\
& \leq\left\|\partial_{X_{0}}^{\ell-1} X_{0}\right\|_{L^{p}}+\|X\|_{L_{t}^{\infty}\left(L^{\infty}\right)}\left\|\nabla \partial_{X}^{\ell-1} v\right\|_{L_{t}^{1}\left(L^{p}\right)} \leq J_{\ell-1}^{\ell+1},
\end{aligned}
$$

and

$$
\begin{aligned}
\left\|\nabla \partial_{X}^{\ell-1} X(t)\right\|_{L^{p}} \leq & \left\|\nabla \partial_{X_{0}}^{\ell-1} X_{0}\right\|_{L^{p}}+\int_{0}^{t}\left(\left\|\nabla v\left(t^{\prime}\right)\right\|_{L^{\infty}}\left\|\nabla \partial_{X}^{\ell-1} X\left(t^{\prime}\right)\right\|_{L^{p}}\right. \\
& \left.+\left\|\nabla X\left(t^{\prime}\right)\right\|_{L^{\infty}}\left\|\nabla \partial_{X}^{\ell-1} v\left(t^{\prime}\right)\right\|_{L^{p}}+\left\|X\left(t^{\prime}\right)\right\|_{L^{\infty}}\left\|\nabla^{2} \partial_{X}^{\ell-1} v\left(t^{\prime}\right)\right\|_{L^{p}}\right) d t^{\prime} .
\end{aligned}
$$

Applying Gronwall's inequality and using (5.5), we obtain

$$
\begin{aligned}
\left\|\nabla \partial_{X}^{\ell-1} X\right\|_{L_{t}^{\infty}\left(L^{p}\right)} \leq & \left(\left\|\nabla \partial_{X_{0}}^{\ell-1} X_{0}\right\|_{L^{p}}+\|\nabla X\|_{L_{t}^{\infty}\left(L^{\infty}\right)}\left\|\nabla \partial_{X}^{\ell-1} v\right\|_{L_{t}^{1}\left(L^{p}\right)}\right. \\
& \left.+\|X\|_{L_{t}^{\infty}\left(L^{\infty}\right)}\left\|\nabla^{2} \partial_{X}^{\ell-1} v\right\|_{L_{t}^{1}\left(L^{p}\right)}\right) \exp \left(\|\nabla v\|_{L_{t}^{1}\left(L^{\infty}\right)}\right) \lesssim J_{\ell-1}^{\ell+1} \exp \left(C J_{0}\right) .
\end{aligned}
$$

This gives

$$
\left\|\partial_{X}^{\ell-1} X\right\|_{L_{t}^{\infty}\left(W^{1, p}\right)} \leq \mathcal{H}_{\ell}(t) .
$$

By the same manner, noting that

$$
\partial_{t} \Delta \partial_{X}^{\ell-1} X+v \cdot \nabla \Delta \partial_{X}^{\ell-1} X=-\Delta v \cdot \nabla \partial_{X}^{\ell-1} X-2 \nabla v: \nabla^{2} \partial_{X}^{\ell-1} X+\Delta \partial_{X}^{\ell} v,
$$

we get, by using $L^{p}$ type energy estimate, that

$$
\begin{aligned}
\left\|\Delta \partial_{X}^{\ell-1} X(t)\right\|_{L^{p}} \leq & \left\|\Delta \partial_{X_{0}}^{\ell-1} X_{0}\right\|_{L^{p}}+\int_{0}^{t}\left(\left\|\Delta v\left(t^{\prime}\right)\right\|_{L^{p}}\left\|\nabla \partial_{X}^{\ell-1} X\left(t^{\prime}\right)\right\|_{L^{\infty}}\right. \\
& \left.+2\left\|\nabla v\left(t^{\prime}\right)\right\|_{L^{\infty}}\left\|\nabla^{2} \partial_{X}^{\ell-1} X\left(t^{\prime}\right)\right\|_{L^{p}}+\left\|\Delta \partial_{X}^{\ell} v\left(t^{\prime}\right)\right\|_{L^{p}}\right) d t^{\prime} .
\end{aligned}
$$


Using (5.8) and then applying Gronwall's inequality, we achieve

$$
\begin{aligned}
\left\|\Delta \partial_{X}^{\ell-1} X\right\|_{L_{t}^{\infty}\left(L^{p}\right)} \leq & \left(\left\|\Delta \partial_{X_{0}}^{\ell-1} X_{0}\right\|_{L^{p}}+\left\|\nabla \partial_{X}^{\ell-1} X\right\|_{L_{t}^{\infty}\left(L^{p}\right)}\|\Delta v\|_{L_{t}^{1}\left(L^{p}\right)}+\left\|\Delta \partial_{X}^{\ell} v\right\|_{L_{t}^{1}\left(L^{p}\right)}\right) \\
& \times \exp \left(C\left(\|\nabla v\|_{L_{t}^{1}\left(L^{\infty}\right)}+\|\Delta v\|_{L_{t}^{1}\left(L^{p}\right)}\right)\right) \\
\leq & \mathcal{H}_{\ell}(t)\left(1+\left\|\Delta \partial_{X}^{\ell} v\right\|_{L_{t}^{1}\left(L^{p}\right)}\right) .
\end{aligned}
$$

Substituting (6.23) into (6.25) gives rise to

$$
\begin{aligned}
\left\|\Delta \partial_{X}^{\ell-1} X(t)\right\|_{L^{p}} \leq \mathcal{H}_{\ell}(t)+\mathcal{H}_{\ell}(t) & \left(\int _ { 0 } ^ { t } \left(\left\|\nabla^{2} v\left(t^{\prime}\right)\right\|_{L^{p}}^{r_{\ell}}+\left\|\nabla \pi\left(t^{\prime}\right)\right\|_{L^{p}}^{r_{\ell}}\right.\right. \\
& \left.\left.+\left(1+\left\|v\left(t^{\prime}\right)\right\|_{L^{p}}^{r_{\ell}}\right)\left\|\nabla v\left(t^{\prime}\right)\right\|_{L^{\infty}}^{r_{\ell}}\right)\left\|\Delta \partial_{X}^{\ell-1} X\left(t^{\prime}\right)\right\|_{L^{p}}^{r_{\ell}} d t^{\prime}\right)^{\frac{1}{r_{\ell}}} .
\end{aligned}
$$

Taking $r_{\ell}$ th power to the above inequality and then applying Gronwall's inequality yields

$$
\left\|\Delta \partial_{X}^{\ell-1} X\right\|_{L_{t}^{\infty}\left(L^{p}\right)} \leq \mathcal{H}_{\ell}(t),
$$

which together with (6.23) and (6.24) ensures that

$$
\left\|\partial_{t} \partial_{X}^{\ell} v\right\|_{L_{t}^{r} \ell\left(L^{p}\right)}+\left\|\Delta \partial_{X}^{\ell} v\right\|_{L_{t}^{r \ell}\left(L^{p}\right)}+\left\|\nabla \partial_{X}^{\ell} \pi\right\|_{L_{t}^{r \ell}\left(L^{p}\right)}+\left\|\partial_{X}^{\ell-1} X\right\|_{L_{t}^{\infty}\left(W^{2, p}\right)} \leq \mathcal{H}_{\ell}(t) .
$$

Correspondingly for $s_{\ell}$ determined by $\frac{1}{r_{\ell}}=\frac{1}{2}+\frac{1}{s_{\ell}}$, we have

$$
\left\|\nabla \partial_{X}^{\ell} v\right\|_{L_{t}^{r \ell}\left(L^{\infty}\right) \cap L_{t}^{s \ell}\left(L^{p}\right)}+\left\|\partial_{X}^{\ell} v\right\|_{L_{t}^{s \ell}\left(L^{\infty}\right)}+\left\|\partial_{t} \partial_{X}^{\ell-1} X\right\|_{L_{t}^{s \ell}\left(W^{1, p}\right)} \leq \mathcal{H}_{\ell}(t) .
$$

This completes the proof of Proposition 6.1.

Finally we are in a position to complete the proof of Theorem 3.1.

Proof of Theorem 3.1. In order to complete the proof of Theorem 3.1, we first mollify the initial data to be $\left(\rho_{0, n}, v_{0, n}, X_{0, n}\right)$ so that according to [22], (1.1) has a unique global solution $\left(\rho_{n}, v_{n}, X_{n}\right)$ and then repeating the proofs of Proposition 4.2, Proposition 5.1 and Proposition 6.1 to show that the approximate solutions $\left(\rho_{n}, v_{n}, X_{n}\right)$ satisfies the uniform estimates (4.14), (5.5) and (6.2). Finally a standard compactness argument as that in [11] can be applied to prove the limit $(\rho, v, X)$ of such approximate solutions satisfies the required estimate of Theorem 3.1. Since the velocity field $v \in L_{\text {loc }}^{1}\left(\mathbb{R}^{+} ;\right.$Lip), the uniqueness of such solutions can be proved by using Lagrangian formulation of (1.1) as that in $[14,19]$. We omit the details here.

\section{The PRoOF of (3.15)}

The goal of this section is to present the proof of (3.15). Similar to Section 2.2 of [8], we define the product operator $R_{q}$ as follows

$$
R_{q}\left(\alpha_{1}, \cdots, \alpha_{m}\right) \stackrel{\text { def }}{=} \int_{[0,1]^{m}} \int_{\mathbb{R}^{2}} \prod_{j=1}^{m} \alpha_{j}\left(x+f_{j}(\tau) 2^{-q} y\right) h(\tau, y) d y d \tau,
$$

where $h \in C\left([0,1]^{m} \times \mathbb{R}^{2} ; \mathcal{S}\left(\mathbb{R}^{2}\right)\right), f_{j} \in L^{\infty}\left([0,1]^{m}\right)$ and $f_{j}(\tau) \neq 0$ for $\left.\tau \in\right] 0,1\left[{ }^{m}\right.$, and there exists a nonnegative function $H(t, r) \in C\left([0,1]^{m} ; L^{1}\right)$, which is non-increasing with respect to $r$ variable so that

$$
|h(t, y)| \leq H(t,|y|) .
$$


We also recall the definition of the maximal function to a $L_{\mathrm{loc}}^{1}$ function $f$ from [5]

$$
\forall x \in \mathbb{R}^{2}, \quad M f(x) \stackrel{\text { def }}{=} \sup _{r>0} \frac{1}{\pi r^{2}} \int_{|y-x| \leq r}|f(y)| d y .
$$

Lemma 7.1. Let $X$ be a $\delta, k$-regular vector field (see (3.3)) and $\alpha_{j}$ satisfy supp $\widehat{\alpha}_{j} \subset B\left(0,2^{q}\right)$, for $j=1, \cdots, m$. Then for any $\ell \leq k$, there exist integers $\gamma_{n}\left(\lambda_{i}\right)$ such that

$$
\begin{aligned}
\mid\left(T_{X \cdot \nabla}\right)^{\ell} & R_{q}\left(\alpha_{1}, \cdots, \alpha_{m}\right) \mid \\
& \lesssim \sum_{n,|\lambda| \leq \ell} 2^{q(\ell-|\lambda|) \delta} \min _{i=1, \cdots, m}\left\{M^{\gamma_{n}\left(\lambda_{i}\right)}\left(\left(T_{X \cdot \nabla}\right)^{\lambda_{i}} \alpha_{i}\right) \prod_{j=1, j \neq i}^{m}\left\|\left(T_{X \cdot \nabla}\right)^{\lambda_{j}} \alpha_{j}\right\|_{L^{\infty}}\right\} .
\end{aligned}
$$

Here and in what follows, $A \lesssim B$ means that there exists a positive constant $C$ which depends on $\|X\|_{\delta, X}^{1-\delta, k-1}$ so that $A \leq C B$.

With the above lemma, similar to Theorem 2.2.1 of [8], Theorem 3.2.2 of [9] and Lemma 2.2 of [25], we have

Lemma 7.2. If the vector field $X$ is $\delta, k$-regular, let the striated norms $\|\cdot\|_{\delta, X}^{\sigma, \ell},\left.|\cdot| \cdot\right|_{\delta, X} ^{s, p, \ell}$ and $\mid \cdot \|_{\delta, X}^{s, p, \ell}$ be given respectively by (3.2), (3.4) and (3.5). Then the following statements hold for all $\ell \leq k$ :

(I) if supp $\widehat{v}$, supp $\widehat{w} \subset B\left(0,2^{q}\right)$, then there exist integers $\gamma_{n}\left(\lambda_{1}\right)$, so that

$$
\left|\left(T_{X \cdot \nabla}\right)^{\ell}(v w)\right| \lesssim \sum_{n, \lambda_{1}+\lambda_{2} \leq \ell} 2^{q\left(\ell-\lambda_{1}-\lambda_{2}\right) \delta} M^{\gamma_{n}\left(\lambda_{1}\right)}\left(\left(T_{X \cdot \nabla}\right)^{\lambda_{1}} v\right)\left\|\left(T_{X \cdot \nabla}\right)^{\lambda_{2}} w\right\|_{L^{\infty}} ;
$$

(II) for any smooth function $\theta$ with compact support in an annulus $\mathcal{C}$, one has

$$
\begin{aligned}
\left\|\left(T_{X \cdot \nabla}\right)^{\ell} \theta\left(2^{-q} D\right) w\right\|_{L^{\infty}} & \lesssim 2^{q(\ell \delta-\sigma)}\|w\|_{\delta, X}^{\sigma, \ell}, \\
\left\|\left(T_{X \cdot \nabla}\right)^{\ell} \theta\left(2^{-q} D\right) w\right\|_{L^{\infty}} & \lesssim \sum_{0 \leq \lambda \leq \ell} 2^{q(\ell-\lambda) \delta}\left\|\left(T_{X \cdot \nabla}\right)^{\lambda} w\right\|_{L^{\infty}} ;
\end{aligned}
$$

and there exist integers $\gamma_{n}(\lambda)$ so that

$$
\begin{aligned}
& \left\|\left(2^{q(s-\ell \delta)}\left(T_{X \cdot \nabla}\right)^{\ell} \theta\left(2^{-q} D\right) w\right)_{\ell^{2}(\mathbb{N})}\right\|_{L^{p}} \lesssim|w|_{\delta, X}^{s, p, \ell}, \\
& \left|\left(T_{X \cdot \nabla}\right)^{\ell} \theta\left(2^{-q} D\right) w\right| \lesssim \sum_{0 \leq n, \lambda \leq \ell} 2^{q(\ell-\lambda) \delta} M^{\gamma_{n}(\lambda)}\left(\left(T_{X \cdot \nabla}\right)^{\lambda} w\right) ;
\end{aligned}
$$

(III) let $\chi(\xi)$ be smooth $S^{m}$ Fourier-multiplier with $\chi(L \xi)=L^{m} \chi(\xi)$ for $|\xi| \geq M$, then

$$
\|\chi(D) w\|_{\delta, X}^{\sigma-m, \ell} \lesssim\|w\|_{\delta, X}^{\sigma, \ell}, \quad \text { and } \quad|\chi(D) w|_{\delta, X}^{s-m, p, \ell} \lesssim|w|_{\delta, X}^{s, p, \ell}
$$

(IV) We also have the following estimates for the paraproduct and the remainder term to the Bony's decomposition:

$$
\left\|T_{v} w\right\|_{\delta, X}^{\sigma_{1}+\sigma_{2}, \ell} \lesssim\|v\|_{\delta, X}^{\sigma_{1}, \ell}\|w\|_{\delta, X}^{\sigma_{2}, \ell} \text { if } \sigma_{1}<0, \quad\left\|T_{v} w\right\|_{\delta, X}^{\sigma_{2}, \ell} \lesssim\left(\|v\|_{L^{\infty}}+\|v\|_{\delta, X}^{0, \ell}\right)\|w\|_{\delta, X}^{\sigma_{2}, \ell},
$$

and

$$
\left|T_{v} w\right|_{\delta, X}^{s+\sigma, p, \ell} \lesssim\|v\|_{\delta, X}^{\sigma, \ell}|w|_{\delta, X}^{s, p, \ell} \text { if } \sigma<0, \quad\left|T_{v} w\right|_{\delta, X}^{s, p, \ell} \lesssim\left(\|v\|_{L^{\infty}}+\|v\|_{\delta, X}^{0, \ell}\right)|w|_{\delta, X}^{s, p, \ell},
$$

or for any $\epsilon>0$,

$$
\left|T_{v} w\right|_{\delta, X}^{\sigma+s, p, \ell} \lesssim|v|_{\delta, X}^{s, p, \ell}\|w\|_{\delta, X}^{\sigma, \ell} \quad \text { if } s<0, \quad\left|T_{v} w\right|_{\delta, X}^{\sigma, p, \ell} \lesssim\left(\|v\|_{W^{\epsilon, p}}+|v|_{\delta, X}^{0, p, \ell}\right)\|w\|_{\delta, X}^{\sigma, \ell},
$$


and for $\sigma_{1}+\sigma_{2}>\ell \delta$, one has

$$
\|R(v, w)\|_{\delta, X}^{\sigma_{1}+\sigma_{2}, \ell} \lesssim\|v\|_{\delta, X}^{\sigma_{1}, \ell}\|w\|_{\delta, X}^{\sigma_{2}, \ell}, \quad|R(v, w)|_{\delta, X}^{\sigma_{1}+\sigma_{2}, p, \ell} \lesssim\|v\|_{\delta, X}^{\sigma_{1}, \ell}|w|_{\delta, X}^{\sigma_{2}, p, \ell}
$$

(V) If $\sigma \in] \ell \delta, 1[$ (or $\sigma \in] \ell \delta-1,1[$ if $\operatorname{div} X=0)$, then one

$$
\|w\|_{\delta, X}^{\sigma, \ell} \sim \sum_{0 \leq i \leq \ell}\left\|\partial_{X}^{i} w\right\|_{C^{\sigma-i \delta}}, \quad \text { and } \quad|w|_{\delta, X}^{\sigma, p, \ell} \sim \mid w \|_{\delta, X}^{\sigma, p, \ell}
$$

The proofs of Lemma 7.1 and Lemma 7.2 will be postponed to the Appendix A. We mention that the main difference between the proof here and that in $[8,9,25]$ is that the Sobolev space $W^{s, p}$ is of Triebel-Lizorkin type space, which is not of Besov type spaces as in the previous papers.

We now present the proof of (3.15).

Proof of (3.15). It is easy to observe from (3.15) and (7.12) that it suffices to prove

$$
\left|\partial_{X_{0}} v_{0}\right|_{\varepsilon / k, X_{0}}^{1-\varepsilon / k, p-1} \leq C\left(\left\|\omega_{0}\right\|_{L^{1} \cap L^{p}}+\left|\omega_{0}\right|_{\varepsilon / k, X_{0}}^{0, p, k}\right) .
$$

As a matter of fact, in view of (3.13) and $p>2$, we have

$$
\left.X_{0} \in C_{\delta}^{1-\delta}\left(X_{0}, k-1\right), \quad \text { for any } \delta \in\right] 0,1[\text { with } \delta k<1
$$

In particular, this gives

$$
\left.X_{0} \in C_{\varepsilon / k}^{1-\varepsilon / k}\left(X_{0}, k-1\right) \quad \text { for any } \varepsilon \in\right] 0,1[.
$$

While for any smooth cut-off function $\zeta(\xi)=\left\{\begin{array}{ll}1, & |\xi| \leq 1, \\ 0, & |\xi| \geq 2,\end{array}\right.$, we deduce from (3.13) and (3.14) that

$$
\left|\partial_{X_{0}} \zeta(D) v_{0}\right|_{\varepsilon / k, X_{0}}^{1-\varepsilon / k, p-1} \leq C\left\|\omega_{0}\right\|_{L^{1} \cap L^{p}} .
$$

So it remains to estimate $\partial_{X_{0}}(I d-\zeta(D)) \nabla^{\perp} \Delta^{-1} \omega_{0}$. Indeed applying Bony's decomposition gives

$$
\begin{aligned}
\partial_{X_{0}}(I d-\zeta(D)) \nabla^{\perp} \Delta^{-1} \omega_{0} & =T_{X_{0} \cdot \nabla}(I d-\zeta(D)) \nabla^{\perp} \Delta^{-1} \omega_{0} \\
& +T_{\nabla(I d-\zeta(D)) \nabla^{\perp} \Delta^{-1} \omega_{0}} \cdot X_{0}+R\left(X_{0}, \nabla(I d-\zeta(D)) \nabla^{\perp} \Delta^{-1} \omega_{0}\right) .
\end{aligned}
$$

Applying (7.7) gives

$$
\left|T_{X_{0} \cdot \nabla}(I d-\zeta(D)) \nabla^{\perp} \Delta^{-1} \omega_{0}\right|_{\varepsilon / k, X_{0}}^{1-\varepsilon / k, p, k-1} \leq\left|(I d-\zeta(D)) \nabla^{\perp} \Delta^{-1} \omega_{0}\right|_{\varepsilon / k, X_{0}}^{1, p, k} \leq C\left|\omega_{0}\right|_{\varepsilon / k, X_{0}}^{0, p, k} .
$$

Applying (7.10) and the property (7.14) with $0<\varepsilon^{\prime}<\varepsilon$ yields

$$
\begin{aligned}
\left|T_{\nabla(I d-\chi(D)) \nabla^{\perp} \Delta^{-1} \omega_{0}} \cdot X_{0}\right|_{\varepsilon / k, X_{0}}^{1-\varepsilon / k, p, k-1} & \leq C\left|\omega_{0}\right|_{\varepsilon / k, X_{0}}^{\left(\varepsilon^{\prime}-\varepsilon\right) / k, p, k-1}\left\|X_{0}\right\|_{\varepsilon / k, X_{0}}^{1-\varepsilon^{\prime} / k, k-1} \\
& \leq C\left|\omega_{0}\right|_{\varepsilon / k, X_{0}}^{0, p, k-1}\left\|X_{0}\right\|_{\varepsilon^{\prime} / k, X_{0}}^{1-\varepsilon^{\prime} / k, k-1} .
\end{aligned}
$$

Finally applying (7.11) and then using (7.7) leads to

$$
\begin{aligned}
\left|R\left(X_{0}, \nabla(I d-\zeta(D)) \nabla^{\perp} \Delta^{-1} \omega_{0}\right)\right|_{\varepsilon / k, X_{0}}^{1-\varepsilon / k, p, k-1} & \\
& \leq C\left|\nabla(I d-\zeta(D)) \nabla^{\perp} \Delta^{-1} \omega_{0}\right|_{\varepsilon / k, X_{0}}^{0, p, k-1}\left\|X_{0}\right\|_{\varepsilon / k, X_{0}}^{1-\varepsilon / k, k-1} \\
& \leq C\left|\omega_{0}\right|_{\varepsilon / k, X_{0}}^{0, p, k}\left\|X_{0}\right\|_{\varepsilon / k, X_{0}}^{1-\varepsilon / k, k-1} .
\end{aligned}
$$

This in turn shows that

$$
\left|\partial_{X_{0}}(I d-\zeta(D)) \nabla^{\perp} \Delta^{-1} \omega_{0}\right|_{\varepsilon / k, X_{0}}^{1-\varepsilon / k, p, k-1} \leq C\left|\omega_{0}\right|_{\varepsilon / k, X_{0}}^{0, p, k}\left\|X_{0}\right\|_{\varepsilon / k, X_{0}}^{1-\varepsilon / k, k-1}
$$


which together with (7.15) gives rise to (7.13).

Appendix A. The proofs of Lemma 7.1 and Lemma 7.2

In this appendix we shall present the proofs of Lemma 7.1 and Lemma 7.2.

Proof of Lemma 7.1. We first prove (7.3) for $\ell=0$. In this case, according to (7.1) and (7.2), for any $i \in\{1, \cdots m\}$, we have

$$
\left|R_{q}\left(\alpha_{1}, \cdots, \alpha_{m}\right)\right| \leq \prod_{j=1, j \neq i}^{m}\left\|\alpha_{j}\right\|_{L^{\infty}} \int_{[0,1]^{m}} \int_{\mathbb{R}^{2}}\left|\alpha_{i}\left(x+f_{i}(\tau) 2^{-q} y\right)\right| H(\tau,|y|) d y d \tau .
$$

Yet similar to the proof of Proposition 1.16 of [5], for any $\tau \in] 0,1[$, one has

$$
\begin{aligned}
& \int_{\mathbb{R}^{2}}\left|\alpha_{i}\left(x+f_{i}(\tau) 2^{-q} y\right)\right| H(\tau,|y|) d y \\
& \quad=\left(\frac{2^{q}}{f_{i}(\tau)}\right)^{2} \int_{0}^{\infty} H\left(\tau, \frac{2^{q} r}{f_{i}(\tau)}\right) \int_{|y|=r}\left|\alpha_{i}(x-y)\right| d \sigma_{r}(y) d r \\
& \quad=-\left(\frac{2^{q}}{f_{i}(\tau)}\right)^{3} \int_{0}^{\infty} \partial_{r} H\left(\tau, \frac{2^{q} r}{f_{i}(\tau)}\right) \int_{B(x, r)}\left|\alpha_{i}(y)\right| d y d r \\
& \quad \leq-\pi\left(\frac{2^{q}}{f_{i}(\tau)}\right)^{3} M\left(\alpha_{i}\right)(x) \int_{0}^{\infty} \partial_{r} H\left(\tau, \frac{2^{q} r}{f_{i}(\tau)}\right) r^{2} d r \\
& =M\left(\alpha_{i}\right)(x)\|H(\tau, \cdot)\|_{L^{1}} .
\end{aligned}
$$

Substituting the above estimate into (A.1) shows that (7.5) holds for $\ell=0$.

Inductively, we assume that (7.3), which implies (7.4), and (7.6), which we shall prove late, hold for $\ell \leq k-1$, we are going to prove that (7.3) holds for $\ell+1$. Note for $\varphi_{1}(\xi)=i \xi \varphi(\xi)$, one has $\sum_{q_{1} \in \mathbb{Z}} 2^{q_{1}} \varphi_{1}\left(2^{-q_{1}} D\right)=\nabla$, which gives (see also the Appendix of [8])

$$
\begin{aligned}
T_{X \cdot \nabla} & R_{q}\left(\alpha_{1}, \cdots, \alpha_{m}\right) \\
= & \sum_{q_{1} \leq q+N_{0}} 2^{q_{1}} S_{q_{1}-1} X \cdot \varphi_{1}\left(2^{-q_{1}} D\right) R_{q}\left(\alpha_{1}, \cdots, \alpha_{m}\right) \\
= & \sum_{q_{1} \leq q+N_{0}} 2^{q_{1}}\left(S_{q_{1}-1} X-S_{q-1} X\right) \cdot \varphi_{1}\left(2^{-q_{1}} D\right) R_{q}\left(\alpha_{1}, \cdots, \alpha_{m}\right) \\
& +\sum_{q_{1} \leq q+N_{0}} 2^{q_{1}}\left(S_{q-1} X-S_{q_{1}-1} X\right) \cdot \sum_{j=1}^{m} R_{q}\left(\alpha_{1}, \cdots, \varphi_{1}\left(2^{-q_{1}} D\right) \alpha_{j}, \cdots, \alpha_{m}\right) \\
& +\sum_{q_{1} \leq q+N_{0}} S_{q_{1}-1} X \cdot \sum_{j=1}^{m} R_{q}\left(\alpha_{1}, \cdots, \nabla \varphi\left(2^{-q_{1}} D\right) \alpha_{j}, \cdots, \alpha_{m}\right) \\
& \stackrel{\text { def }}{=} R_{q}^{1}+R_{q}^{2}+R_{q}^{3} .
\end{aligned}
$$

It follows from the inductive assumption, (7.4), that

$$
\begin{aligned}
&\left|\left(T_{X \cdot \nabla}\right)^{\ell} R_{q}^{1}\right| \lesssim \sum_{\substack{q_{1} \leq q+N_{0} \\
n_{1}, \ell_{1}+\ell_{2} \leq \ell}} 2^{q_{1}} 2^{q \delta\left(\ell-\ell_{1}-\ell_{2}\right)}\left\|\left(T_{X \cdot \nabla}\right)^{\ell_{1}}\left(S_{q_{1}-1} X-S_{q-1} X\right)\right\|_{L^{\infty}} \\
& \times M^{\gamma_{n_{1}}\left(\ell_{2}\right)}\left(\left(T_{X \cdot \nabla}\right)^{\ell_{2}} \varphi_{1}\left(2^{-q_{1}} D\right) R_{q}\left(\alpha_{1}, \cdots, \alpha_{m}\right)\right) .
\end{aligned}
$$


For $q_{1}<q$, applying (7.5) and using assumption that $1-k \delta>0$ yields

$$
\begin{aligned}
\left\|\left(T_{X \cdot \nabla}\right)^{\ell_{1}}\left(S_{q_{1}-1} X-S_{q-1} X\right)\right\|_{L^{\infty}} & \lesssim \sum_{q_{2}=q_{1}-1}^{q-2}\left\|\left(T_{X \cdot \nabla}\right)^{\ell_{1}} \Delta_{q_{2}} X\right\|_{L^{\infty}} \\
& \lesssim \sum_{q_{2}=q_{1}-1}^{q-2} 2^{-q_{2}\left(1-\left(1+\ell_{1}\right) \delta\right)}\|X\|_{\delta, X}^{1-\delta, k-1} \\
& \lesssim 2^{-q_{1}\left(1-\left(1+\ell_{1}\right) \delta\right)}\|X\|_{\delta, X}^{1-\delta, k-1} .
\end{aligned}
$$

Along the same line for $q_{1} \in\left[q, q+N_{0}\right]$, there holds the same estimate for $\|\left(T_{X \cdot \nabla}\right)^{\ell_{1}}\left(S_{q_{1}-1} X-\right.$ $\left.S_{q-1} X\right) \|_{L^{\infty}}$.

Whereas applying (7.6) and then the inductive assumption gives

$$
\begin{aligned}
& \left|\left(T_{X} \cdot \nabla\right)^{\ell_{2}} \varphi_{1}\left(2^{-q_{1}} D\right) R_{q}\left(\alpha_{1}, \cdots, \alpha_{m}\right)\right| \\
& \lesssim \sum_{0 \leq n_{2}, \ell_{3} \leq \ell_{2}} 2^{q_{1} \delta\left(\ell_{2}-\ell_{3}\right)} M^{\gamma_{n_{2}}\left(\ell_{3}\right)}\left(\left(T_{X \cdot \nabla}\right)^{\ell_{3}} R_{q}\left(\alpha_{1}, \cdots, \alpha_{m}\right)\right) \\
& \lesssim \sum_{\substack{|\lambda| \leq \ell_{3} \leq \ell_{2} \\
n_{3} \leq \ell_{3}}} 2^{q\left(\ell_{2}-|\lambda|\right) \delta} \min _{i=1, \cdots, m}\left\{M^{\gamma_{n_{2}}\left(\ell_{3}\right)+\gamma_{n_{3}}\left(\lambda_{i}\right)}\left(\left(T_{X \cdot \nabla}\right)^{\lambda_{i}} \alpha_{i}\right) \prod_{j=1, j \neq i}^{m}\left\|\left(T_{X \cdot \nabla}\right)^{\lambda_{j}} \alpha_{j}\right\|_{L^{\infty}}\right\} .
\end{aligned}
$$

Hence we obtain

$$
\text { (A.2) }\left|\left(T_{X \cdot \nabla}\right)^{\ell} R_{q}^{1}\right| \lesssim \sum_{n,|\lambda| \leq \ell} 2^{q \delta(\ell+1-|\lambda|)} \min _{i=1, \cdots, m}\left\{M^{\gamma_{n}\left(\lambda_{i}\right)}\left(\left(T_{X \cdot \nabla}\right)^{\lambda_{i}} \alpha_{i}\right) \prod_{j \neq i}\left\|\left(T_{X \cdot \nabla}\right)^{\lambda_{j}} \alpha_{j}\right\|_{L^{\infty}}\right\} .
$$

The same argument leads to the same estimate for $R_{q}^{2}$.

Finally noticing that

$$
S_{q_{1}-1} X(x)=S_{q_{1}-1} X\left(x+2^{-q} f_{j}(t) y\right)-\int_{0}^{1} 2^{-q} f_{j}(t) y \cdot \nabla S_{q_{1}-1} X\left(x+\tau 2^{-q} f_{j}(t) y\right) d \tau,
$$

we write

$$
\begin{aligned}
R_{q}^{3}= & \sum_{j=1}^{m} R_{q}\left(\alpha_{1}, \cdots, T_{X \cdot \nabla} \alpha_{j}, \cdots, \alpha_{m}\right) \\
& -\sum_{q_{1} \leq q+N_{0}} 2^{q_{1}-q} \sum_{j=1}^{m} R_{q}^{(j)}\left(\nabla S_{q_{1}-1} X, \alpha_{1}, \cdots, \varphi_{1}\left(2^{-q_{1}} D\right) \alpha_{j}, \cdots, \alpha_{m}\right),
\end{aligned}
$$

where $R_{q}^{(j)}$ is given by

$$
\begin{aligned}
& R_{q}^{(j)}\left(\nabla S_{q_{1}-1} X, \alpha_{1}, \cdots, \varphi_{1}\left(2^{-q_{1}} D\right) \alpha_{j}, \cdots, \alpha_{m}\right) \\
& =\int_{0}^{1} \int_{[0,1]^{m}} \int_{\mathbb{R}^{d}} \nabla S_{q-1} X\left(x+\tau 2^{-q} f_{j}(t) y\right) \\
& \quad \times \varphi_{1}\left(2^{-q} D\right) \alpha_{j}\left(x+2^{-q} f_{j}(t) y\right) \prod_{i \neq j} \alpha_{i}\left(x+2^{-q} f_{i}(t) y\right) \cdot y f_{j}(t) h(t, y) d y d t d \tau .
\end{aligned}
$$

Using the inductive assumption and taking into account the fact that

$$
\left\|\left(T_{X \cdot \nabla}\right)^{\mu} \nabla S_{q_{1}-1} X\right\|_{L^{\infty}} \lesssim \sum_{q_{2} \leq q_{1}-2} 2^{q_{2} \delta(1+\mu)}\|\nabla X\|_{\delta, X}^{-\delta, \mu} \lesssim 2^{q_{1} \delta(1+\mu)}\|X\|_{\delta, X}^{1-\delta, k-1}, \quad \mu \leq \ell \leq k-1,
$$


we write

$$
\begin{aligned}
& \left|\left(T_{X \cdot \nabla}\right)^{\ell} R_{q}^{(j)}\left(\nabla S_{q_{1}-1} X, \alpha_{1}, \cdots, \varphi_{1}\left(2^{-q_{1}} D\right) \alpha_{j}, \cdots, \alpha_{m}\right)\right| \\
& \lesssim \sum_{n, \mu+|\lambda| \leq \ell} 2^{q(\ell-\mu-|\lambda|) \delta}\left\|\left(T_{X \cdot \nabla}\right)^{\mu} \nabla S_{q_{1}-1} X\right\|_{L^{\infty}} \\
& \times \min _{i=1, \cdots, m}\left\{M^{\gamma_{n}\left(\lambda_{i}\right)}\left(\left(T_{X \cdot \nabla}\right)^{\lambda_{i}} \bar{\alpha}_{i}\right) \prod_{j=1, j \neq i}^{m}\left\|\left(T_{X \cdot \nabla}\right)^{\lambda_{j}} \bar{\alpha}_{j}\right\|_{L^{\infty}}\right\} \\
& \lesssim \sum_{n,|\lambda| \leq \ell} 2^{q(\ell+1-|\lambda|) \delta}\|X\|_{\delta, X}^{1-\delta, k-1} \min _{i=1, \cdots, m}\left\{M^{\gamma_{n}\left(\lambda_{i}\right)}\left(\left(T_{X \cdot \nabla}\right)^{\lambda_{i}} \alpha_{i}\right) \prod_{j=1, j \neq i}^{m}\left\|\left(T_{X \cdot \nabla}\right)^{\lambda_{j}} \alpha_{j}\right\|_{L^{\infty}}\right\}
\end{aligned}
$$

where $\bar{\alpha}_{i}=\left\{\begin{array}{ll}\alpha_{i}, & i \neq j, \\ \varphi_{1}\left(2^{-q_{1}} D\right) \alpha_{j}, & i=j,\end{array}\right.$ and we used (7.5) and (7.6) in the last step. This together with (A.3) and the inductive assumption ensures that that $R_{q}^{3}$ shares the same estimate as $R_{q}^{1}$ in (A.2). This completes the proof of Lemma 7.1.

Next let us turn to the proof of Lemma 7.2.

Proof of Lemma 7.2. The statements concerning the norm $\|\cdot\|_{\delta, X}^{\sigma, \ell}$ have been proved in Section 2.2 of [8], Theorem 3.2.2 of [9] and Lemma 2.2 of [25]. We just need to prove the estimates for the norm $|\cdot|_{\delta, X}^{\sigma, p, \ell}$. Firstly the Estimate (7.4) follows directly from (7.3).

- Proof of (7.6)

In view of Proposition 1.16 and Remark 1.17 of [5], (7.6) holds for $\ell=0$. Inductively, let us assume that (7.6) holds for $\ell \leq k-1$, we are going to prove this estimate for $\ell+1$. Notice that

$$
\left(T_{X \cdot \nabla}\right)^{\ell+1} \theta\left(2^{-q} D\right)(w)=\left(T_{X \cdot \nabla}\right)^{\ell}\left[T_{X \cdot \nabla} ; \theta\left(2^{-q} D\right)\right] w+\left(T_{X \cdot \nabla}\right)^{\ell} \theta\left(2^{-q} D\right)\left(T_{X \cdot \nabla} w\right) .
$$

It is easy to observe from the spectral properties to terms of Bony's decomposition that there exist a smooth function $\tilde{\theta}$ with compact support in some annulus of $\mathbb{R}^{2}$ and a fixed integer $N_{\theta}$ so that

$$
\begin{aligned}
{\left[T_{X \cdot \nabla} ; \theta\left(2^{-q} D\right)\right] w } & =\sum_{\left|q_{1}-q\right| \leq N_{\theta}}\left[S_{q_{1}-1} X ; \theta\left(2^{-q} D\right)\right] \varphi\left(2^{-q_{1}} D\right) \nabla \tilde{\theta}\left(2^{-q} D\right) w \\
= & 2^{q} \sum_{\left|q_{1}-q\right| \leq N_{\theta}}\left(S_{q_{1}-1} X-S_{q-1} X\right) \cdot \theta\left(2^{-q} D\right) \varphi\left(2^{-q_{1}} D\right) \tilde{\theta}_{1}\left(2^{-q} D\right) w \\
& +2^{q}\left[S_{q-1} X ; \theta\left(2^{-q} D\right)\right] \tilde{\theta}_{1}\left(2^{-q} D\right) w \\
& -2^{q} \sum_{\left|q_{1}-q\right| \leq N_{\theta}} \theta\left(2^{-q} D\right)\left(\left(S_{q_{1}-1} X-S_{q-1} X\right) \cdot \varphi\left(2^{-q_{1}} D\right) \tilde{\theta}_{1}\left(2^{-q} D\right) w\right) \\
& \stackrel{\operatorname{def}}{=} T_{q}^{1}+T_{q}^{2}+T_{q}^{3},
\end{aligned}
$$

where $\tilde{\theta}_{1}(\xi) \stackrel{\text { def }}{=} i \xi \tilde{\theta}(\xi)$. 
It follows form (7.4) and (7.5) that

$$
\begin{aligned}
\left|\left(T_{X \cdot \nabla}\right)^{\ell} T_{q}^{1}\right| \lesssim \sum_{n_{1}, \lambda_{1}+\lambda_{2} \leq \ell} 2^{q \delta\left(\ell-\lambda_{1}-\lambda_{2}\right)} 2^{q} \sum_{\left|q_{1}-q\right| \leq N_{\theta}}\left\|\left(T_{X \cdot \nabla}\right)^{\lambda_{1}}\left(S_{q_{1}-1} X-S_{q-1} X\right)\right\|_{L^{\infty}} \\
\times M^{\gamma_{n_{1}}\left(\lambda_{2}\right)}\left(\left(T_{X \cdot \nabla}\right)^{\lambda_{2}} \theta\left(2^{-q} D\right) \varphi\left(2^{-q_{1}} D\right) \tilde{\theta}_{1}\left(2^{-q} D\right) w\right) \\
\lesssim \sum_{n_{1}, \lambda_{1}+\lambda_{2} \leq \ell} 2^{q \delta\left(\ell-\lambda_{1}-\lambda_{2}\right)} 2^{q} 2^{q\left(\lambda_{1} \delta-(1-\delta)\right)}\|X\|_{\delta, X}^{1-\delta, k-1} \\
\times M^{\gamma_{n_{1}}\left(\lambda_{2}\right)}\left(\left(T_{X \cdot \nabla}\right)^{\lambda_{2}} \theta\left(2^{-q} D\right) \varphi\left(2^{-q_{1}} D\right) \tilde{\theta}_{1}\left(2^{-q} D\right) w\right),
\end{aligned}
$$

which together with the inductive assumption implies

$$
\left|\left(T_{X \cdot \nabla}\right)^{\ell} T_{q}^{1}\right| \lesssim \sum_{n, \lambda \leq \ell} 2^{q \delta(\ell+1-\lambda)} M^{\gamma_{n}(\lambda)}\left(\left(T_{X \cdot \nabla}\right)^{\lambda} w\right)
$$

Furthermore, there holds

$$
\begin{aligned}
& \left\|\left(2^{q(s-(\ell+1) \delta)}\left(T_{X \cdot \nabla}\right)^{\ell} T_{q}^{1}\right)_{\ell^{2}(\mathbb{N})}\right\|_{L^{p}} \\
& \quad \lesssim \sum_{\lambda \leq \ell}\left\|\left(2^{q(s-\lambda \delta)} M^{\gamma(\lambda)}\left(\left(T_{X} \cdot \nabla\right)^{\lambda} \theta\left(2^{-q} D\right) w\right)\right)_{\ell^{2}(\mathbb{N})}\right\|_{L^{p}} \\
& \quad \lesssim \sum_{\lambda \leq \ell}\left\|\left(2^{q(s-\lambda \delta)}\left(T_{X \cdot \nabla}\right)^{\lambda} \theta\left(2^{-q} D\right) w\right)_{\ell^{2}(\mathbb{N})}\right\|_{L^{p}} \lesssim|w|_{\delta, X}^{s, p, \ell} .
\end{aligned}
$$

Whereas by applying (7.6) for $\lambda \leq \ell$ and (7.4), one has

$$
\begin{aligned}
\left|\left(T_{X \cdot \nabla}\right)^{\ell} T_{q}^{3}\right| \lesssim 2^{q} \sum_{\substack{\left|q_{1}-q\right| \leq N_{\theta} \\
n_{1}, \lambda \leq \ell}} 2^{q \delta(\ell-\lambda)} M^{\gamma_{n_{1}}(\lambda)}\left(\left(T_{X \cdot \nabla}\right)^{\lambda}\left(\left(S_{q_{1}-1} X-S_{q-1} X\right) \varphi\left(2^{-q_{1}} D\right) \tilde{\theta}_{1}\left(2^{-q} D\right) w\right)\right) \\
\lesssim \sum_{\substack{\left|q_{1}-q\right| \leq N_{\theta}, n_{1} \leq \ell \\
n_{2}, \lambda_{1}+\lambda_{2} \leq \lambda \leq \ell}} 2^{q \delta\left(\ell-\lambda_{1}-\lambda_{2}\right)} 2^{q}\left\|\left(T_{X \cdot \nabla}\right)^{\lambda_{1}}\left(S_{q_{1}-1} X-S_{q-1} X\right)\right\|_{L^{\infty}} \\
\times M^{\gamma_{n_{1}}(\lambda)+\gamma_{n_{2}}\left(\lambda_{2}\right)}\left(\left(T_{X \cdot \nabla}\right)^{\lambda_{2}} \varphi\left(2^{-q_{1}} D\right) \tilde{\theta}_{1}\left(2^{-q} D\right) w\right) .
\end{aligned}
$$

Then the Estimates (A.4)-(A.5) also hold for $T_{q}^{3}$.

To deal with $T_{q}^{2}$, let us denote $h_{\theta} \stackrel{\text { def }}{=} \mathcal{F}^{-1} \theta$, then one has

$$
\begin{aligned}
T_{q}^{2} & =2^{3 q} \int_{\mathbb{R}^{2}} h_{\theta}\left(2^{q}(x-y)\right)\left(S_{q-1} X(x)-S_{q-1} X(y)\right) \cdot \tilde{\theta}_{1}\left(2^{-q} D\right) w(y) d y \\
& =\int_{\mathbb{R}^{2}} \int_{0}^{1} h_{\theta}(z) z \cdot \nabla S_{q-1} X\left(x+(1-t) 2^{-q} z\right) \cdot \tilde{\theta}_{1}\left(2^{-q} D\right) w\left(x-2^{-q} z\right) d t d z,
\end{aligned}
$$

so that by applying (7.3), we obtain

$$
\left|\left(T_{X \cdot \nabla}\right)^{\ell} T_{q}^{2}\right| \lesssim \sum_{n, \lambda_{1}+\lambda_{2} \leq \ell} 2^{q \delta\left(\ell-\lambda_{1}-\lambda_{2}\right)}\left\|\left(T_{X \cdot \nabla}\right)^{\lambda_{1}}\left(\nabla S_{q-1} X\right)\right\|_{L^{\infty}} M^{\gamma_{n}\left(\lambda_{2}\right)}\left(\left(T_{X \cdot \nabla}\right)^{\lambda_{2}} \tilde{\theta}_{1}\left(2^{-q} D\right) w\right) .
$$

Yet applying (7.5) yields

$$
\left\|\left(T_{X \cdot \nabla}\right)^{\lambda_{1}}\left(\nabla S_{q-1} X\right)\right\|_{L^{\infty}} \lesssim 2^{q \delta\left(1+\lambda_{1}\right)}\|X\|_{\delta, X}^{1-\delta, k-1},
$$

from which and the inductive assumption, we conclude that the Estimates (A.4)-(A.5) hold for $T_{q}^{2}$ as well. 
Finally note that

$$
\left|T_{X \cdot \nabla} w\right|_{\delta, X}^{s-\delta, p, \ell} \lesssim|w|_{\delta, X}^{s, p, \ell+1}
$$

we deduce from the inductive assumption that

$$
\begin{aligned}
& \left|\left(T_{X \cdot \nabla}\right)^{\ell} \theta\left(2^{-q} D\right)\left(T_{X \cdot \nabla} w\right)\right| \lesssim \sum_{n, \lambda \leq \ell} 2^{q((\ell+1)-(\lambda+1))} M^{\gamma_{n}(\lambda+1)}\left(\left(T_{X \cdot \nabla}\right)^{\lambda+1} w\right) \quad \text { and } \\
& \left\|\left(2^{q((s-\delta)-\ell \delta)}\left(T_{X \cdot \nabla}\right)^{\ell} \theta\left(2^{-q} D\right)\left(T_{X \cdot \nabla} w\right)\right)_{\ell^{2}(\mathbb{N})}\right\|_{L^{p}} \lesssim|w|_{\delta, X}^{s, p, \ell+1} .
\end{aligned}
$$

This proves (7.6) holds for $\ell+1$.

- Proof of (7.7) Without loss of generality, we may assume that $\operatorname{supp} \chi \subset\{x:|\xi| \geq M\}$. Then it is easy to observe that there exists a smooth function $\varphi_{\chi}$ with compact support in some annulus of $\mathbb{R}^{2}$ such that for $q \geq 0$

$$
\Delta_{q}\left(T_{X \cdot \nabla}\right)^{\ell} \chi(D) w=2^{q m} \Delta_{q}\left(T_{X \cdot \nabla}\right)^{\ell} \varphi_{\chi}\left(2^{-q} D\right) w .
$$

Then we infer

$$
\begin{aligned}
& \left\|\left(T_{X \cdot \nabla}\right)^{\ell} \chi(D) w\right\|_{W^{s-m-\ell \delta, p}}=\left\|\left(2^{q(s-m-\ell \delta)} \Delta_{q}\left(T_{X \cdot \nabla}\right)^{\ell} \chi(D) w\right)_{\ell^{2}(\mathbb{N})}\right\|_{L^{p}} \\
& =\left\|\left(2^{q(s-\ell \delta)} \Delta_{q}\left(T_{X \cdot \nabla}\right)^{\ell} \varphi_{\chi}\left(2^{-q} D\right) w\right)_{\ell^{2}(\mathbb{N})}\right\|_{L^{p}} \\
& \lesssim\left\|\left(2^{q(s-\ell \delta)} M\left(\left(T_{X \cdot \nabla}\right)^{\ell} \varphi_{\chi}\left(2^{-q} D\right) w\right)\right)_{\ell^{2}(\mathbb{N})}\right\|_{L^{p}} \\
& \lesssim\left\|\left(2^{q(s-\ell \delta)}\left(T_{X \cdot \nabla}\right)^{\ell} \varphi_{\chi}\left(2^{-q} D\right) w\right)_{\ell^{2}(\mathbb{N})}\right\|_{L^{p}} \\
& \lesssim|w|_{\delta, X}^{s, p, \ell} \text {, by }(7.6) \text {. }
\end{aligned}
$$

And (7.7) follows.

- Proof of the para-product estimates. Due to the support properties to the Fourier transform of the terms in the para-product decomposition, for any integer $\ell$, there exists a positive integer $N_{\ell}$ so that

$$
\Delta_{q}\left(T_{X \cdot \nabla}\right)^{\ell} T_{v} w=\Delta_{q} \sum_{\left|q_{1}-q\right| \leq N_{\ell}}\left(T_{X \cdot \nabla}\right)^{\ell}\left(S_{q_{1}-1} v \Delta_{q_{1}} w\right)
$$

Applying (7.4) gives

$$
\begin{aligned}
\left|\Delta_{q}\left(T_{X \cdot \nabla}\right)^{\ell} T_{v} w\right| & \lesssim \sum_{\substack{\left|q_{1}-q\right| \leq N\\
}} M\left(\left(T_{X \cdot \nabla}\right)^{\ell}\left(S_{q_{1}-1} v \Delta_{q_{1}} w\right)\right) \\
& \lesssim \sum_{\substack{\left|q_{1}-q\right| \leq N \\
n, \lambda_{1}+\lambda_{2} \leq \ell}} 2^{q_{1} \delta\left(\ell-\lambda_{1}-\lambda_{2}\right)}\left\|\left(T_{X \cdot \nabla}\right)^{\lambda_{1}} S_{q_{1}-1} v\right\|_{L^{\infty}} M^{\gamma_{n}\left(\lambda_{2}\right)+1}\left(\left(T_{X \cdot \nabla}\right)^{\lambda_{2}} \Delta_{q_{1}} w\right) .
\end{aligned}
$$

Yet it follows from (7.5) that

$$
\left\|\left(T_{X \cdot \nabla}\right)^{\lambda_{1}} S_{q_{1}-1} v\right\|_{L^{\infty}} \lesssim\left\{\begin{array}{l}
2^{q_{1}\left(-\sigma+\lambda_{1} \delta\right)}\|v\|_{\delta, X}^{\sigma, \ell} \text { if } \sigma<0 \\
\|v\|_{L^{\infty}} \text { if } \lambda_{1}=0 \\
2^{q_{1} \lambda_{1} \delta}\|v\|_{\delta, X}^{0, k} \text { if } \lambda_{1}>0
\end{array}\right.
$$


Therefore, we get, by applying (7.6) that for $\sigma<0$

$$
\begin{aligned}
\left\|\left(T_{X \cdot \nabla}\right)^{\ell} T_{v} w\right\|_{W^{s+\sigma-\ell \delta, p}} & =\left\|\left(2^{q(s+\sigma-\ell \delta)} \Delta_{q}\left(T_{X \cdot \nabla}\right)^{\ell} T_{v} w\right)_{\ell^{2}(\mathbb{N})}\right\|_{L^{p}} \\
& \lesssim\|v\|_{\delta, X}^{\sigma, \ell} \sum_{\lambda_{2} \leq \ell}\left\|\left(2^{q\left(s-\lambda_{2} \delta\right)}\left(T_{X \cdot \nabla}\right)^{\lambda_{2}} \Delta_{q_{1}} w\right)_{\ell^{2}(\mathbb{N})}\right\|_{L^{p}} \\
& \lesssim\|v\|_{\delta, X}^{\sigma, \ell}|w|_{\delta, X}^{s, p, \ell}
\end{aligned}
$$

and

$$
\begin{aligned}
\left\|\left(T_{X \cdot \nabla}\right)^{\ell} T_{v} w\right\|_{W^{s-\ell \delta, p}} & =\left\|\left(2^{q(s-\ell \delta)} \Delta_{q}\left(T_{X \cdot \nabla}\right)^{\ell} T_{v} w\right)_{\ell^{2}(\mathbb{N})}\right\|_{L^{p}} \\
& \lesssim\left(\|v\|_{L^{\infty}}+\|v\|_{\delta, X}^{0, \ell}\right) \sum_{\lambda_{2} \leq \ell}\left\|\left(2^{q\left(s-\lambda_{2} \delta\right)}\left(T_{X \cdot \nabla}\right)^{\lambda_{2}} \Delta_{q_{1}} w\right)_{\ell^{2}(\mathbb{N})}\right\|_{L^{p}} \\
& \lesssim\left(\|v\|_{L^{\infty}}+\|v\|_{\delta, X}^{0, \ell}\right)|w|_{\delta, X}^{s, p, \ell}
\end{aligned}
$$

This proves (7.9).

By the same manner, we have for $s<0$

$$
\begin{aligned}
\left\|\left(T_{X \cdot \nabla}\right)^{\ell} T_{v} w\right\|_{W^{\sigma+s-\ell \delta, p}} & =\left\|\left(2^{q(\sigma+s-\ell \delta)} \Delta_{q}\left(T_{X \cdot \nabla}\right)^{\ell} T_{v} w\right)_{\ell^{2}(\mathbb{N})}\right\|_{L^{p}} \\
& \lesssim\|w\|_{\delta, X}^{\sigma, \ell} \sum_{\lambda_{1} \leq \ell}\left\|\left(\sum_{q_{2} \leq q+N} 2^{\left(q-q_{2}\right)\left(s-\lambda_{1} \delta\right)} 2^{q_{2}\left(s-\lambda_{1} \delta\right)}\left(T_{X \cdot \nabla}\right)^{\lambda_{1}} \Delta_{q_{2}} v\right)_{\ell^{2}(\mathbb{N})}\right\|_{L^{p}} \\
& \lesssim\|w\|_{\delta, X}^{\sigma, \ell} \sum_{\lambda_{1} \leq \ell}\left\|\left(2^{q\left(s-\lambda_{1} \delta\right)}\left(T_{X \cdot \nabla}\right)^{\lambda_{1}} \Delta_{q} v\right)_{\ell^{2}(\mathbb{N})}\right\|_{L^{p}} \\
& \lesssim|v|_{\delta, X}^{s, p, \ell}\|w\|_{\delta, X}^{\sigma, \ell} .
\end{aligned}
$$

Notice that when for $s=0$, one has

$$
\left\|\left(\sum_{q_{2} \leq q+N} 2^{-\left(q-q_{2}\right) \lambda_{1} \delta} 2^{-q_{2} \lambda_{1} \delta}\left(T_{X \cdot \nabla}\right)^{\lambda_{1}} \Delta_{q_{2}} v\right)_{\ell^{2}(\mathbb{N})}\right\|_{L^{p}} \lesssim \begin{cases}\|v\|_{\delta, X}^{0, \ell} & \text { if } \lambda_{1}>0 \\ C_{\epsilon}\|v\|_{W^{\epsilon, p}} & \text { if } \lambda_{1}=0 .\end{cases}
$$

This yields the second estimate of (7.10).

To handle the estimate of (7.11), we write

$$
\Delta_{q}\left(T_{X \cdot \nabla}\right)^{\ell} R(v, w)=\Delta_{q} \sum_{q_{1} \geq q-N}\left(T_{X \cdot \nabla}\right)^{\ell} \Delta_{q_{1}} v \tilde{\Delta}_{q_{1}} w
$$

from which and (7.5), we infer

$$
\begin{aligned}
\left|\Delta_{q}\left(T_{X \cdot \nabla}\right)^{\ell} R(v, w)\right| & \lesssim \sum_{q_{1} \geq q-N} M\left(\left(T_{X \cdot \nabla}\right)^{\ell}\left(\Delta_{q_{1}} v \tilde{\Delta}_{q_{1}} w\right)\right) \\
& \lesssim \sum_{\substack{q_{1} \geq q-N \\
n, \lambda_{1}+\lambda_{2} \leq \ell}} 2^{q_{1} \delta\left(\ell-\lambda_{1}-\lambda_{2}\right)}\left\|\left(T_{X \cdot \nabla}\right)^{\lambda_{1}} \Delta_{q_{1}} v\right\|_{L^{\infty}} M^{\gamma_{n}\left(\lambda_{2}\right)+1}\left(\left(T_{X} \cdot \nabla\right)^{\lambda_{2}} \tilde{\Delta}_{q_{1}} w\right) \\
& \lesssim \sum_{\substack{q_{1} \geq q-N \\
n, \lambda_{2} \leq \ell}} 2^{-q_{1}\left(\sigma_{1}-\left(\ell-\lambda_{2}\right) \delta\right)}\|v\|_{\delta, X}^{\sigma_{1}, \ell} M^{\gamma_{n}\left(\lambda_{2}\right)+1}\left(\left(T_{X \cdot \nabla}\right)^{\lambda_{2}} \tilde{\Delta}_{q_{1}} w\right) .
\end{aligned}
$$


Then we get, by applying (7.6), that

$$
\begin{aligned}
& \left\|\left(T_{X \cdot \nabla}\right)^{\ell} R(v, w)\right\|_{W^{\sigma_{1}+\sigma_{2}-\ell \delta, p}} \\
& =\left\|\left(2^{q\left(\sigma_{1}+\sigma_{2}-\ell \delta\right)} \Delta_{q}\left(T_{X \cdot \nabla}\right)^{\ell} R(v, w)\right)_{\ell^{2}(\mathbb{N})}\right\|_{L^{p}} \\
& \lesssim\|v\|_{\delta, X}^{\sigma_{1}, \ell} \sum_{n, \lambda_{2} \leq \ell} \|\left(\sum_{q_{1} \geq q-N} 2^{\left(q-q_{1}\right)\left(\sigma_{1}+\sigma_{2}-\ell \delta\right)} 2^{q_{1}\left(\sigma_{2}-\lambda_{2} \delta\right)} M^{\gamma_{n}\left(\lambda_{2}\right)+1}\left(\left(T_{X \cdot \nabla}\right)^{\lambda_{2}} \tilde{\Delta}_{q_{1}} w\right)_{\ell^{2}(\mathbb{N})} \|_{L^{p}}\right. \\
& \lesssim\|v\|_{\delta, X}^{\sigma_{1}, \ell} \sum_{n, \lambda_{2} \leq \ell} \|\left(2^{q\left(\sigma_{2}-\lambda_{2} \delta\right)} M^{\gamma_{n}\left(\lambda_{2}\right)+1}\left(\left(T_{X \cdot \nabla}\right)^{\lambda_{2}} \tilde{\Delta}_{q_{1}} w\right)_{\ell^{2}(\mathbb{N})} \|_{L^{p}} \text { if } \sigma_{1}+\sigma_{2}>\ell \delta\right. \\
& \lesssim\|v\|_{\delta, X}^{\sigma_{1}, l}|w|_{\delta, X}^{\sigma_{2}, p, \ell} .
\end{aligned}
$$

This proves (7.11).

-Proof of (7.12) It is easy to observe that (7.12) holds for $\ell=0$. Inductively we assume that (7.12) holds for $\ell$ provided that $\sigma \in] \ell \delta, 1[$ or $\sigma \in] \ell \delta-1,1[$ if $\operatorname{div} X=0$. We next show that

$$
\left\|\left(T_{X \cdot \nabla}\right)^{\ell+1} w\right\|_{W^{\sigma-(\ell+1) \delta, p}} \lesssim \mid w \|_{\delta, X}^{\sigma, p, \ell+1} \text { and }\left\|\partial_{X}^{\ell+1} w\right\|_{W^{\sigma-(\ell+1) \delta, p}} \lesssim|w|_{\delta, X}^{\sigma, p, \ell+1}
$$

provided that

$$
\sigma \in](\ell+1) \delta, 1[\text { or } \sigma \in](\ell+1) \delta-1,1[\text { if } \operatorname{div} X=0 .
$$

It is easy to observe that

$$
\left(T_{X \cdot \nabla}\right)^{\ell+1} w=\left(T_{X \cdot \nabla}\right)^{\ell}\left(T_{X \cdot \nabla}-\partial_{X}\right) w+\left(T_{X \cdot \nabla}\right)^{\ell} \partial_{X} w
$$

And by using Bony's decomposition, one has

$$
\begin{aligned}
\left(\partial_{X}-T_{X \cdot \nabla}\right) w & =T_{\nabla w} \cdot X+R(X, \nabla w) \\
& =T_{\nabla w} \cdot X+\operatorname{div} R(X, w)-R(\operatorname{div} X, w) .
\end{aligned}
$$

Due to $\sigma<1$, applying (7.10) yields

$$
\begin{aligned}
\left\|\left(T_{X \cdot \nabla}\right)^{\ell} T_{\nabla w} \cdot X\right\|_{W^{\sigma-(\ell+1) \delta, p}} & \lesssim\left|T_{\nabla w} \cdot X\right|_{\delta, X}^{\sigma-\delta, p, \ell} \\
& \lesssim|\nabla w|_{\delta, X}^{\sigma-1, p, \ell}\|X\|_{\delta, X}^{1-\delta, \ell} \lesssim|w|_{\delta, X}^{\sigma, p, \ell}\|X\|_{\delta, X}^{1-\delta, \ell} .
\end{aligned}
$$

Similarly whenever, $\sigma>-1-(\ell+1) \delta$, applying (7.7) and (7.11) leads to

$$
\begin{aligned}
\left\|\left(T_{X \cdot \nabla}\right)^{\ell} \operatorname{div} R(X, w)\right\|_{W^{\sigma-(\ell+1) \delta, p}} & \lesssim|\operatorname{div} R(X, w)|_{\delta, X}^{\sigma-\delta, p, \ell} \\
& \lesssim|R(X, w)|_{\delta, X}^{1+\sigma-\delta, p, \ell} \lesssim|w|_{\delta, X}^{\sigma, p, \ell}\|X\|_{\delta, X}^{1-\delta, \ell}
\end{aligned}
$$

The same estimate holds for $\left(T_{X \cdot \nabla}\right)^{\ell} R(\operatorname{div} X, w)$ provided that $\sigma>(\ell+1) \delta$. So that under the assumption (A.7), we have

$$
\left\|\left(T_{X \cdot \nabla}\right)^{\ell}\left(T_{X \cdot \nabla}-\partial_{X}\right) w\right\|_{W^{\sigma-(\ell+1) \delta, p}} \lesssim|w|_{\delta, X}^{\sigma, p, \ell}\|X\|_{\delta, X}^{1-\delta, \ell} .
$$

While it follows from inductive assumption, $|w|_{\delta, X}^{\sigma, p, \ell} \lesssim|w|_{\delta, X}^{\sigma, p, \ell}$, that

$$
\left\|\left(T_{X \cdot \nabla}\right)^{\ell} \partial_{X} w\right\|_{W^{\sigma-(\ell+1) \delta, p}} \lesssim\left|\partial_{X} w\right|_{\delta, X}^{\sigma-\delta, p, \ell} \lesssim\left|\partial_{X} w\left\|_{\delta, X}^{\sigma-\delta, p, \ell} \lesssim \mid w\right\|_{\delta, X}^{\sigma, p, \ell+1} .\right.
$$

This together with (A.8) proves the first estimate of (A.6). 
By the same manner, using the inductive assumptions and (A.8), we deduce that under the condition (A.7),

$$
\begin{aligned}
\left\|\partial_{X}^{\ell+1} w\right\|_{W^{\sigma-(\ell+1) \delta, p}} & \lesssim\left\|\partial_{X}^{\ell}\left(\partial_{X} w-T_{X \cdot \nabla} w\right)\right\|_{W^{\sigma-(\ell+1) \delta, p}}+\left\|\partial_{X}^{\ell} T_{X \cdot \nabla} w\right\|_{W^{\sigma-(\ell+1) \delta, p}} \\
& \lesssim\left|\partial_{X} w-T_{X \cdot \nabla} w\right|_{\delta, X}^{\sigma-\delta, p, \ell}+\left|T_{X \cdot \nabla} w\right|_{\delta, X}^{\sigma-\delta, p, \ell} \\
& \lesssim|w|_{\delta, X}^{\sigma, p, \ell}\|X\|_{\delta, X}^{1-\delta, \ell}+|w|_{\delta, X}^{\sigma, p, \ell+1} \lesssim|w|_{\delta, X}^{\sigma, p, \ell+1} .
\end{aligned}
$$

This proves the second estimate of (A.6). Thus (7.12) follows. This completes the proof of Lemma 7.2.

Acknowledgments. We would like to thank Professor Jean-Yves Chemin for careful reading and profitable suggestions on the preliminary version of this paper. Part of this work was done when we were visiting Morningside Center of the Academy of Mathematics and Systems Sciences, CAS. We appreciate the hospitality and the financial support from MCM. P. Zhang is partially supported by NSF of China under Grant 11371347, the fellowship from Chinese Academy of Sciences and innovation grant from National Center for Mathematics and Interdisciplinary Sciences.

\section{REFERENCES}

[1] H. Abidi, Équations de Navier-Stokes avec densité et viscosité variables dans l'espace critique, Rev. Mat. Iberoam., 23 (2007), 537-586.

[2] H. Abidi and M. Paicu, Existence globale pour un fluide inhomogène, Ann. Inst. Fourier, 57 (2007), 883-917.

[3] H. Abidi, G. Gui and P. Zhang, On the wellposedness of 3-D inhomogeneous Navier-Stokes equations in the critical spaces, Arch. Ration. Mech. Anal., 204 (2012), 189-230.

[4] H. Abidi, G. Gui and P. Zhang, Wellposedness of 3-D inhomogeneous Navier-Stokes equations with highly oscillating initial velocity field, J. Math. Pures Appl., 100 (2013), 166-203 .

[5] H. Bahouri, J.-Y. Chemin and R. Danchin, Fourier Analysis and Nonlinear Partial Differential Equations, Grundlehren der Mathematischen Wissenschaften, Springer, 2011.

[6] A.-L. Bertozzi and P. Constantin, Global regularity for vortex patches, Comm. Math. Phys., 152 (1993), $19-28$.

[7] J.-M. Bony, Calcul symbolique et propagation des singularités pour les équations aux dérivées partielles non linéaires, Ann. Sci. École Norm. Sup., 14 (1981), 209-246.

[8] J.-Y. Chemin, Calcul paradifférentiel précisé et applications à des équations aux dérivées partielles non semilinéaires, Duke Math. J., 56 (1988), 431-469.

[9] J.-Y. Chemin, Sur le mouvement des particules d'un fluide parfait incompressible bidimensionnel, Invent. Math., 103 (1991), 599-629.

[10] J.-Y. Chemin, Persistance de structures géomtriques dans les fluides incompressibles bidimensionnels, Ann. Sci. École Norm. Sup., 26 (1993), 517-542.

[11] J.-Y. Chemin, Perfect incompressible fluids, Oxford Lecture Series in Mathematics and its Applications, 14. The Clarendon Press, Oxford University Press, New York, 1998.

[12] R. Danchin, Poches de tourbillon visqueuses, J. Math. Pures Appl. (9), 76 (1997), 609-647.

[13] R. Danchin, Density-dependent incompressible viscous fluids in critical spaces, Proc. Roy. Soc. Edinburgh Sect. A, 133 (2003), 1311-1334.

[14] R. Danchin and P. Mucha, A Lagrangian approach for the incompressible Navier-Stokes equations with variable density, Comm. Pure Appl. Math., 65 (2012), 1458-1480.

[15] R. Danchin and P. Zhang, Inhomogeneous Navier-Stokes equations in the half-space, with only bounded density, J. Funct. Anal., 267 (2014), 2371-2436.

[16] P. Gamblin and X. Saint-Raymond, On three-dimensional vortex patches, Bull. Soc. Math. France, 123 (1995), 375-424.

[17] T. Hmidi, Régularité Höldérienne des poches de tourbillon visqueuses, J. Math. Pures Appl. (9), 84 (2005), 1455-1495. 
[18] T. Hmidi, Poches de tourbillon singuliéres dans un fluide faiblement visqueux, Rev. Mat. Iberoam., 22 (2006), 489-543.

[19] J. Huang, M. Paicu and P. Zhang, Global well-posedness of incompressible inhomogeneous fluid systems with bounded density or non-Lipschitz velocity, Arch. Ration. Mech. Anal., 209 (2013), 631-682.

[20] O.-A. Ladyvzenskaja and V.-A. Solonnikov, The unique solvability of an initial-boundary value problem for viscous incompressible inhomogeneous fluids. (Russian) Boundary value problems of mathematical physics, and related questions of the theory of functions, 8, Zap. Nauvcn. Sem. Leningrad. Otdel. Mat. Inst. Steklov. (LOMI), 52 (1975), 52-109, 218-219.

[21] P. G. Lemarié-Rieusset, Recent developments in the Navier-Stokes problem, Chapman \& Hall/CRC Research Notes in Mathematics, 431, 2002 .

[22] P. L. Lions, Mathematical topics in fluid mechanics. Vol. 1. Incompressible models. Oxford Lecture Series in Mathematics and its Applications, 3. Oxford Science Publications. The Clarendon Press, Oxford University Press, New York, 1996

[23] M. Paicu and P. Zhang, Global solutions to the 3-D incompressible inhomogeneous Navier-Stokes system, J. Funct. Anal., 262 (2012), 3556-3584.

[24] J. Simon, Nonhomogeneous viscous incompressible fluids: existence of velocity, density, and pressure, SIAM J. Math. Anal., 21 (1990), 1093-1117.

[25] P. Zhang and Q. Qiu, Propagation of higher-order regularities of the boundaries of 3-D vortex patches, Chinese Ann. Math. Ser. A, 18 (1997), 381-390.

(X. Liao) Academy of Mathematics \& Systems Science and Hua Loo-Keng Key Laboratory of Mathematics, The Chinese Academy of Sciences, ChinA

E-mail address: xian.liao@amss.ac.cn

(P. Zhang) Academy of Mathematics \& Systems Science and Hua Loo-Keng Key Laboratory of Mathematics, The Chinese Academy of Sciences, China

E-mail address: zp@amss.ac.cn 